University of South Florida

DIGITAL COMMONS

Digital Commons @ University of

@ UNIVERSITY OF SOUTH FLORIDA

South Florida

USF Tampa Graduate Theses and Dissertations

USF Graduate Theses and Dissertations

10-16-2009

\title{
A Data Link Layer In Support Of Swarming Of Autonomous Underwater Vehicles
}

Daladier Jabba Molinares

University of South Florida

Follow this and additional works at: https://digitalcommons.usf.edu/etd

Part of the American Studies Commons

\section{Scholar Commons Citation}

Jabba Molinares, Daladier, "A Data Link Layer In Support Of Swarming Of Autonomous Underwater Vehicles" (2009). USF Tampa Graduate Theses and Dissertations.

https://digitalcommons.usf.edu/etd/2026

This Dissertation is brought to you for free and open access by the USF Graduate Theses and Dissertations at Digital Commons @ University of South Florida. It has been accepted for inclusion in USF Tampa Graduate Theses and Dissertations by an authorized administrator of Digital Commons @ University of South Florida. For more information, please contact digitalcommons@usf.edu. 
A Data Link Layer In Support Of Swarming Of Autonomous Underwater Vehicles

by

Daladier Jabba Molinares

\begin{abstract}
A dissertation submitted in partial fulfillment
of the requirements for the degree of

Doctor of Philosophy

Department of Computer Science \& Engineering

College of Engineering

University of South Florida
\end{abstract}

Major Professor: Miguel A. Labrador, Ph.D.

Ken Christensen, Ph.D.

Wilfrido A. Moreno, Ph.D.

Rafael Perez, Ph.D.

William Stark, Ph.D.

Date of Approval:

October 16, 2009

Keywords: acoustic communications, mac sublayer, logical link control sublayer, multichannel communications, ofdma, hidden markov model

(C) Copyright 2009 , Daladier Jabba Molinares 
To my family 


\section{Acknowledgements}

I would like to acknowledge my advisor Dr. Miguel Labrador, for his direction, support, patience and considerable contributions to my research. The environment he has for his students, filled with freedom, resources and challenges, has created an ideal research space.

I would like to thank the University of South Florida, Universidad del Norte and LASPAUCOLCIENCIAS for supporting my studies. I would also like to sincerely thank the members of my committee Dr. Perez, Dr. Christensen, Dr. Stark, and Dr. Moreno for their valuable comments and suggestions.

I thank all my friends for their continuous encouragement and support; with these people I engaged in discussions, worked on projects and experiments, and received their help. Words are not enough to express my thanks to my family for their constant and unconditional support and love, but especially to Salome, my piece of heaven, and to my wife Milena, who encouraged me to fulfill this dream. She is everything to me.

Finally, I thank God for guiding my life and my dreams, without Him nothing would be possible. 


\section{Table of Contents}

List of Tables $\quad$ vii

List of Figures $\quad$ viii

Abstract $\quad$ xiii

Chapter 1 Introduction 1

1.1 Underwater Networks 1

1.1.1 Static Two-Dimensional Underwater Acoustic Networks for Ocean Bottom Monitoring 3

1.1.2 Static Three-Dimensional Underwater Acoustic Networks for Ocean-Column Monitoring 4

1.1.3 Three-Dimensional Networks of AUVs 5

1.1.4 AUV Networks 6

1.2 Underwater Communications and the Data Link Layer 8

1.3 MAC Sublayer in Underwater Ad Hoc Networks 12

1.4 Logical Link Control Sublayer in Underwater Ad Hoc Networks 16

$\begin{array}{lll}1.5 & \text { Contributions } & 16\end{array}$

$\begin{array}{ll}\text { 1.6 Organization of the Dissertation } & 18\end{array}$ 
2.2.3.1 A Networking Protocol for Underwater Networks [17]

2.2.3.2 Modified Media Access Control Design for the Acoustic-Based Underwater Digital Data Communication [18]

2.2.3.3 Adapted MACA to Underwater Acoustic Networks [19]

2.2.3.4 Slotted FAMA, Protocol for Underwater Acoustic Networks [21]

2.2.3.5 UWAN-MAN, an Energy-Efficient MAC Protocol for Underwater Acoustic Wireless Sensor Networks [22]

2.2.3.6 A Hybrid Medium Access Control Protocol for Underwater Wireless Networks [3]

2.2.3.7 T-Lohi, a New Class of MAC Protocols for Underwater Acoustic Sensor Networks [24]

2.2.3.8 A MAC Protocol for Underwater Sensor Networks [26] 
2.3.1.1 ARQ Protocols

2.3.1.2 Forward Error Correction Protocols

2.3.1.3 Hybrid Protocols

2.3.2 Underwater Logical Link Control Protocols

2.3.2.1 Optimization of a Data Link Protocol [35]

2.3.2.2 A Multi-Hop ARQ Protocol [37]

2.3.2.3 FEC-Based Reliable Data Transport Protocol for Underwater Sensor Networks [38]

Chapter 3 2MAC: A Multichannel MAC Protocol

3.1 2MAC Description

3.2 Network Topology and Channel Assignment

3.3 Scenario

3.4 2MAC State Transitions

3.4.1 Idle State

3.4.2 Channel Assignment State

3.4.3 Contention (Listen) State

3.4.4 Waiting for CTS State

3.4.5 Receiving RTS State

3.4.6 Waiting for ACK State

3.4.7 Waiting for Data State

3.4.8 Backoff State

3.4.9 Adjusted Response State 
Chapter 4 SW-MER: A Stop and Wait Window-Based Logical Link Control Protocol With Exponential Retransmissions

4.1 SW-MER Description

4.2 SW-MER State Transitions

4.2.1 Selecting Possible Packets

4.2.2 Updating Copies of Each Packet in the Window

4.2.3 Listen

4.2.4 Waiting for CTS

4.2.5 Sending Window of Packets

4.2.6 Waiting for ACK Vector

4.2.7 Identifying Packets Sent With Errors

4.2.8 Receiving RTS

4.2.9 Preparing the CTS to be Sent

4.2.10 Waiting to Receive Packets

4.2.11 Checking Packets

4.2.12 Enqueuing Packets without Errors

4.2.13 Verifying Packets to be Sent

4.2.14 Generating the ACK Vector

Chapter 5 Analytical Models for the Proposed Data Link Layer Protocols 
5.1.2 Throughput Analysis $\quad 83$

5.1.2.1 Packet Transmission Probability 83

5.1.2.2 Saturation Throughput 91

$\begin{array}{lll}\text { 5.1.3 Model Validation } & 98\end{array}$

$\begin{array}{lll}5.2 & \text { Analytical Model for SW-MER } & 100\end{array}$

5.2.1 SW-MER Transmission Process 100

$\begin{array}{lll}\text { 5.2.2 Throughput Analysis } & 102\end{array}$

$\begin{array}{lll}\text { 5.2.3 Model Validation } & 104\end{array}$

$\begin{array}{lll}\text { Chapter } 6 & \text { Performance Evaluation } & 107\end{array}$

$\begin{array}{lll}\text { 6.1 Scenarios and Parameters } & 107\end{array}$

$\begin{array}{ll}\text { 6.2 Channel Error Models } & 108\end{array}$

6.3 Performance Evaluation for 2MAC 110

6.4 Performance Evaluation for SW-MER 111

6.5 Performance Evaluation for the Entire Data Link 116

Chapter 7 An Adaptive Logical Link Sublayer Protocol in Response to Underwater Acoustic Communication (UAC) Channel Changes 120

$\begin{array}{lll}7.1 & \text { Adaptive SW-MER Description } & 121\end{array}$

$\begin{array}{ll}\text { 7.2 Determining the Channel Quality } & 121\end{array}$

7.3 States of the Adaptive Logical Link Protocol 125

$\begin{array}{lll}\text { 7.3.1 Listen } & 126\end{array}$

7.3.2 Verifying the State of the Channel 126 
7.3.3 Updating Channel Status History

7.3.4 Updating Copies of Packets to be Sent

7.3.5 Waiting to Receive Packets

7.3.6 Checking Packets

7.3.7 Enqueueing Packets Without Errors

7.3.8 Verifying Packets to be Sent to the Upper Layer

7.3.9 Generating the ACK Vector

7.3.10 Identifying Packets Sent With Errors

7.4 SW-MER Protocol Example

7.5 Channel Error Models

7.6 Performance Evaluation

7.6.1 Simulation Parameters

7.6.2 Throughput Evaluation

Chapter 8 Conclusions and Future Work

8.1 Conclusions

8.2 Future Work

List of References

Appendices

Appendix A: The Stationary Distribution of the Markov Chain

Appendix B: The Throughput Efficiency of SW-MER 


\section{List of Tables}

$\begin{array}{llr}\text { Table } 1.1 & \text { Node power consumption [3]. } & 9\end{array}$

Table 5.1 Notations used to obtain the saturation throughput. 93

Table 5.2 Parameter values used to validate the saturation throughput. 99

$\begin{array}{ll}\text { Table 5.3 Notations used to obtain the throughput efficiency. } & 101\end{array}$

Table 5.4 Parameter values used to validate the throughput efficiency. 105

Table 6.1 Parameter values used to evaluate 2MAC and SW-MER. 108

$\begin{array}{lll}\text { Table } 7.1 & \text { Simulation parameters. } & 133\end{array}$ 


\section{List of Figures}

Figure 1.1 Static two-dimensional underwater acoustic networks [1].

Figure 1.2 Static three-dimensional underwater acoustic networks [1].

Figure 1.3 Three-dimensional networks of autonomous underwater vehicles [1]. $\quad 6$

$\begin{array}{lll}\text { Figure 1.4 AUV networks. } & 7\end{array}$

$\begin{array}{lll}\text { Figure 1.5 Reflection in water. } & 10\end{array}$

$\begin{array}{lll}\text { Figure 1.6 Refraction of sound in water. } & 10\end{array}$

$\begin{array}{lll}\text { Figure } 1.7 \text { Hidden terminal problem. } & 13\end{array}$

$\begin{array}{lll}\text { Figure } 1.8 & \text { Exposed terminal problem. } & 13\end{array}$

$\begin{array}{lll}\text { Figure } 1.9 \text { Capture problem. } & 14\end{array}$

$\begin{array}{ll}\text { Figure 1.10 Deafness problem. } & 15\end{array}$

$\begin{array}{lll}\text { Figure } 2.1 & \text { OFDMA. } & 21\end{array}$

Figure 2.2 Comparison between OFDM and OFDMA. 21

Figure 2.3 Classification of MAC protocols based on the number of channels. 22

Figure 2.4 Classification of MAC protocols based on their network architecture.

Figure 2.5 Topology Discovery Message (TDM) Propagation in an underwater acoustic network [17]. 
Figure 2.6 Adaptation of MACA protocol to underwater acoustic communications [20].

Figure 2.7 Slotted FAMA [21].

Figure 2.8 Hybrid protocol frame structure [3].

Figure 2.9 A MAC protocol using RTS/CTS handshaking [26].

Figure 2.10 Classification of logic control protocols.

Figure 2.11 One-dimensional n-hop acoustic channel [37].

Figure 2.12 Two-dimensional multi-hop acoustic sensor network [37].

Figure 3.1 2MAC transmission process.

Figure 3.2 Linear topology.

Figure 3.3 Hexagon topology.

Figure 3.4 Nonagon topology.

Figure 3.5 Finite state machine of 2MAC process at the sender.

Figure 3.6 Finite state machine of $2 \mathrm{MAC}$ process at the receiver.

Figure 3.7 2MAC, transmission diagram.

Figure 3.8 Contention process.

$\begin{array}{lll}\text { Figure } 3.9 & \text { RTS/CTS communication process. } & 61\end{array}$

Figure 3.10 Different backoffs using packet size $=150$ bytes and $B E R=1 \times 10^{-3} . \quad 65$

Figure 3.11 Different backoffs using packet size $=300$ bytes and $B E R=1 \times 10^{-4} . \quad 65$

Figure 3.12 Throughput with different backoff algorithms.

Figure 4.1 First transmission, $M=6$ and packets 2 and 3 arrive with errors.

Figure 4.2 Second transmission, $M=6$ and packet 3 arrive with errors again. 
Figure 4.3 Finite state machine of the process in the sender at the LLC layer.

Figure 4.4 Finite state machine of the process in the receiver at the LLC layer.

Figure 5.1 Markov chain for the backoff process [46].

Figure 5.2 Transmission periods in an error-prone channel.

Figure 5.3 Throughput comparison with $B E R=1 \times 10^{-3}$.

Figure 5.4 Throughput comparison with $B E R=1 \times 10^{-4}$.

Figure 5.5 SW-MER, transmission process.

Figure 5.6 Throughput efficiency comparison with a window size of 8 packets.

Figure 5.7 Throughput efficiency comparison with a window size of 16 packets.

Figure 5.8 Throughput efficiency comparison with a window size of 32 packets.

Figure 6.1 Two-state Markov model representation.

Figure 6.2 Throughput of the MAC protocols, packet size of 1200 bits.

Figure 6.3 Throughput of the MAC protocols, packet size of 2400 bits.

Figure 6.4 Throughput efficiency for a window size of 8 data packets using the Bernoulli error model.

Figure 6.5 Throughput efficiency for a window size of 16 data packets using a Bernoulli error model.

Figure 6.6 Packet delivery rate for a window size of 8 data packets using the Bernoulli error model.

Figure 6.7 Packet delivery rate for a window size of 16 data packets using the Bernoulli error model.

Figure 6.8 Throughput efficiency for a window size of 16 packets using the shallow water error model. 
Figure 6.9 Packet delivery rate for a window size of 16 data packets using the shallow water error model.

Figure 6.10 Throughput of the combined SW-MER and 2MAC protocols using packet size of 1200 bits, and $\mathrm{BER}=1 \times 10^{-3}$.

Figure 6.11 Throughput of the combined SW-MER and 2MAC protocols using packet size of 1200 bits, and Markov model channel.

Figure 6.12 Throughput of the combined SW-MER and 2MAC protocols using packet size of 2400 bits.

Figure 7.1 Transmission between nodes located at different depths.

Figure 7.2 Four-state machine representation.

Figure 7.3 Physical and data link layer, interaction.

Figure 7.4 Finite state machine of the sender process.

Figure 7.5 Finite state machine of the receiver process.

Figure 7.6 Example of the proposed adaptive stop and wait sliding windowbased mechanism.

Figure 7.7 Linear topology.

Figure 7.8 Copies per packet using a Bernoulli error model, a window size of 8 packets, a packet size of 2400 bits, $B E R=1 \times 10^{-3}$, and data rate of 2400 bps.

Figure 7.9 Copies per packet using a Bernoulli error model, a window size of 8 packets, a packet size of 2400 bits, $B E R=1 \times 10^{-3}$, and data rate of 19600 bps.

Figure 7.10 Copies per packet using a shallow water error model, a window size of 8 packets, a packet size of 1200 bits, and data rate of $2400 \mathrm{bps}$.

Figure 7.11 Throughput using a Bernoulli error model, a window size of 8 data packets, a packet size of 2400 bits, $\mathrm{BER}=1 \times 10^{-3}$, and data rate of 2400 bps. 
Figure 7.12 Throughput using a Bernoulli error model, a window size of 8 data packets, a packet size of 2400 bits, BER $=1 \times 10^{-3}$, and data rate of 19600 bps.

Figure 7.13 Copies per packet using a shallow water error model, a window size of 16 packets, a packet size of 2400 bits, and data rate of $19600 \mathrm{bps}$.

Figure 7.14 Throughput using a Bernoulli error model, a window size of 16 data packets, a packet size of 2400 bits, $B E R=1 \times 10^{-3}$, and data rate of 19600 bps.

Figure 7.15 Throughput using a shallow water error model, a window size of 8 packets, and a packet size of 1200 bits.

Figure 7.16 Throughput using a shallow water error model, a window size of 8 packets, and a packet size of 2400 bits. 


\title{
A Data Link Layer in Support of Swarming of Autonomous Underwater Vehicles
}

\author{
Daladier Jabba Molinares
}

\begin{abstract}
Communication underwater is challenging because of the inherent characteristics of the media. First, common radio frequency (RF) signals utilized in wireless communications cannot be used under water. RF signals are attenuated in such as way that RF communication underwater is restricted to very few meters. As a result, acoustic-based communication is utilized for underwater communications; however, acoustic communication has its own limitations. For example, the speed of sound is five orders of magnitude lower than the speed of light, meaning that communications under water experience long propagation delays, even in short distances. Long propagation delays impose strong challenges in the design of Data Link Layer (DLL) protocols.
\end{abstract}

The underwater communication channel is noisy, too. The bit error rate (BER) can also change depending on depth and other factors, and the errors are correlated, like in wireless communications. As in wireless communications, transducers for acoustic communication are half duplex, limiting the application of well-known detection mechanisms in Medium Access Control (MAC) layer protocols. Further, known problems like the hidden and exposed terminal problem also occur here. All these aspects together make the underwater communication channel to have the worst characteristics of all other known 
channels. Because of these reasons, underwater scenarios are complicated to implement, especially when they have underwater autonomous vehicles exchanging information among them.

This dissertation proposes data link layer protocols in support of swarming of underwater autonomous vehicles that deal with the problems mentioned before. At the MAC sublayer, a MAC protocol called 2MAC is introduced. 2MAC improves the throughput of the network using the multichannel capabilities of OFDM at the physical layer. At the logical link control sublayer, a protocol named SW-MER is proposed. SW-MER improves the throughput and the reliability combining the well-known stop and wait protocol with the sliding window strategy, and using an exponential retransmission strategy to deal with errors. 2MAC and SW-MER are evaluated and compared with other protocols using analytical means and simulations.

The results show that by using 2MAC, packet collisions are considerably reduced and the throughput improved. In addition, the use of SW-MER improves the packet delivery ratio over existing mechanisms. In general, the evaluations indicate that the proposed data link layer protocols offer a better communication alternative for underwater autonomous vehicles (UAV) than traditional protocols. 


\section{Chapter 1: Introduction}

\subsection{Underwater Networks}

The ocean is an scenario that has started to generate lot of interest in the researchers. There are different situations undersea that can be explored, and it is necessary to have an adequate underwater network to collect the information that will be processed later for different purposes. Obtaining information underseas such as by exploration, tactical surveillance or data collection is necessary for disaster prevention, civilian applications or for military tactics, and underwater devices are the resources that must be used in order to collect the data. To do this, underwater networks must be deployed in the areas of the ocean which need to be examined. Some of the scenarios like exploration and data collection under water, may require autonomous vehicles moving, collecting and exchanging information among them. In order to support swarming of Autonomous Underwater Vehicles (AUVs), it is very important to have constant, and reliable communication among the AUVs. The problem is that communication under water is very challenging.

First, radio frequency (RF) signals utilized in wireless communications cannot be used underwater because electromagnetic waves do not propagate well in that medium. RF signals are attenuated in such way that RF communication underwater would be restricted to very few meters. As a result, acoustic has been used in underwater communications. 
However, acoustic communication has its own limitations as well. For example, the speed of sound is not constant under water; it varies with depth, salinity, and other factors. Also, the speed of sound is five orders of magnitude lower than the speed of light (radio), meaning that communications under water experience long propagation delays, even in short distances.

In addition, the underwater communication channel is noisy and the bit error rate (BER) also changes, depending on the depth and other factors. Normally, the errors are correlated, like in wireless communications. Similarly, the amount and type of errors require new data link layer protocols.

Finally, as in wireless communications, transducers for acoustic communication are half duplex, limiting the application of well-known detection mechanisms in MAC layer protocols. All these aspects together make the underwater communication channel the worst medium compared with other networks, and impose strong challenges in the design of Data Link Layer (DLL) protocols.

There are different applications in which underwater networks can be applied, and the architecture design that supports those networks can vary depending on the particular characteristics of the corresponding scenario. Some of the applications for underwater communications are the following:

- Environment monitoring

- Human activities affecting the marine ecosystem such as chemical analysis of industrial waste

- Undersea explorations

- Detection of underwater oilfields 
- Disaster prevention

- Monitoring of ocean currents and winds, for example tsunamis

- Assisted navigation

- Location of dangerous rocks in shallow waters

- Distributed tactical surveillance

- Intrusion detection

For the design of underwater communications, three accepted architectures in underwater communications are described in [1]. These architectures are: static two-dimensional underwater acoustic networks for ocean bottom monitoring, static three-dimensional underwater acoustic networks for ocean-column monitoring, and three-dimensional networks of AUVs. A fourth architecture in this dissertation is presented, similar to the third one in [1], and will be named as AUV networks. All these architectures are explained in the following sections.

\subsubsection{Static Two-Dimensional Underwater Acoustic Networks for Ocean Bottom Monitoring}

As shown in Figure 1.1, clusters are built by groups of nodes and one of the nodes in every cluster represents a cluster-head. Nodes in this network are anchored to the bottom of the ocean and they can communicate directly with the cluster-head or via multi-hop. There are two type of communications, horizontal and vertical. The horizontal is between the nodes in a cluster with their cluster-head. The vertical is the communication between 
the cluster-head of every cluster with the surface station. The cluster-head is called the underwater gateway. In this architecture the base station not only can be equipped with acoustic links but also with radio frequency $(\mathrm{RF})$ communications or satellite transmitter

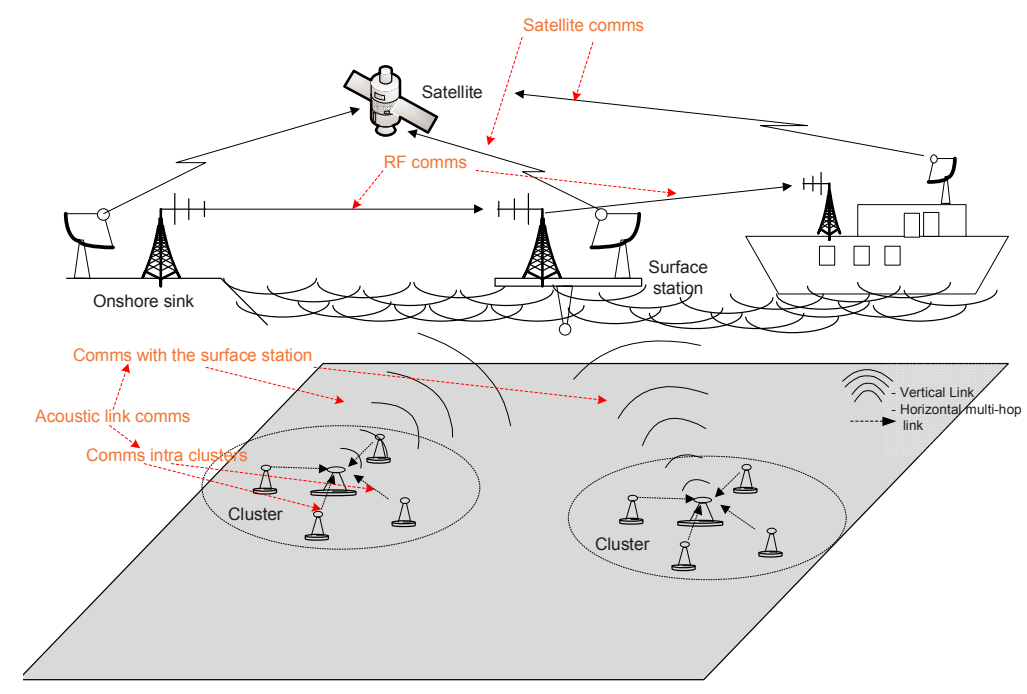

Figure 1.1: Static two-dimensional underwater acoustic networks [1].

to communicate with the onshore sink and/or the surface sink. There are some problems presented in this architecture, like the power necessary to transmit from nodes to the underwater gateway or from this to the surface station. Also multi-hop paths can increase both the complexity of the routing protocols and the signaling overhead over the network.

\subsubsection{Static Three-Dimensional Underwater Acoustic Networks for Ocean-Column Monitoring}

In these networks all nodes are static, and are used in case the scenario cannot be well modeled using sensors located at the bottom of the ocean. The principal difference from the first architecture is that this architecture does not build clusters and therefore there is only vertical communication, as shown in Figure 1.2. Here, nodes can be at different 


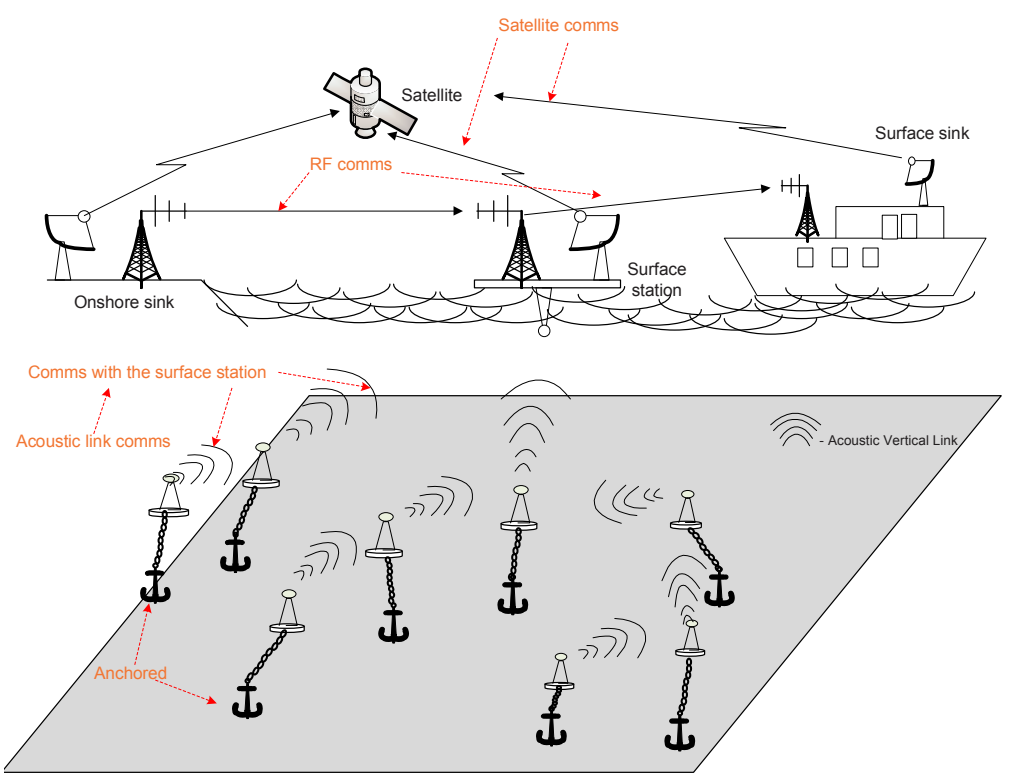

Figure 1.2: Static three-dimensional underwater acoustic networks [1].

depths (using floating buoys or with cables anchored to the bottom of the ocean) to collect information. Data is sent to the surface station directly or via multi hopping through the rest of the nodes. Some problems presented in this architecture are such that buoys are vulnerable to weather, can obstruct ships navigating on the surface, and they can be easily detected by the enemy if the scenario is for military purposes.

\subsubsection{Three-Dimensional Networks of AUVs}

As seen in Figure 1.3, nodes in these networks are also anchored to the bottom of the sea. They can be at different depths collecting information, but this information is stored until AUVs can collect it. AUVs are equipped with antennas, which requires network coordination among the vehicles. In this architecture is necessary to have algorithms that guarantee the coordination between the AUV and the sensors, and their correct functionality in the scenario. 


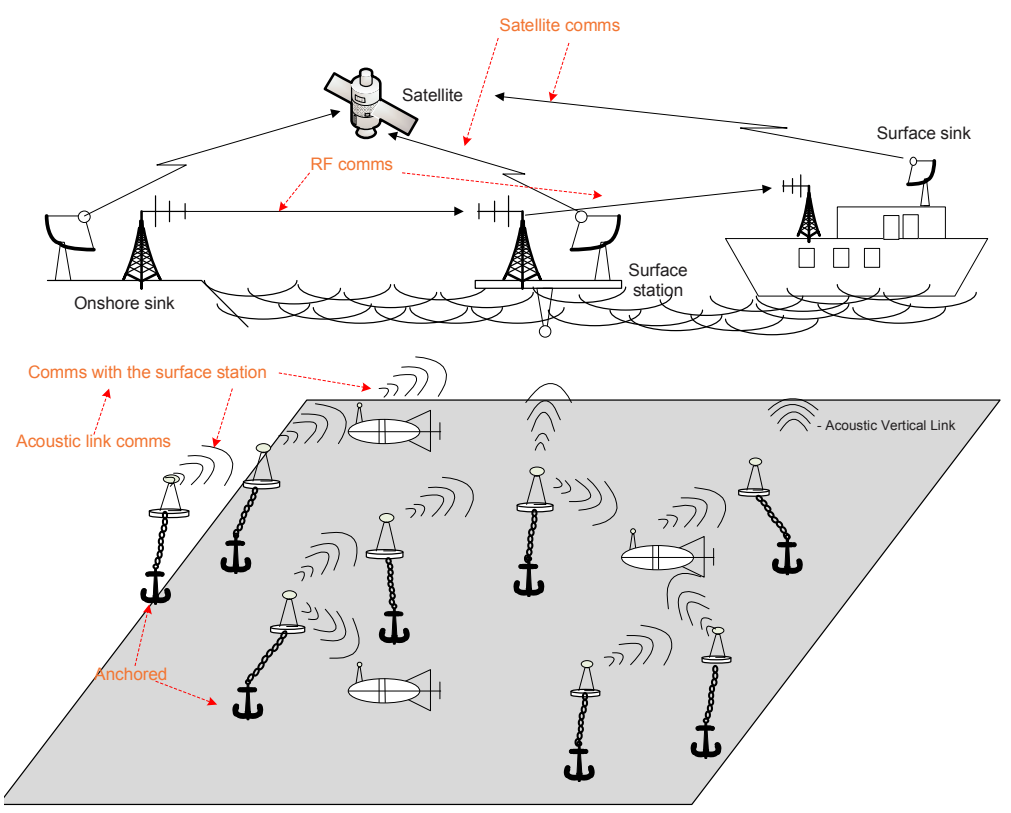

Figure 1.3: Three-dimensional networks of autonomous underwater vehicles [1].

\subsubsection{AUV Networks}

This is the most adequate architecture for the data link layer proposed in this dissertation. AUVs are equipped with communication systems to communicate with each other for swarming purposes, and sensors to collect the information required in scenarios such as detecting mine-like objects and oilfields, collecting water quality information and detecting intruders. Sensors either anchored to the ocean bottom or attached to buoys are not needed in this architecture, as seen in Figure 1.4. The application used in this dissertation, a swarm of AUVs for sea exploration, has the following description:

- Small number of AUVs (less than 10)

- AUV's speed is typically limited to 3-5 knots

- Depth of around $200 \mathrm{~m}$ 


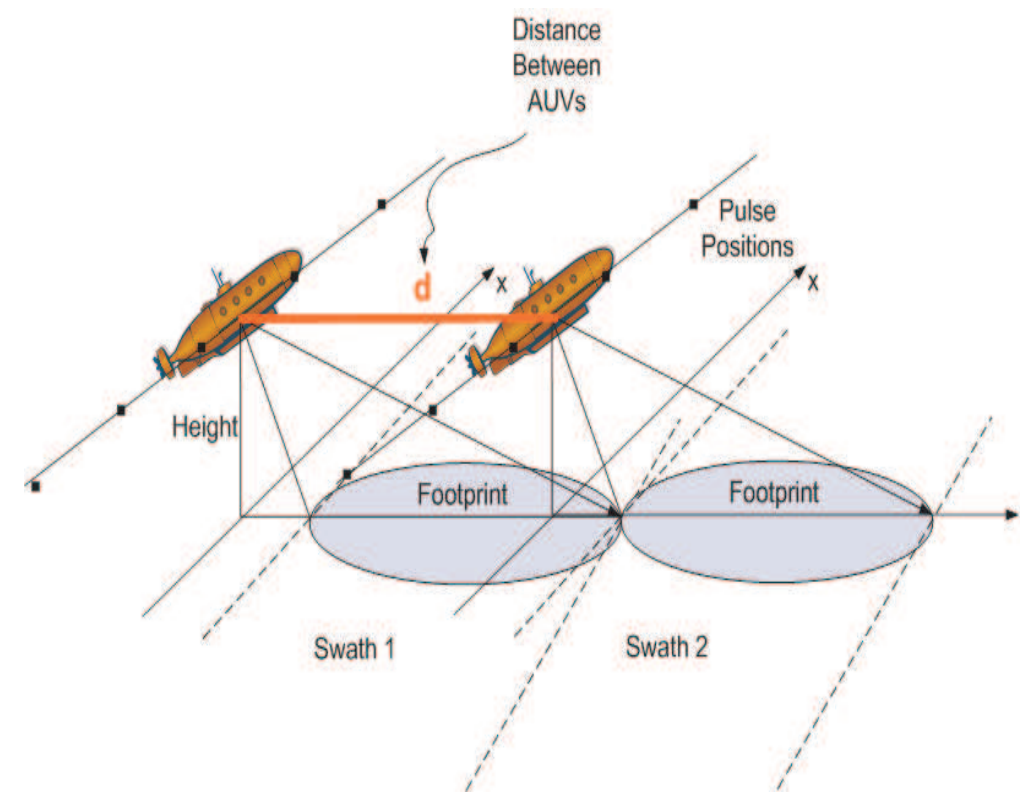

Figure 1.4: AUV networks.

- AUVs assume the existence of a localization system that they will use for autonomous navigation

- Low rate information exchange among the AUVs for telemetry, coordination, and planning

- A Synthetic Aperture Sonar (SAS) sensing and processing device is assumed

- SAS swath width of $250 \mathrm{~m}$ is assumed

- A maximum communication range of $1 \mathrm{~km}$ is assumed although the SAS swath width will determine the real value

For this application the continuous and reliable transmission of location data among AUVs for maintaining the formation is of great importance. For all these architectures, it is necessary to have reliable MAC and LLC protocols in order to establish adequate communication among the nodes in the network. It is important not only to save time at the 
moment of accessing the medium and deciding which node is going to start sending data, but also to reduce the number of collisions at the moment of sending packets, and packet loss due to the bit error rate, which is high in underwater networks. Further, new mechanisms are needed to improve the throughput due to long propagation delays.

The data link layer proposed in this dissertation addresses these problems. First, a new multichannel MAC sublayer protocol tailored to work with the Orthogonal FrequencyDivision Multiple Access (OFDMA) technology is introduced. The proposed MAC protocol reduces collisions and improves the throughput of the network. Second, a new LLC protocol is proposed to improve the reliability and the throughput of the transmission. In general, the goals of the new data link protocols are to reduce packet collisions, improve the throughput, achieve better channel utilization, and increase the packet delivery ratio.

\subsection{Underwater Communications and the Data Link Layer}

The data link layer is responsible for grouping the bits from the physical layer into logical chunks of data called frames, providing the means to transfer data between two adjacent users in a network, and detecting and correcting possible errors that could occur in the physical layer. This layer is split into two sublayers and each one has its own characteristics and tasks to execute. The sublayers are the MAC layer and the LLC layer.

The following are important issues to consider in the design of an underwater data link layer:

- Half duplex radios. Since nodes cannot transmit and receive at the same time, stop and wait protocols are the most commonly implemented LLC protocols. 
- Burst channel errors. In underwater communications errors are correlated [2], meaning that they occur in bursts.

- Energy consumption. Energy consumption is a critical issue not only in wireless but also in underwater communication systems, since underwater transceivers have large power ratios, and batteries are not easily replaceable as on land. Table 1.1 compares the power consumption of three devices: a modem for underwater communications (WHOI), RF sensor (Mica 2), and RF wireless computer networks (Cisco Aironet).

Table 1.1: Node power consumption [3].

\begin{tabular}{|c|c|c|r|}
\hline State & Underwater & RF Sensor & RF Computer \\
\hline $\mathrm{Tx}$ & $50 \mathrm{~W}$ & $80 \mathrm{~mW}$ & $2.24 \mathrm{~W}$ \\
\hline $\mathrm{Rx}$ & $3 \mathrm{~W}$ & $30 \mathrm{~mW}$ & $1.35 \mathrm{~W}$ \\
\hline Idle & $80 \mathrm{~mW}$ & $30 \mathrm{~mW}$ & $1.35 \mathrm{~W}$ \\
\hline
\end{tabular}

- Temporal and spatial fluctuation of frequency (space time uncertainty). Signal varies according to the channel geometry (spatial) and in time (temporal) in underwater communications. Due to the high latency medium (slow propagation in acoustic channels), collisions happen not only with concurrent transmissions, but also with transmissions at different time and distance.

- Propagation delays. Large propagation delays reduce the network throughput and channel utilization, and it is even worse when the exchange of several control packets is required to establish the communication between nodes in a network. This imposes important challenges in the design of efficient data link layer protocols.

- Long term fading. Ocean structures such as reefs and mountains, large ships, offshore underwater oil drilling equipment and internal waves over a long period of time, generate long term fading in water [4]. 


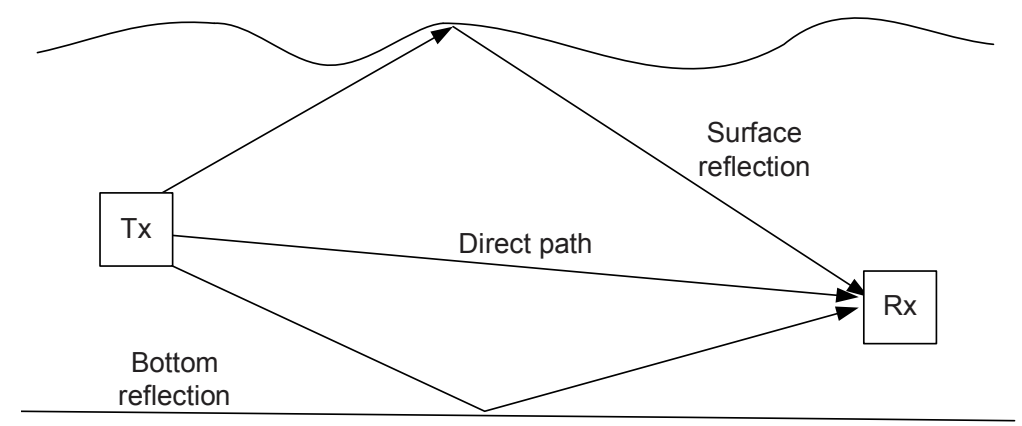

Figure 1.5: Reflection in water.

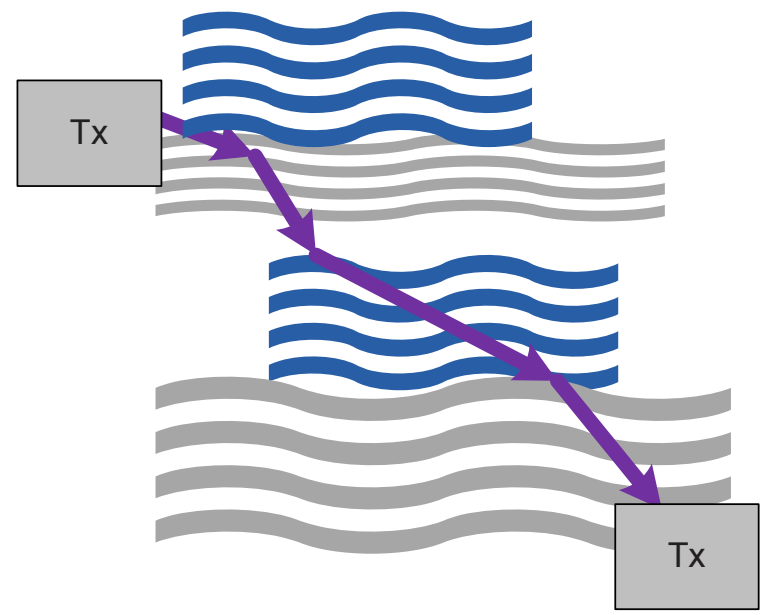

Figure 1.6: Refraction of sound in water.

- Short term fading because of multi path and delay spread. In multi path propagation and delay spread, the received signal power changes as a function of time because of reflection, diffraction, and scattering, causing signal fading for short periods of time. In underwater communications, multi path formation depends not only on the reflection on the surface (either bottom, on the water surface, or on any objects in between), but also on a direct path between transmitter and receiver, and on refraction or bending of rays depending on the depth, pressure and temperature of water at different water levels [4]. Figures 1.5 and 1.6 represent the reflections of rays and the refraction scenarios in water. 
- Short term fading because of time selectivity and Doppler spread. This short term fading gives random signal fluctuation or time variation on each channel response. This time variability is caused by the scattering on the waves of surface water in shallow water situations and scattering on deep sea waves in deep water. In addition, the motion of the transducer also contributes to Doppler spread [5]. On the other hand, because of the way rays arrive (rays arrive at different angles), different Doppler shifts appear, also creating short term fading.

- Inter Symbol Interference (ISI). This is totally different from noise; it is signal distortion in which one symbol interferes with subsequent symbols. This is an undesired phenomenon, as the previous symbols have a similar effect as noise, thus making the communication less reliable. ISI is usually caused by multipath propagation and the inherent non-linear frequency response of a channel [6].

- Synchronization. Achieving synchronization in underwater communications is challenging because of the long propagation delays.

- Bandwidth availability. Bandwidth is limited in underwater communications compared with wireless. Even though there is a long range system operating over several tens of kilometers, this is limited to a few $\mathrm{kHz}$ of bandwidth [4]. For that reason, MAC protocols need to be efficient.

- Path loss. Path loss represents the attenuation of the propagated information signal over distance. This attenuation in water for high frequency radio, especially in electrically more conductive salt water, is extremely high. If it is assumed that an average conductivity from seawater is 4 mhos/meters, and 0.05 mhos/meters in fresh water (tap water), as a consequence the attenuation for $2.4 \mathrm{GHz}$ will be around $1695 \mathrm{~dB} /$ meters in seawater, and $189 \mathrm{~dB} /$ meters in freshwater [4]. In underwa- 
ter communications, RF signals are more attenuated than in other media, making acoustic communication transmission the method of choice.

\subsection{MAC Sublayer in Underwater Ad Hoc Networks}

MAC protocols work as an interface between the LLC sublayer and the physical layer. They provide a physical address called the MAC address, and an addressing mechanism that is a unique serial number for every node in the network, allowing successful delivery of packets in a shared network. They also provide channel access control mechanisms required for sharing the common channel in the network (multiple access protocol). In general, MAC protocols regulate the access of a number of nodes to a shared medium, and are in charge of providing fair access to all the users sharing a common medium while achieving efficient channel utilization. A MAC protocol controls when a node can send a packet to a node (via unicast) or to a set of nodes (via multicast or broadcast).

Depending on the type of network, technology utilized, network topology, application requirements, and other factors, data link layer protocols have to deal with aspects such as Quality of Service (QoS) support, energy efficiency, synchronization, and error control (detection and recovery). Also, there are system constraints that are important to consider in the design of MAC protocols, such as the half duplex nature of the transceivers, underwater localization of the nodes and the available bandwidth.

The most important issues to solve in order to design underwater MAC protocols are the following:

- Hidden terminal problem. The hidden terminal problem occurs when a node is within the range of the intended destination but out of range of the sender. In Figure 


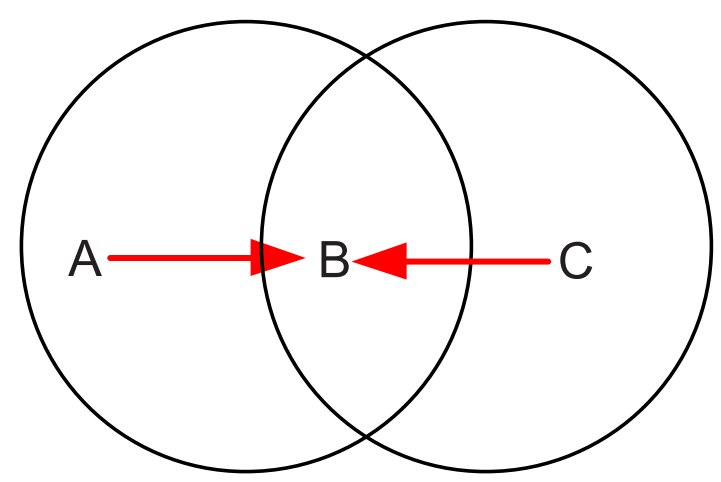

Figure 1.7: Hidden terminal problem.

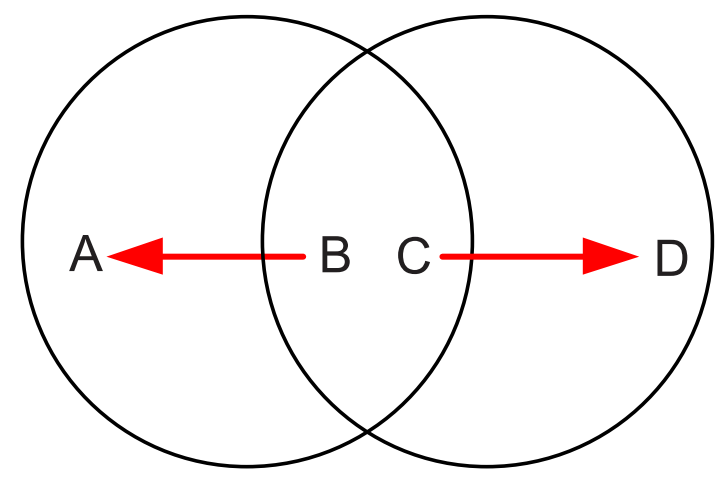

Figure 1.8: Exposed terminal problem.

1.7, node $\mathrm{A}$ transmits to $\mathrm{B}$, node $\mathrm{C}$ cannot hear $\mathrm{A}$, and also transmits to $\mathrm{B}$; as a result, there is a collision at $\mathrm{B}$. The hidden terminal problem can be substantially reduced by using an RTS/CTS handshake.

- Exposed terminal problem. The exposed terminal problem occurs when a node is prevented from sending packets to other nodes because a neighbor is already transmitting. In Figure 1.8, node $\mathrm{B}$ is transmitting to node $\mathrm{A}$ and node $\mathrm{C}$ has a packet to transmit to $\mathrm{D}$; as a consequence, $\mathrm{C}$ cannot transmit the data, although node $\mathrm{D}$ can receive the transmission without interference because it is out of range of $\mathrm{B}$. The exposed problem can be solved by separating control packets and data packets in different channels. 


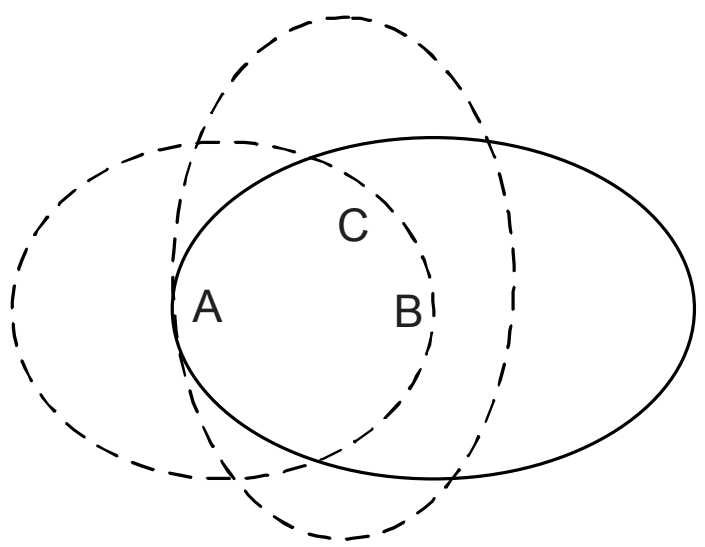

Figure 1.9: Capture problem.

- Synchronization. Some distributed MAC protocols need a synchronization mechanism. These protocols do not have a central node to control whether the channel is already busy or not, which node is using that channel, and for how long the channel will be used. Also it is necessary to have a synchronization mechanism to control when all the nodes in the network go to the sleeping mode to save energy.

- Capture problem. A capture problem is when two nodes send packets simultaneously to the same receiver and this node cleanly receives the information from one of them, such as in Figure 1.9. Nodes $\mathrm{A}$ and $\mathrm{C}$ send packets at the same time to node $\mathrm{B}$ but because the signal strength from $\mathrm{C}$ is higher than $\mathrm{A}$ at $\mathrm{B}, \mathrm{C}$ packets will be decoded without errors [7]. This situation can create unfair sharing of bandwidth, since $\mathrm{C}$ will have the channel all the time.

- Deafness problem. Another problem occurs when a node tries to transmit packets but the intended receiver is currently transmitting in an inactive channel of a third node (directional antennas are used), and the sender has to wait until the receiver is available; this problem is called the deafness problem, as seen in Figure 1.10. In this figure, there is a network with at least three nodes A, B, C; B starts 


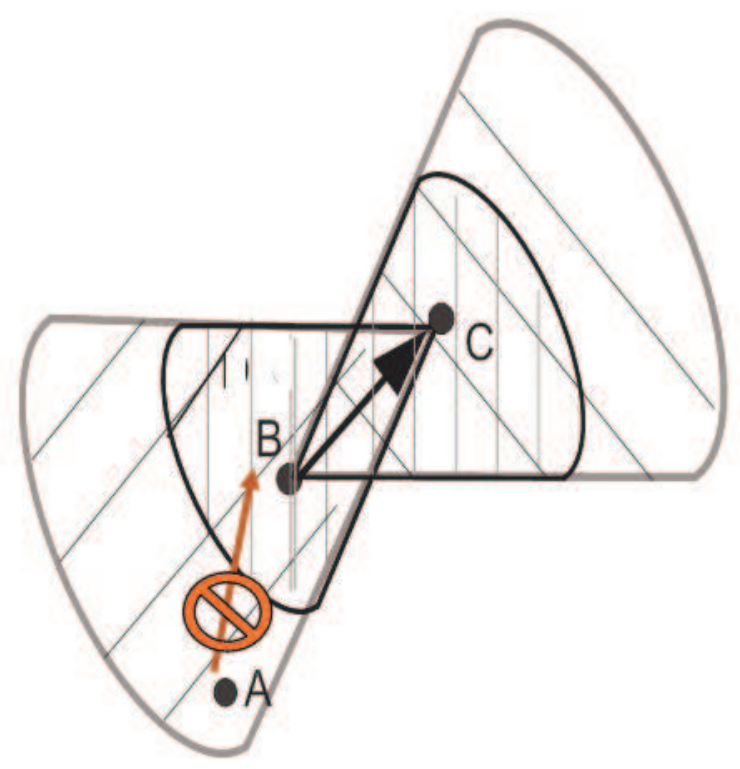

Figure 1.10: Deafness problem.

to send DATA to $\mathrm{C}$ after sending RTS and CTS control packets to each other. A is not aware of communication between $\mathrm{B}$ and $\mathrm{C}$ and attempts to send an RTS packet control to node B even though node B is deaf to node A.

As mentioned, due to the characteristics and the complications presented in the medium, the MAC sublayer in underwater communications is a critical protocol that must assure communication among the nodes in an effective and optimal way, so as to reduce collisions, waste of time and too much energy consumption. These problems start to increase when the number of nodes that communicate with each other at the same time is increased. In the literature some solutions are presented using not only one but multichannels with one or multiple antennas. The MAC protocol proposed in this dissertation provides an efficient solution that can be scalable to several nodes, having formations either linear or polygonal, without generating more collisions when nodes communicate with each other at the same time. 


\subsection{Logical Link Control Sublayer in Underwater Ad Hoc Networks}

The LLC is a sublayer that belongs to the data link layer in the OSI model. The underwater acoustic medium, like wireless network, is a half-duplex communication medium. For this reason, most of the protocols designed for underwater communications are based on modifications to the well-known stop and wait Automatic Repeat-ReQuest (ARQ) scheme. The LLC has the following functions:

- Implements flow control, using either the well-known stop and wait protocol, or the Sliding Window strategy.

- Implements error control.

- Ensures that data is transferred correctly between adjacent nodes in the underwater network.

The most important issues in this layer are to provide a reliable delivery of the packets, flow control, error detection and correction, better throughput and channel utilization [8]. The logical link protocol proposed in this dissertation uses exponential packet retransmissions guaranteeing a more reliable communication for the underwater communication, and combines the stop and wait scheme with a window packet transmission to improve the throughput and channel utilization.

\subsection{Contributions}

The primary research contribution of this dissertation is a new data link layer in support of swarming of autonomous underwater vehicles. This new data link layer is divided into 
two new protocols working together, one at the MAC sublayer and the other one at the LLC sublayer. The contributions in each layer are:

- A multichannel MAC layer protocol called 2MAC, tailored to work with OFDMA technology at the physical layer, is designed to reduce the hidden terminal problem, exposed terminal problem, capture problem, deafness problem and fairness, and improve the throughput of the underwater channel. With this protocol, the number of nodes in the network can be increased without increasing the number of collisions. This 2MAC protocol is presented in [9].

- A stop and wait, window-based LLC protocol called SW-MER is designed to improve the throughput in underwater channels. Also, SW-MER includes an exponential retransmission strategy to improve the packet delivery ratio. The SW-MER protocol is described in [9].

Other contributions are:

- A new backoff algorithm at the MAC layer that is more adequate for underwater communications. The proposed backoff algorithm is introduced in [9].

- An analytical model to calculate the saturation throughput of 2MAC.

- An analytical model to calculate the throughput efficiency of SW-MER.

- An improvement of SW-MER that adapts its transmission window in response to underwater acoustic communication channel changes. The adaptive SW-MER is introduced in [10].

- An underwater channel error model generated from synthetic traces that correspond to moving nodes in shallow water [9]. 


\subsection{Organization of the Dissertation}

The remainder of the dissertation is organized as follows. Chapter 2 reviews the existing literature in data link layer protocols for underwater acoustic communications. Chapter 3 presents the new MAC protocol. Chapter 4 describes the new stop and wait, windowbased protocol for the LLC layer. Chapter 5 presents the analytical model for each of the proposed data link protocols and a comparison between the analytical and experimental evaluations. Chapter 7 introduces an improvement of the proposed logical link protocol, by adapting the transmission window to different channel conditions. Details of the scenarios used for the proposed data link protocols, the adaptive protocol version, and their performance evaluations using some comparisons with other traditional data link layer

protocols, are presented in chapter 6 . Finally, chapter 8 summarizes the dissertation and presents direction for future research. 


\section{Chapter 2: Literature Review}

In this chapter, the OFDMA scheme is introduced, and the purpose of having OFDMA at the physical layer is explained. Later, an overview of MAC protocols for underwater communications is presented including the taxonomy and a brief description of the most important protocols in each category. Similarly, the next section presents LLC protocols for underwater communications, including the classification and some of the most important protocols available in the literature.

\subsection{Orthogonal Frequency-Division Multiple Access (OFDMA)}

There are issues in underwater MAC protocols such as the hidden and exposed terminal, capture, and deafness problem, that when combined with the characteristics of the channel make the performance of acoustic communications very poor. These problems can be solved to improve performance efficiency in terms of throughput. In [11] it is shown what is necessary to get these improvements and some results that have been achieved.

On the other hand, in systems where only one carrier is used, a single fade will cause the link to fail, but having a multicarrier system only some subcarriers will be affected. Orthogonal Frequency Division Multiplexing (OFDM) is a multicarrier transmission in which a single datastream is transmitted through several lower rate subcarriers. OFDM 
splits or multiplexes the radio signal into several small sub-signals that are transmitted at the same time at different frequencies to the receiver. The data carried is divided into several parallel data streams, one for each subcarrier, where each subcarrier is modulated with a conventional modulation scheme at a low symbol or bit rate. OFDM increases the efficiency of data communications by increasing the data throughput since there are several spaced subcarriers modulated.

Systems such as those that use OFDM are good candidates for underwater communications in which high data rate is involved with the existence of high delay spread channels. They can provide the same or even better performance efficiency, but offer a lowercomplexity implementation than underwater MAC protocols without using OFDM. Several papers on this topic have been written, such as [12], [13] and [14].

OFDM offers some advantages, like high frequency band efficiency, performance improvement for multipath interference, reduction of the selective fading anomaly, and the increase of system capacity [15] [16]. OFDM allows only one user at a time to use the channel, as seen in Figure 2.2. That means it is not possible to have several users at the same time using the medium. To have several users accessing the channel simultaneously, several transducers would have to be installed in each node in the network, and the hardware infrastructure can be costly. On the other hand, traditional MAC protocols are not adjusted to take advantage of using more than one channel simultaneously.

OFDMA is an extension of OFDM. It is a multi-user OFDM scheme that permits multiple access on the same channel. OFDMA uses multiple and closely spaced subcarriers, as seen in Figure 2.1, but the difference is that carriers in OFDMA are divided into groups of subcarriers. The subcarriers created are distributed by OFDMA among the users in such a way that users will transmit and receive at the same time using just one 


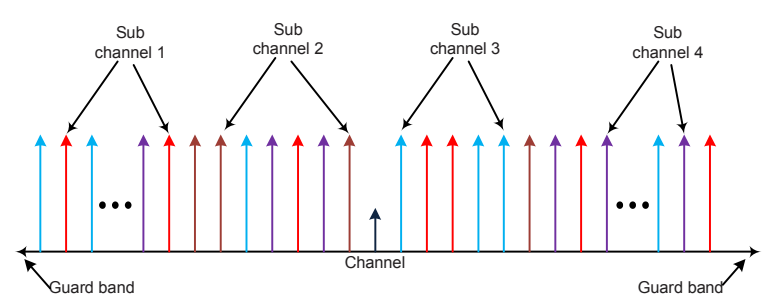

Figure 2.1: OFDMA.
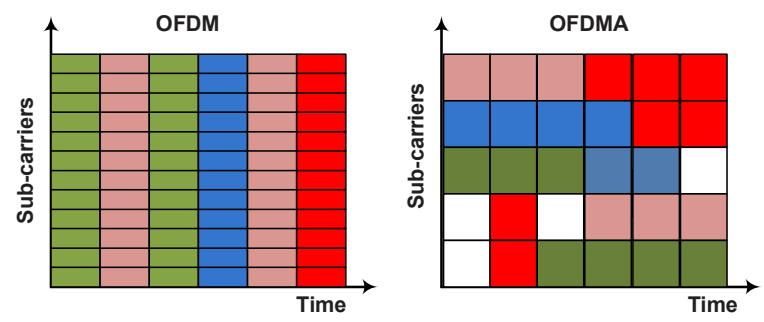

Figure 2.2: Comparison between OFDM and OFDMA.

channel, with having only one transceiver in each node (the cost in terms of hardware is not increased). Each user can have a group of subchannels, reducing problems like fading and interference presented in the network. Using an adequate topology and data link protocol in the data link layer with OFDMA, every node can transmit simultaneous data to different neighbors, eliminating the problems in underwater communications and increasing the throughput in the network. The cost in terms of hardware for supporting the proposed data link protocol is equivalent to having a standard underwater network, since requirements in terms of hardware are minimal, needing only a single half-duplex transceiver per node.

\subsection{Medium Access Control (MAC) Layer}

MAC protocols can be classified depending on the number of channels used: one channel and more than one, as shown in Figure 2.3, or their network architecture, as shown in 


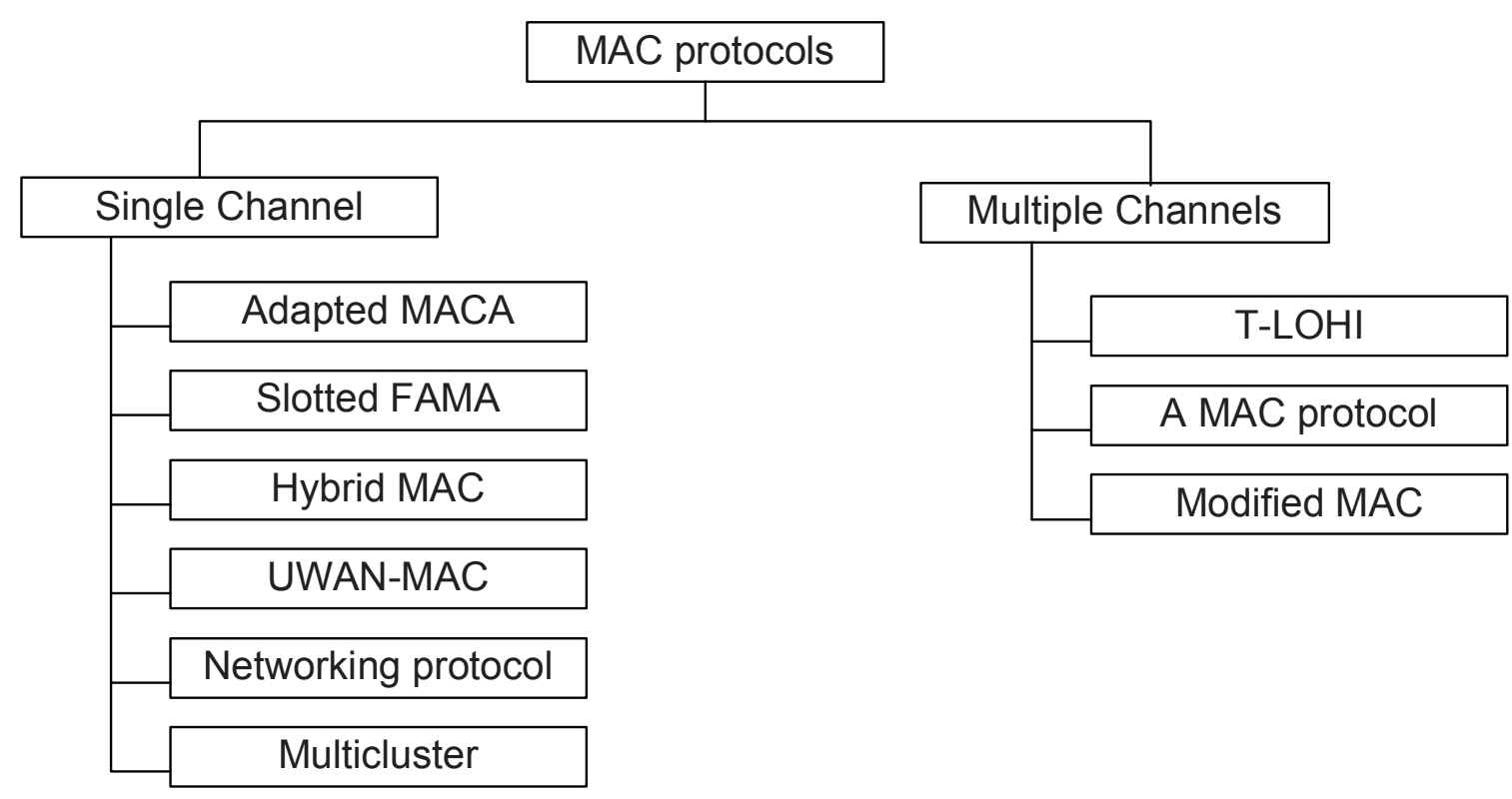

Figure 2.3: Classification of MAC protocols based on the number of channels.

Figure 2.4. These will be explained in the following sections. Some of the underwater MAC protocols that have been designed are displayed in the corresponding classification and described later.

\subsubsection{Channel Classification}

These protocols offer some advantages, such as multiple frequency channels that improve the capacity of an underwater network, the reduction or elimination of collisions, and the reduction of the hidden and exposed terminal problems. They may use more than one channel but only one transceiver or multiple channels with multiple transceivers, where the number of channels and transceivers does not necessarily match. As seen in Figure 2.3, protocols are divided into one channel or more than one channel used. In the first category, a channel is selected from the beginning on which nodes will communicate with 




Figure 2.4: Classification of MAC protocols based on their network architecture.

each other. In the second category, also called multi-channel protocols, every node can have not only more than one channel but also several transceivers. Two nodes could be transferring information through a specific interface and channel, and the other two nodes could be using either the same channel but a different transducer or the same transducer but a different channel.

There are some issues that are important to consider in the design of multi-channel MAC layer protocols for underwater networks. For example, when nodes try to communicate with each other through a specific channel, taking into account that there are several channels (some of them available) and there is only one NIC, nodes cannot know which channels are busy for other nodes in real time and they can create interference with other nodes; this problem is called the hidden multi-channel problem. How channels can be assigned to every node in the network is a complex problem, especially if there is just one NIC in each of them, since every NIC only can use one channel. 
In networks with a number of nodes it is difficult to determine when transducers are in sleeping or waking mode and also to establish the communication between nodes. Synchronization among the nodes in the network using either several channels or more than one NIC at each node is also a complex process and time-consuming. Another problem occurs when a node tries to transmit packets but the intended receiver is currently transmitting in an inactive channel of a third node. In this case, the sender has to wait until the receiver is available. This problem is called a Deafness problem.

\subsubsection{Network Architecture}

In this category, protocols can be classified into centralized, distributed and hybrid protocols. Centralized MAC protocols assume the existence of a base station in the network architecture. The base station has the responsibility of being the arbiter assigning channel resources to users according to a specific algorithm. As such, users are told when to transmit and receive information, for how long, at what rate, at what power, etc. All these calculations are performed by the base station, which is assumed to be a powerful device in terms of computation capabilities, energy resources, memory, etc.

Centralized protocols have advantages and disadvantages. Among the most important advantages, centralized MAC protocols allow for simple users, and there are no hidden and exposed terminal problems. On the down side, centralized mechanisms usually cannot scale to a large number of users, need a central and powerful station, and may present a single point of failure. Some of the most important centralized MAC protocols are shown in Figure 2.4. In distributed MAC protocols, stations make decisions about when to acquire the channel, i.e. stations contend for the channel. Because stations do not know 
when other stations acquire the channel, collisions are possible. Indeed, an important component of distributed algorithms is a collision detection and resolution algorithm. Distributed schemes are scalable compared with centralized mechanisms and do not require any special station to make decisions on behalf of the rest. Therefore, no single point of failure exists, and reliability is improved. However, distributed schemes must deal with collisions, as explained before, and also with the hidden and exposed terminal problems.

Hybrid protocols are a combination of centralized and distributed MAC protocols. Most of the hybrid protocols have been designed based on request-grant mechanisms. Before starting to transmit, nodes have to send a request for permission (channel reservation) to the base station telling how much bandwidth they need and for how long. The base station utilizes different algorithms to assign each user a portion of the channel. Contentionbased protocols such as ALOHA are normally utilized for the reservation phase.

\subsubsection{Underwater MAC Protocols}

This section provides an overview of the most relevant MAC layer protocols for underwater communications.

\subsubsection{A Networking Protocol for Underwater Networks [17]}

In this protocol, the authors focus on two objectives. The first goal is to reduce message latency by removing dependencies between data and control communications. The second one is to use a proactive approach for achieving a robust and efficient topology mainte- 


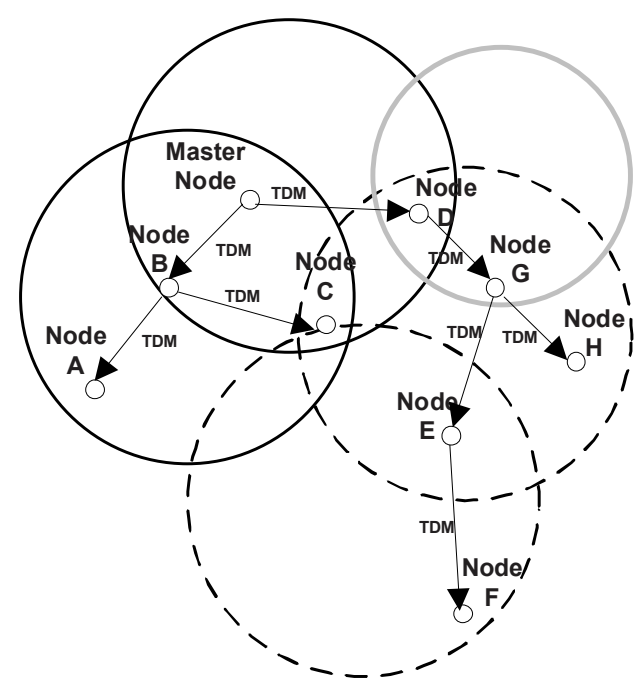

Figure 2.5: Topology Discovery Message (TDM) Propagation in an underwater acoustic network [17].

nance and routing. This process is done by a centric gateway that works as a master node. There is a proactive algorithm that helps the master node not only for creating and maintaining a tree topology for the network, but also for updating control information such as power level and routes. In addition, the protocol reduces the expected delay generated in the network by separating the control overhead from the data delivery.

The process starts with a network configuration cycle generated by the master node transmitting Topology Discovery Messages (TDM), called a configuration probe, and it is done via dedicated channels (dedicated channels are set by applying CDMA codes). Every node in the network will select a channel and send a response to the master node. As a consequence, the master node will know which channel has been selected for every node in its network, as shown in Figure 2.5. Also, information about topology is processed in each node (topology discovery) and sent when responding. This topology discovery will be done periodically. Once the topology has been created, routing paths are created by a proactive approach taking into account the existence of a master node (centralized 
routing). Due to the routing process through the master, there is a global planning of the resources in the network. For example, if one node has low battery power available, the master node will update the route traffic and use another path for transmissions. There are two type of paths in the network: paths that end at the master node and paths between non master nodes. The first type of path is used for routing traffic and the second for exchanging information between non-master nodes.

By using CDMA, each node that has assigned a channel for transmitting packets also must be able to listen to the rest of the channels that are being used for other nodes in the network. Having the control of the transmission range of each node in the master node will optimize the node power reserves and permit channel reutilization. On the other hand, creating dedicated transmission channels for each node will reduce the propagation delay problem.

One of the disadvantages of this protocol is that the capture problem, also called the nearfar problem, is presented in which the unfair sharing of bandwidth has not been well solved in acoustic networks. Another problem is that this protocol is centralized, based on a master node, and this is not the best option when mobile networks are implemented.

\subsubsection{Modified Media Access Control Design for the Acoustic-Based Underwater Digital Data Communication [18]}

This protocol works with multiple channels and a timer for each of them in every node in the network to transmit data packets. Each node just can use one channel to start a transmission with the receiver. The process is divided into four stages: carrier sensing, transmission frame, receiving frame and error control. In the carrier sensing stage, if 
carrier sensing is detected the flags for that channel are set to '0' and timer starts again, otherwise when overflow occurs due to the increasing idle time for each channel, the flag is increased and also the timer starts again. The transmission frame stage occurs by requesting a transmission signal from the sender, and one channel among them is selected for the process. A procedure for searching the candidate channels is executed and as a result, the channel with the longest idle time is selected for the transmission.

Once the frame arrives to the receiver, it will send either an ACK signal due to a successful transmission, or a NACK signal (Negative ACK due to frame error detection), and go to the carrier sensing mode. When the NACK is sent, the receiving frame stage is canceled. The error control stage is verified by using a stop and wait ARQ protocol in which an ACK packet indicates to the sender that the data packet arrived without errors to the receiver.

Some of disadvantages in this protocol are that the number of channels used by every node for data transmission and reception affects its connectivity in the network, generating as a consequence problems in terms of throughput; having local information about the state of the channels in each node is required to make decisions; and a scheduling process for sending data packets through the channels must be also defined.

\subsubsection{Adapted MACA to Underwater Acoustic Networks [19]}

A modified version of the original MACA protocol is proposed in which three type of packets are utilized: RTS (Request to Send) and CTS (Clear to Send) control packets to establish a connection between two nodes in the network, and DATA packets. The proposed protocol has been designed to work in an underwater acoustic sensor network 


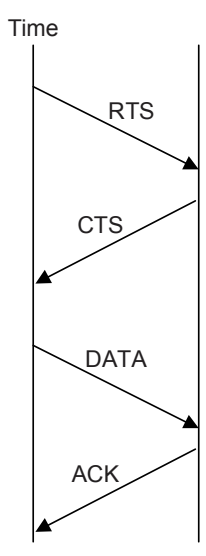

(a)

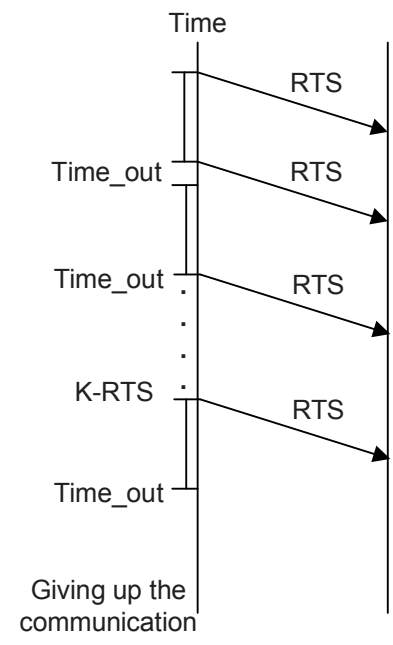

(b)

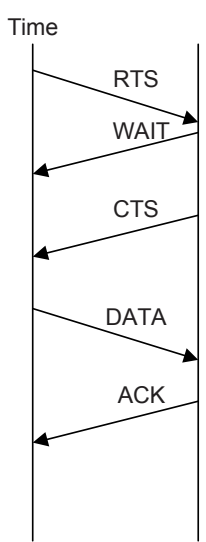

(c)

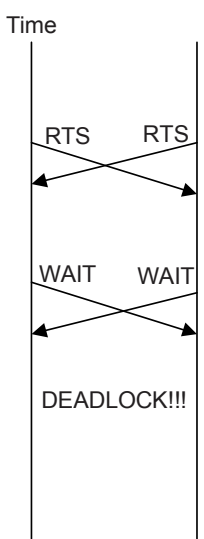

(d)

Figure 2.6: Adaptation of MACA protocol to underwater acoustic communications [20].

that includes a master node. Nodes are connected with the master one in a hierarchical manner, and the master node is in charge of sending the information to a surface buoy. Nodes start in a low-power state until they decide to transmit data packets, in which they will send an RTS packet.

Once the transmitter receives a CTS packet, it starts to send the DATA packet to the receiver, and later an ACK packet will be sent from the receiver as shown in Figure 2.6(a). If the sender has not received the CTS during a certain period of time, it will retransmit the RTS packet, repeating the process for a maximum number of $\mathrm{K}$ retransmissions as shown in Figure 2.6(b). After that, the source will decide that the link is not longer available and will go back to a low-power state.

During the time that the receiver sends the CTS, the sender starts to transmit the DATA packet and later receives the ACK, all other requests from the rest of the nodes to either the transmitter or receiver will be declined. The problem happens when the source whose transmission has been declined is not informed; it will be repeating its request periodi- 
cally, generating as a consequence a lot of unnecessary power consumption in the source and also an increase of the possibility of collisions. To avoid this, a WAIT command is added to inform the source that the destination is busy and will send the CTS as soon as possible. This is shown in Figure 2.6(c).

A deadlock problem appears with the utilization of the WAIT command. If two nodes send an RTS at the same time to each other, they will send later a WAIT command and wait forever for the other node to send a CTS, as in Figure 2.6(d). The problem is solved by assigning priority to the packets that are directed to the master node [19].

Some problems still happen in this protocol. Although the control packet exchanges are reduced, large packet collisions can still be present in dense networks. Problems like scalability will increase the delay because several nodes will try to access the network at the same time with having one channel, and only two of them can start the communication; the rest of the nodes will be waiting until the transmission finishes (the unfair sharing of bandwidth).

\subsubsection{Slotted FAMA, Protocol for Underwater Acoustic Networks [21]}

This is based on the floor acquisition multiple access (FAMA) protocol which combines carrier sensing and an exchanging of control packets between sender and receiver before data transmission starts. This protocol uses time slots to control packets from consuming excessive time in their transmission, which is a problem in underwater acoustic communications. Two problems that are important to deal with in underwater communications are low bandwidth and long propagation delay (caused by the low speed of the sound). In slotted FAMA, nodes stay idle until they have to transmit packets or sense the channel. 


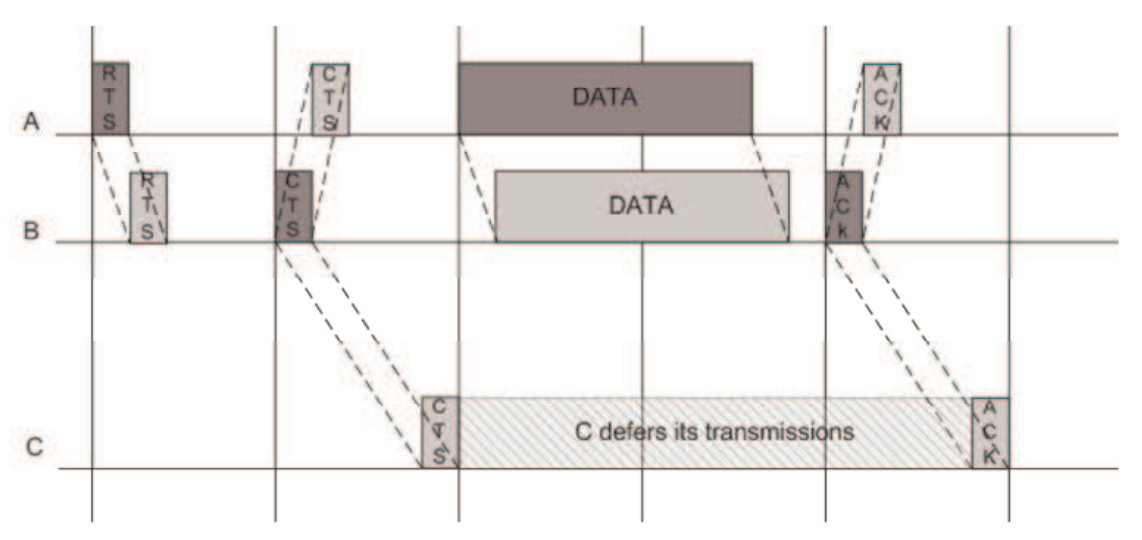

Figure 2.7: Slotted FAMA [21].

Neighbors of either sender or receiver know if they can send information without creating collisions with the sender or the receiver packets. The process of sending packets, either control or data packets, is based on time slots and the data packet transmission is successful when the sender receives an ACK control packet. The communication process starts when a node wants to send data packets to a receiver and it senses the channel and does not detect a carrier. At this point, it has to wait until the next time slot to send an RTS control packet requiring permission from the receiver for data packet transmission. Once the receiver receives the RTS it has to wait until the next slot to send a CTS control packet to the sender informing that it is accepting the data transmission. All of the sender's neighbors also receive the RTS packet. In the same slot that the sender receives the CTS, all of the receiver's neighbors also receive the CTS packet, and in the next slot the sender will start to transmit data packets, as seen in Figure 2.7.

If during the slot time the sender did not receive a CTS, a collision is assumed and the sender will go to a backoff state for a random number of slots. Once this random number of slots is covered, the channel is sensed and an RTS will re-send if no carrier is detected. If a carrier is detected in the channel during a backoff state in the node, the node changes immediately from idle to receiving and executes the operations required in this state. 
Once the operations are ended the node will go back to its backoff state, re-starting its backoff time. After the packet is received, errors are verified using a standard cyclic redundancy check (CRC) algorithm. If everything is correct, an ACK control packet is sent, otherwise a NACK control packet is sent, indicating that the packet needs to be retransmitted (the retransmission is done in the next slot after the sender receives the NACK). A priority scheme must be used to avoid collisions among the packets (CTS and RTS must have the highest priority) and to get a better throughput. The priority in sending packets is also important for getting fairness in underwater communications when remote transmissions are involved.

This protocol presents high complexity and the synchronization process can be costly, especially for dense ad hoc networks. Routing algorithms to update routing tables, and algorithms for network maintenance are also an issue in Slotted FAMA. As a result, scalability has not been well addressed in this protocol.

\subsubsection{UWAN-MAN, an Energy-Efficient MAC Protocol for Underwater Acoustic Wireless Sensor Networks [22]}

One of the objectives for this protocol is to solve the synchronization problem in underwater networks taking into account dense networks with small spacing among its nodes. In addition, in UWAN-MAC the idea of "sleep mode" in every node has been implemented to save energy. The process of getting transmission between nodes in the network with this protocol is divided into an initialization period and a transmission period, and starts with the determination of "listen cycles" to synchronize the schedule for later transmissions in the underwater network. 
In the initialization period every node broadcasts a control packet (SYNC signal that includes its transmission cycle period) at the beginning of its cycle period, goes to its sleep mode turning off its transceiver (saving energy) and it will wait until the end of its second cycle period for the SYNC signal of its neighbors. During the transmission period a node will tell its neighbors that it is going to transmit again after that period of time. The data transmission period starts after the initialization period is complete, which means that every node knows the transmission schedule of its neighbors and when they have to wake up again to receive data from their neighbors. The transmission data packet has a header that is divided into three parts related with the actions that must be taken for the nodes that receive this packet: data, missing and SYNC. The data field represents the destination of the packet. Missing field represents the list of neighbors of the node which have not received its signal.

The SYNC field permits the node to inform its neighbors of changing its cycle period for a new one and as a consequence, its neighbors will change their wake-up times for that node. Once the node ends its data transmission, it goes into an idle listening mode in order to save energy; if it hears something it is going to change its state to a receive mode (this step is for taking into account new nodes that can join the network). When a new node appears in the network, this node is going to send a HELLO message as soon as it receives a packet from some node in the network. This HELLO packet has a time stamp containing information about its transmission schedule. Nodes that receive this packet are going to send back an ACK control packet in their next data transmission time confirming that they have received the HELLO, and update their transmission schedule with the new node.

When a node does not receive data at specific scheduled wake-up time, two things may have happened: either there is a bad channel condition from the sender or the sender had 


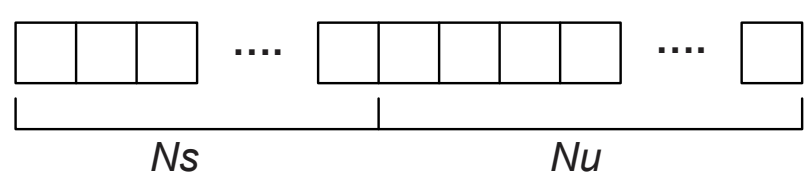

Figure 2.8: Hybrid protocol frame structure [3].

a failure. The receiver node is going to update its missing list and an announcement of this situation will be sent. If the problem was a bad channel condition, the sender is going to receive the missing list, decode it and know that it has to send a HELLO message in its next cycle period, re-scheduling its wake-up time corresponding to the sender. If the problem was that the sender failed, it will not send a HELLO and the receiver will wait for the HELLO for two consecutive cycles of the sender and later it will delete the wakeup time corresponding to the sender (this also saves energy).

The main problem with this protocol is that it uses sleeping modes to save energy, which works perfectly for sensor networks, but not for mobile nodes in which connections among the nodes must stay active all the time in order to avoid some nodes losing communications with the others.

\subsubsection{A Hybrid Medium Access Control Protocol for Underwater Wireless Net- works [3]}

In this protocol it is assumed that all nodes in the network can hear each other and listen to the channel. It works with slotted frames that include scheduled and unscheduled periods combining TDMA with an unscheduled channel access method, to provide low energy consumption by reducing collisions. With scheduled periods collisions are reduced and with unscheduled periods the protocol is adapted to changing traffic conditions. The scheduled period permits nodes to distribute state information. As shown in Figure 2.8, 
the frame is divided into two different structures. The first portion of the frame is divided into $\mathrm{N}$ scheduled slots that use the TDMA protocol, each of those slots will be assigned to one node in the network for transmissions for a long time. The second portion of the frame is divided into $\mathrm{Nu}$ unscheduled slots that will be assigned based on a distributed approach; this assignment will be for a short time and can be used for several nodes in different frames.

For transmissions, the defined time slot covers the maximum length packet plus the longest expected propagation delay, ensuring the nodes will not have problems to completely receive a packet before another node starts to transmit [3]. It is assumed that the nodes use high quality clocks and synchronization protocols and will have slot synchronization with their neighbors. This is important since the medium is acoustic and the channel conditions vary over time [23].

One disadvantage is the assumption of quality clocks and synchronization protocols, which is a problem for underwater networks. Also it is assumed that all nodes will have slot synchronization with their neighbors, and there is no process defined in this protocol to do this.

\subsubsection{T-Lohi, a New Class of MAC Protocols for Underwater Acoustic Sensor Networks [24]}

T-Lohi or Tone Lohi is a reservation-based protocol for underwater acoustic sensor networks. It was designed as an energy-efficient MAC protocol for short range acoustic networks, to exploit low power wake-up hardware whenever energy conservation is needed, and to reduce the propagation delay in underwater transmissions. The energy is conserved 
in two ways: the first by doing data reservations in order to ensure no collisions in data packet transmissions, and the second by using a wake-up tone receiver hardware mechanism to solve reservation contentions by allowing listening with low power for wakeup tones.

The T-Lohi process is done first by executing a tone-based reservation and after that, a data transfer. In the reservation period (contention period), nodes that want to send data packets through the network compete to get and reserve the channel sending a short tone. After that, they listen for a period of time called the contention round (CR) to know whether the reservation was successful or not. Nodes that hear this tone will back off in the data transmission period and the node that obtains the right to transmit will have a data slot reserved. If there was only one node competing for the channel in the CR, the reservation period (RP) ends and the channel is assigned to it. If several nodes were competing in the $\mathrm{CR}$, each of them detect contention and will retransmit again in another CR extending the RP, until one of the nodes gets the channel.

In the data transfer period, a wake-up tone is sent by the sender to wake up the receivers. Once the wake-up tone is received, nodes have to scan the data channel to detect whether there is a preamble or not. If a preamble was not found in the channel the tone is considered as a contention indicator (a low power tone receiver developed by Wills et al. [25] is used by T-Lohi), otherwise nodes will have to decode the packet received and verify if they are the destination. If they are the destination, they will be switched to a receive mode, otherwise they will go back to sleep. There are different versions of T-Lohi depending on the reservation mechanism implemented: synchronized T-Lohi (ST-Lohi), conservative unsynchronized T-Lohi (cUT-Lohi), and the aggressive UT-Lohi (aUT-Lohi). This protocol is more adequate for sensor networks, since nodes must go to a sleeping state for saving energy consumption. In some cases when the traffic in the network in- 
creases, due to the absence of CTS control packets at the receiver, the hidden terminal problem is present causing an increase of collisions and as a consequence the throughput of the network decreases.

\subsubsection{A MAC Protocol for Underwater Sensor Networks [26]}

This protocol works with a single transceiver and it is distributed. The authors improve the network efficiency by proposing a multi-channel MAC protocol based on the MACA protocol in [27]. It utilizes one channel for control packets (common code) and several channels for data packet transmissions where different spreading codes are applied. By using several CDMA schemes, nodes can simultaneously transmit by overlapping in time and space domains. All nodes in the network are assigned to the same common channel (common code) and the common code is monitored for any packet arrival. Once the source sends the RTS and receives the CTS (RTS-CTS handshaking), the optimal spreading code in which the data packet is going to be transmitted is chosen for both nodes (sender and receiver). After receiving the data packet, the destination despreads the received signal and retrieves the data.

At the end, the destination node will send an ACK packet to the transmitter. An example of this process is shown in Figure 2.9 in which the common code is $c$, and the code selected for data transmission is $C t l$. After node 2 sends the CTS to node 1 , the $C t l$ code is chosen by the transmitter for data transmission.

As in other underwater MAC protocols, one of the disadvantages is the capture problem. In addition, scalability problems when a node decides to join or leave from an underwater network are present. 




Figure 2.9: A MAC protocol using RTS/CTS handshaking [26].

Other MAC protocols designed for underwater networks are multi-cluster protocol for ad hoc mobile underwater acoustic networks [28], a distributed CDMA medium access control for underwater acoustic sensor networks [29], distributed MAC protocols for underwater acoustic data networks [30], R-MAC [31], and an energy-efficient MAC protocol for underwater wireless acoustic networks [32].

\subsection{Logical Link Control (LLC) Layer}

Protocols in this layer have been created to improve the throughput in the network, and to reduce the amount of packets in error. Due to problems like the long propagation delay present, bad quality channel (several packets in error) and very low throughput efficiency in underwater communications, traditional LLC protocols need to be modified in order to be more adequate for underwater communications. There are some protocols that have been implemented to improve the problems just mentioned. The classification of the logical link protocols and some examples of them shown in Figure 2.10 will be explained in the following sections. 


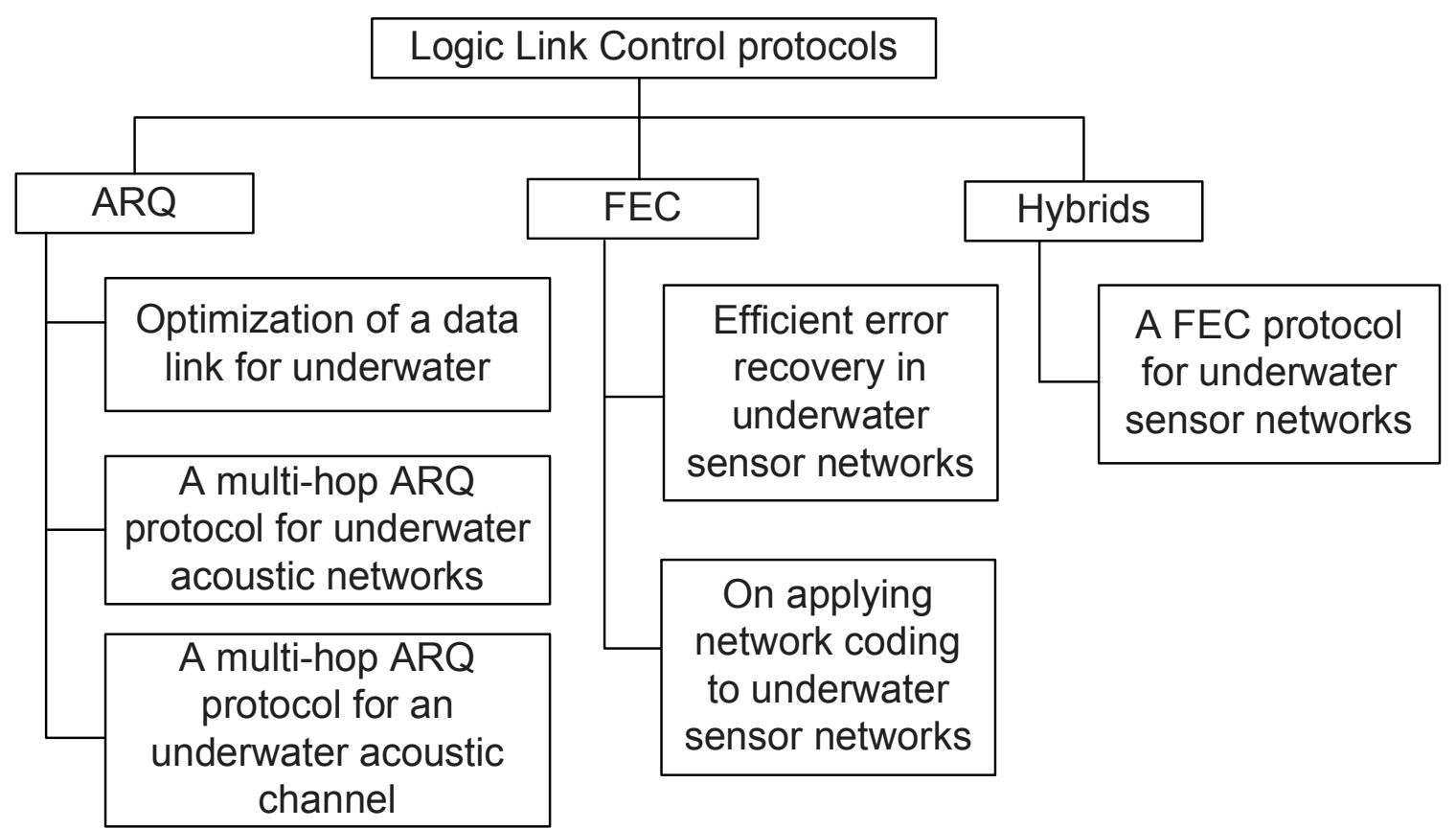

Figure 2.10: Classification of logic control protocols.

\subsubsection{Logical Link Control Classification}

Logical link control protocols are classified depending on the actions required for the packets arrived to the receiver with errors, either only detection or detection and correction. These protocols are classified in Automatic Repeat Request (ARQ), Forward Error Correction (FEC) and hybrid protocols, as shown in Figure 2.10.

\subsubsection{ARQ Protocols}

ARQ protocols have been designed for data error detections of packets that arrive to the receiver [33]. Once errors are detected, packets are retransmitted until they arrive without errors or the maximum number of retransmissions is achieved. 
The problem with these protocols is the amount of packet retransmissions that can be produced when the channel error rate increases. The increase of packet retransmissions will cause the throughput in the network to decrease trying to maintain high reliability in the network.

In [33] it is explained which is the optimal blocklength for packets in order to reduce the data retransmissions. It is better to have the largest blocklength to minimize the time wasted sending acknowledgments and the associated delays, but also it is better to have the smallest blocklength to minimize the error probability of the packet sent and to minimize the time wasted in retransmissions.

As a consequence of obtaining the optimal blocklength, the throughput efficiency is maximized; in other words, the wasted time is minimized. The optimization of the throughput efficiency varies depending on the type of ARQ protocol and it will be explained in the following section where some examples of underwater logical link protocols are shown. ARQ protocols are divided into stop and wait, go back N, and select and repeat protocols.

- Stop and Wait Protocols. In stop and wait protocols, the transmitter sends one packet and it will only transmit the next one after receiving a positive acknowledgment from the receiver, telling it that the packet received was correct. If the transmitter either does not receive the acknowledgment (timeout) or receives a negative one, it will retransmit the packet to the receiver. This process will be repeated until a positive acknowledgment is received. This protocol is easy to implement, however one problem is the total time spent when a message to send is split into small packets (only one packet can be sent at a time).

Another problem is the time lost between sending a packet from the transmitter and receiving the positive acknowledgment, because in that time the transmitter cannot 
send another packet. Furthermore, a deadlock situation will occur if the transmitter does not receive the positive acknowledgment, or if the receiver does not receive the packet or the packet comes with errors all the time. If any of these situations occur, the packet will have to be retransmitted, creating an infinite loop, unless a maximum number of retransmissions is defined.

- Go Back N Protocols. Go Back N is a sliding window protocol. This protocol works using a window of size $\mathrm{N}$ to specify the maximum number of packets being sent (when available) without waiting for an acknowledgment. The protocol easily handles bad frames and lost frames by maintaining a list of the frames transmitted that remains unacknowledged and most recently transmitted frames [34]. In order to have the list, a buffer is required at the receiver. If error occurs, sender transmits all frames from frame in error and receiver discards all others. The implementation of this protocol can be difficult, but the most challenging part is to find the optimum timeout value that provides efficient transmission for all error rates.

Smaller timeout value seems to be an excellent decision on higher error rates but it is better to use a marginally higher value to optimize them. Another problem in this protocol is the high number of frames retransmitted, which generates bandwidth waste and a longer time to complete transmissions. The cause of this is that for every lost frame, all the frames in the window transmitted previously must be retransmitted.

- Selective Repeat Protocols. As in the Go Back N, a window size is used in Selective Repeat protocols. Windows are used not only in the sender but also in the receiver and those window sizes must be equal. The difference with Go Back $\mathrm{N}$ are that the receiver accepts buffer out-of-order frames arriving without errors, and a 
retransmission mechanism is used to ask for the retransmission of specific packets. Every frame sent by the transmitter has the data information and an additional sequence number. By the inclusion of he sequence number, the receiver knows the earliest frame not received, and it will send back an ACK with the corresponding number.

The sender transmits frames until its window is empty, moving its window for every packet acknowledged in the receiver. Later, it will re-send frames which did not arrive and then will continue where it had left off. By the time the sender is sending frames, the receiver is also filling its receiving window with those frames, frames that have been verified.

Once the receiving window is full, all frames will be sent to the upper layer [34]. For wired networks, all of them can be applied, but selective repeat protocols are the most efficient (wired networks are full duplex). In underwater networks, because of the way that packets can be transmitted via transducers, only half duplex communication can be done, meaning that stop and wait protocols only are used.

\subsubsection{Forward Error Correction Protocols}

These protocols are applied to both detect and correct errors. They add redundant information to the original frames at the sender. This redundant information is used to reconstruct approximations or exact versions of packets with errors. Once the packet is received by the receiver, a FEC technique is applied to both detect and correct errors. This process helps to decrease the number of retransmissions that could be done by the sender due to immediate corrections of errors executed by the receiver. 
These techniques also reduce the time for transmissions between sender and receiver because the sender avoids waiting for the round-trip time propagation delay needed to receive the non acknowledgment (NAK) control packet, and as explained before, frames are fixed at the receiver [34].

\subsubsection{Hybrid Protocols}

Hybrid protocols combine ARQ and FEC techniques. They were created to obtain a more efficient process to decrease the number of retransmissions that can be done by the sender, compared with ARQ and FEC protocols. Different strategies are applied during the packet transmission, to identify when detection schemes are used instead of correction schemes.

\subsubsection{Underwater Logical Link Control Protocols}

A description of some LLC protocols and mechanisms for underwater communications are shown in Figure 2.10, and are explained next.

\subsubsection{Optimization of a Data Link Protocol [35]}

A comparison among some ARQ protocols are done in this paper. In underwater acoustic channels, the efficiency of the ARQ schemes is limited by both the poor bit error rate (BER) performance and the long delay generated by the low speed of sound propagation, which is $1500 \mathrm{~m} / \mathrm{s}$. In [35] statistical analysis is made to maximize efficiency of ARQ schemes based on finding the optimal packet size to be sent as a function of bit error 
probability. The statistical analysis is applied to three different versions of the stop and wait protocol which are the well known stop and wait, and versions in [33] and [36]. It is assumed that each packet has $N=N_{d}+N_{o h}$ bits, where $N_{d}$ is the number of data bits, and $N_{o h}$ is the packet overhead whose number of bits is at least the number of bits used for the Cyclic Redundancy Check (CRC) function. $T_{p}=N T$ is the packet duration where $T=1 / R$ is the bit (symbol) duration, and $R$ represents the bit (symbol) rate. Moreover, $T_{\text {sync }}$ is the synchronization preamble which precedes the group of packets transmitted. $T_{d}=l / c$ is the propagation delay, $l$ is the distance transmitter-receiver, and $c=1500 \mathrm{~m} / \mathrm{s}$ is the speed of sound underwater. If $m$ represents the number of packets to be transmitted, then the time needed to transmit them and the reception of the corresponding group of acknowledgments is

$$
T(m)=m\left(T_{p}+T_{a c k}\right)+T_{w}
$$

where the total waiting time is

$$
T_{w}=2\left(T_{s y n c}+T_{d}\right)
$$

and the duration of an acknowledgment is negligible with respect to the packet duration, in other words $T_{a c k}<<T_{p}$. The timeout of an stop and wait protocol that transmits $m$ packets has to be the same as the round-trip time $T(m)$ in order to obtain the best efficiency. Other concepts applied are the throughput efficiency of ARQ protocols that is the ratio of useful packet time and the total time spent on the average for a successful packet transmission in which the average is over the number of retransmissions, and $p$ that is the packet error probability. Instead of transmitting one packet every time like in the traditional stop and wait protocol, in protocols in [33] and [36] a window of $m$ packets are sent in each transmission. It is shown that the Morris version [33] presents the best throughput efficiency due to the amount of new packets transmitted in every window. 




Figure 2.11: One-dimensional n-hop acoustic channel [37].

\subsubsection{A Multi-Hop ARQ Protocol [37]}

This protocol is an opportunistic acknowledgment scheme created for stop and wait ARQ protocols and works over two different types of underwater networks: one-dimensional n-hop acoustic channel and two-dimensional multi-hop acoustic sensor networks, as seen in Figure 2.11 and Figure 2.12 .

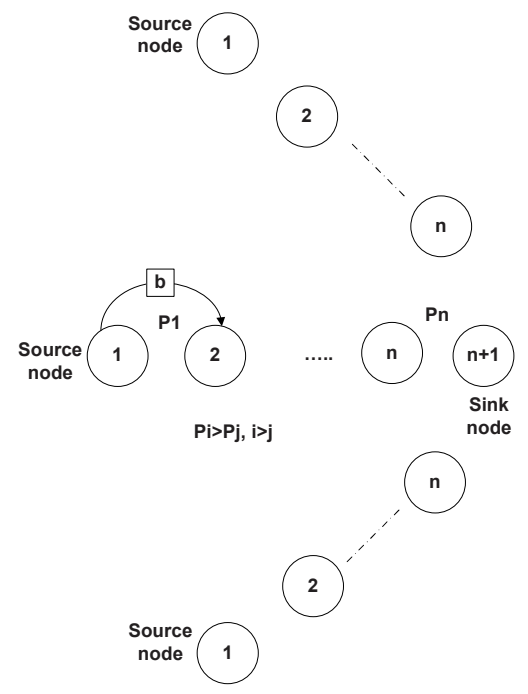

Figure 2.12: Two-dimensional multi-hop acoustic sensor network [37].

The design proposed is a per-hop hybrid implicit/explicit acknowledgment scheme over a multi-hop channel taking into account that acknowledgments in general are sent from the receiver to the transmitter via two different procedures, Implicit Acknowledgment 
(IMP) or Explicit Acknowledgment (EXP). To understand how IMP works, consider a transmission from node $j$ to node $j+1$. If node $j$ hears that the node $j+1$ is forwarding its packet, there will be an implicit acknowledgment from $j+1$ to $j$. If node $j+1$ does not forward the packet received from $j$ after a timeout, meaning that the node $j$ is not acknowledged, then node $j$ will retransmit the packet. EXP happens when node $j$ sends a packet to node $j+1$, and waits for the explicit acknowledgment that $j+1$ has to send it. Node $j+1$ transmits the acknowledgment if its previous packet transmission was acknowledged, except the node $n+1$, which is the last node and only has to send (ACK) packets. The purpose of using either IMP or EXP in the hybrid design is to maximize the latency efficiency, and/or the energy efficiency for data delivery in multi-hop acoustic network systems. Another purpose is to reduce unnecessary transmissions.

Some of the disadvantages in this protocol are the hidden, deafness and capture problems still present. The poor packet delivery ratio obtained using the traditional stop and wait protocol and taking into account the bad quality channel in underwater networks is another problem. In addition, the long propagation delay present in underwater communications negatively affects the throughput efficiency, especially when stop and wait protocols are applied.

\subsubsection{FEC-Based Reliable Data Transport Protocol for Underwater Sensor Net- works [38]}

The protocol that is called Segmented Data Reliable Transport (SDRT) is a hybrid approach of ARQ and FEC. SDRT is in charge of reconstructing lost packets instead of error-correction in packets. The process of sending packets starts with grouping the data packets to send in blocks, in which a window of $m$ blocks is sent by the transmitter. This 
number guarantees that the reconstruction process can be done in the receiver, and it is obtained by estimations taking into account the speed of the mobile nodes, sound propagation speed, available bandwidth and distance between sender and receiver.

Once the blocks are generated, they are encoded using the FEC protocol called Tornado [39] and transferred into the network. Data packets are forwarded block by block and hop by hop by intermediate nodes. After a node starts to receive blocks from the sender, it waits for the window of encoded packets needed that guarantees the reconstruction of the data packets. When the window of $m$ encoded packets is sent, the sender does not stop transmitting subsequent packets; it continues sending them but at a slow data rate, waiting for the acknowledgment from the receiver related to the window sent. At the receiver side, once the window is received, it tries to reconstruct the encoded data packets. If this is possible, it will send back an acknowledgment, and these packets will be encoded and forwarded to the next hop. After receiving the acknowledgment, the sender will stop the process of sending subsequent packets.

One of the problems in this protocol is that the estimation of the number of blocks depends on the available bandwidth. With a poor bandwidth, which is common in underwater networks, the number could be one, executing the traditional stop and wait process in which a node that sends a packet, cannot do anything else until it receives an acknowledgment. That means time will be wasted for nodes when they are waiting for acknowledgments, especially in underwater acoustic networks where long propagation delays are present. Other problems are not only the throughput of the network but also the packet delivery ratio, which decrease if the number of packets also decrease, and the scalability in the network. 
Other LLC protocols developed for underwater networks are: a multi-hop ARQ protocol for an underwater acoustic channel [40], efficient error recovery using network coding in underwater sensor networks [41] and on applying network coding to underwater sensor networks [42]. 


\section{Chapter 3: 2MAC: A Multichannel MAC Protocol}

The proposed MAC protocol $2 \mathrm{MAC}$ has been created in order to reduce typical problems present in wireless networks like collisions, and hidden and exposed terminal problems, and to improve the throughput performance in the underwater channel.

This chapter describes $2 \mathrm{MAC}$ in detail, the most adequate network topology needed for this protocol, how the channel assignment works having OFDMA at physical layer, possible scenarios where 2MAC works, and comparisons with other traditional MAC protocols using different backoff approaches.

\subsection{MAC Description}

2MAC is a contention-based MAC protocol based on the MACAW protocol [43]. It has been designed to improve the performance efficiency in underwater ad hoc acoustic networks having neighbors located in different sub-channels.

2MAC uses a four-way handshaking access method (RTS/CTS/DATA/ACK), a new control packet called BTS (Blocked To Send), an Adjusted Response time (ARS) to wait for signals from both neighbors, and a listen/contention time to exchange data, as shown in Figure 3.1. In every transmission process, $m$ data packets are transmitted through each 


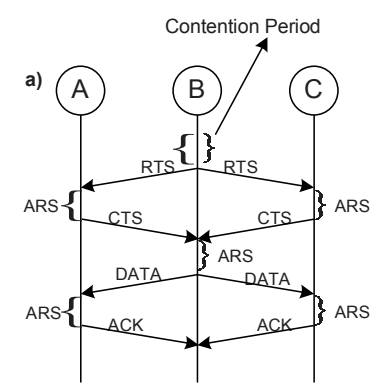

(a)

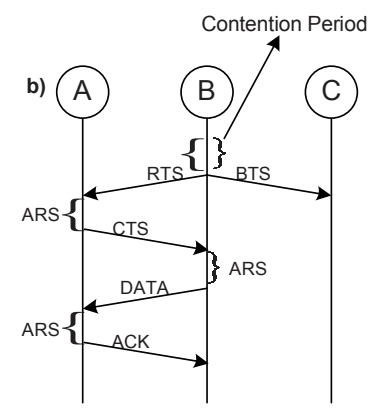

(b)

Figure 3.1: 2MAC transmission process.

channel. Short Inter-Frame Spacing (SIFS) and Distributed Inter-Frame Spacing (DIFS) are also used in the packet transmission process as in IEEE 802.11.

BTS is a control packet to inform that a channel will not be available for communications with the corresponding neighbor. Once a sender decides to start a transmission and just one channel is going to be used, the sender starts a handshaking process through this channel but at the same time transmits a BTS in the other channel to tell its other neighbor for how long the channel is going to be unavailable. This is shown in Figure 3.1(b) in which Node A has packets for A but not for C.

The proposed protocol works with the assumption of existing OFDMA in the physical layer that creates multiple communication channels that can be used simultaneously by the MAC layer (there is just one transceiver in each node). 2MAC relies in OFDMA at the lower layer and defines two subchannels per node for simultaneous communications with its neighbors, and three channels at the same time for the entire network. The assumptions for 2MAC are outlined below:

- A single half-duplex transceiver

- OFDMA in the physical layer 
- A network with a linear or polygon topologies, for swarming purposes

- Distance between nodes must be known and stay without variations by having a control system in each AUV

- Three channels used simultaneously in the network

- Every node has two neighbors and each of them works in a different channel

\subsection{Network Topology and Channel Assignment}

By using several subchannels per node simultaneously, it is possible to have different topologies, especially linear or polygon topologies, and to transmit information to each of the neighbors without creating collisions. Figures 3.2 and 3.3 show linear and hexagon topologies with the assignment of three sub-channels that allow simultaneous transmission without interference. For example, in the linear topology, node A has B and C as its neighbors, it communicates with B through channel 2 and with $\mathrm{C}$ through channel 1. C and B can communicate with A and D respectively at the same time, without experiencing the exposed terminal problem since A does not share the same channel with $\mathrm{C}$ and $\mathrm{B}$; similarly, A and E can communicate with C simultaneously without colliding. A similar situation will happen having either hexagon or nonagon topologies, as seen in Figures 3.3 and 3.4.

There is a relation between the number of channels in the network, two as a minimum, and the number of sides that a polygon topology can have. Using three channels in the network but two different per node, it is possible to have polygon topologies in which their number of sides is a multiple of 3. 


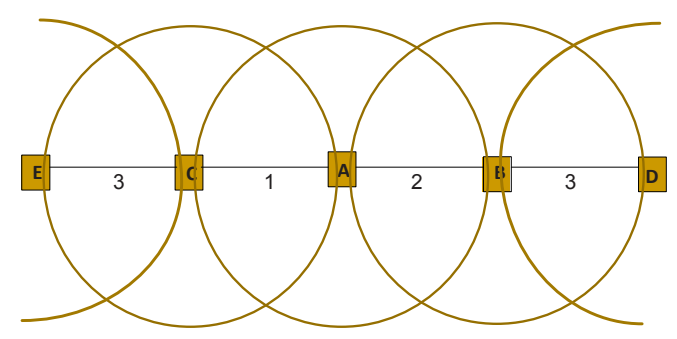

Figure 3.2: Linear topology.

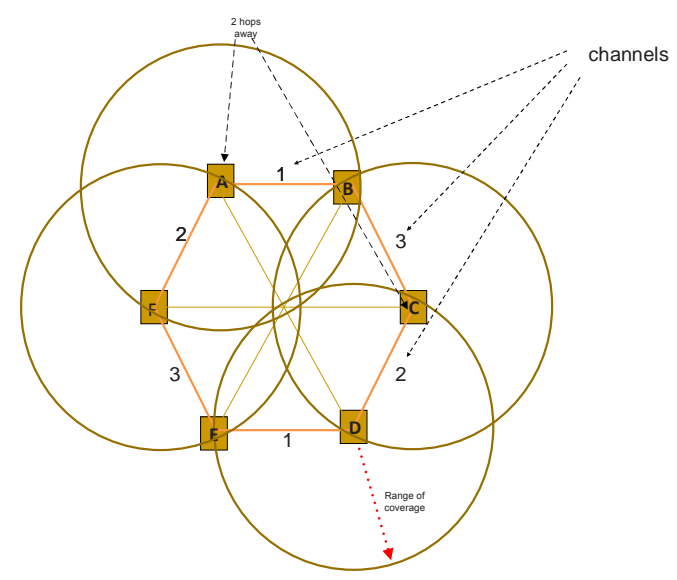

Figure 3.3: Hexagon topology.

The optimal number of channels per network required to set in a polygon topology can be obtained using Equation 3.1, in which $S$ represents the number of sides that the polygon topology is desired to have and $\mathrm{CH}$ is the optimal number of channels needed. The value of $\mathrm{CH}$ is such that $n$ is the maximum quotient value. As an example for the hexagon topology shown in Figure 3.3, 3 or 6 channels can be defined, but using only 3, a maximum value $n$ is obtained when $S$ is divided by $C H=3$, where nodes using the same channel in a topology with $\mathrm{CH}$ channels will be $\mathrm{CH}-1$ hops away from each other, avoiding collisions. With 3 OFDMA sub-channels the maximum value is obtained for $n$, and the communication among the nodes can be effectively done.

Another example is having a dodecagon topology in which a 3-channel, 4-channel or 12channel network can be set up. With 3 channels defined by OFDMA the maximum value 




Figure 3.4: Nonagon topology.

is obtained for $n$, and the communication among the nodes can be effectively done. Linear topologies are also possible and they need only a 3-channel network; it does not matter how many nodes will be in the linear network.

$$
n=\max \left\{\frac{S}{C H}\right\}
$$

\subsection{Scenario}

With OFDMA working at the physical layer, every neighbor will use a different channel, two channels per node, and the total amount of channels used simultaneously for all nodes will be three (for explanation purposes the network will be a polygon topology), as seen in Figure 3.3. It is important to highlight that by using $2 \mathrm{MAC}$ over linear or polygon topologies, hidden and exposed terminal problems, capture and deafness problems are eliminated. These problems will happen only when two adjacent nodes try to communicate with each other at the same time by sending an RTS packet. This situation will be explained later. 
In this type of design due to the range of coverage in every node (even though there is just one transceiver per node), A and C can start a conversation simultaneously with B without collisions at the receiver (no hidden terminal problem); this is because the communication is through different channels. Also, $\mathrm{C}$ can start transmitting to D without taking into account what is happening between $\mathrm{A}$ and $\mathrm{B}$ for the same reason (the exposed terminal and capture problems are avoided). The most important restriction is that nodes using the same channel must be 2 hops away from each other. In the next section it will be shown how 2MAC works, taking advantage of OFDMA over these topologies.

\subsection{MAC State Transitions}

2MAC has the following ten states: Idle, Channel Assignment, Contention (Listen), Waiting for CTS, Receiving RTS, Waiting for ACK, Waiting for Data, Backoff, Adjusted Response, and Blocked to Send. Figures 3.5 and 3.6 show the 2MAC sender and receiver state machines, respectively. The states displayed in the figures that represent the sender and receiver process are explained next.

\subsubsection{Idle State}

When a node receives an RTS through one of its channels in this state, it goes to the Receiving RTS state to check if an RTS packet is coming from the other channel. It stays in the Idle state until it has packets to send, when it goes to the Channel Assignment state. In the Idle state, a node can receive not only RTS but also BTS packets. If the node receives a BTS packet, it blocks the corresponding channel for the time defined in the BTS, 


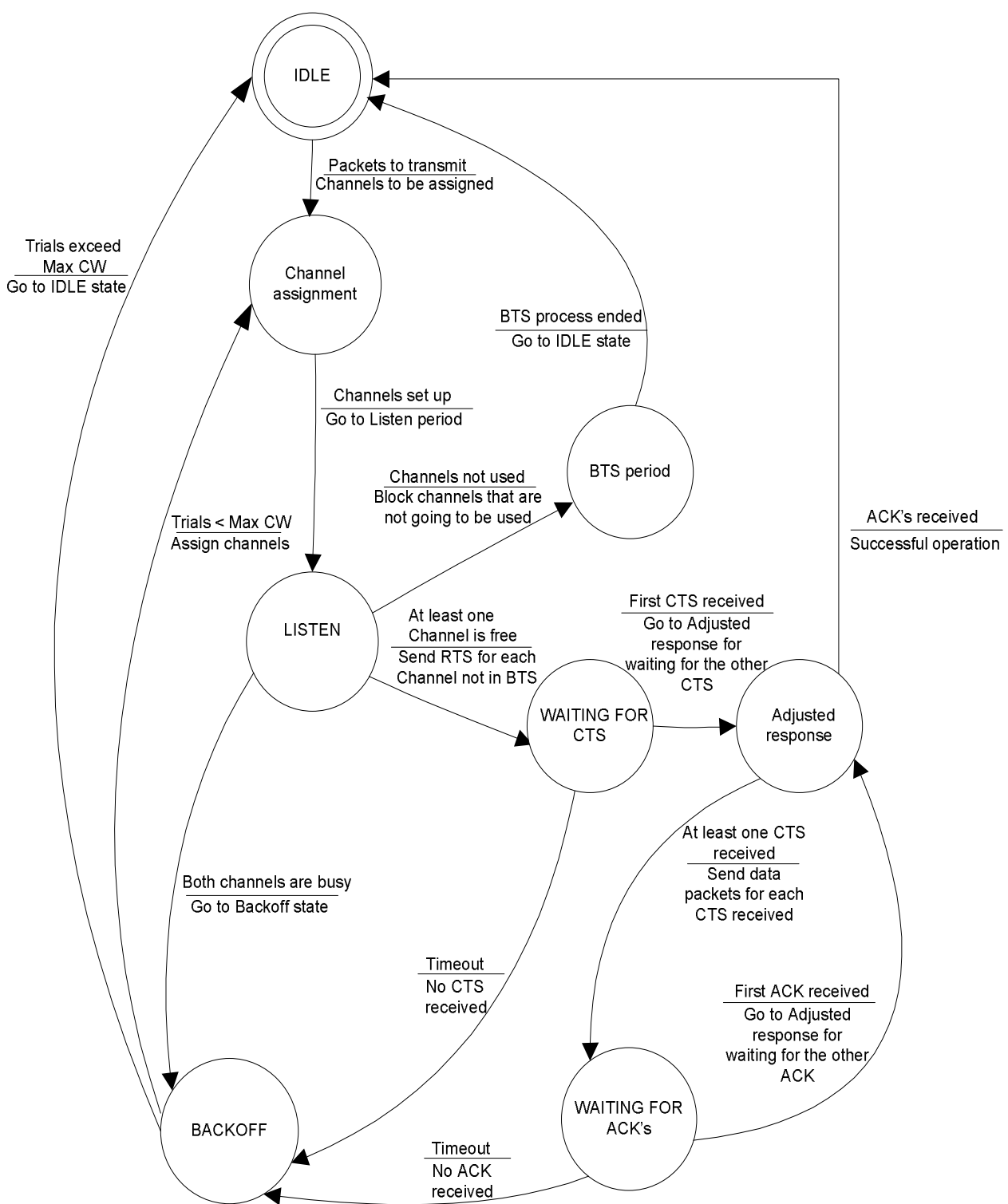

Figure 3.5: Finite state machine of 2MAC process at the sender. 


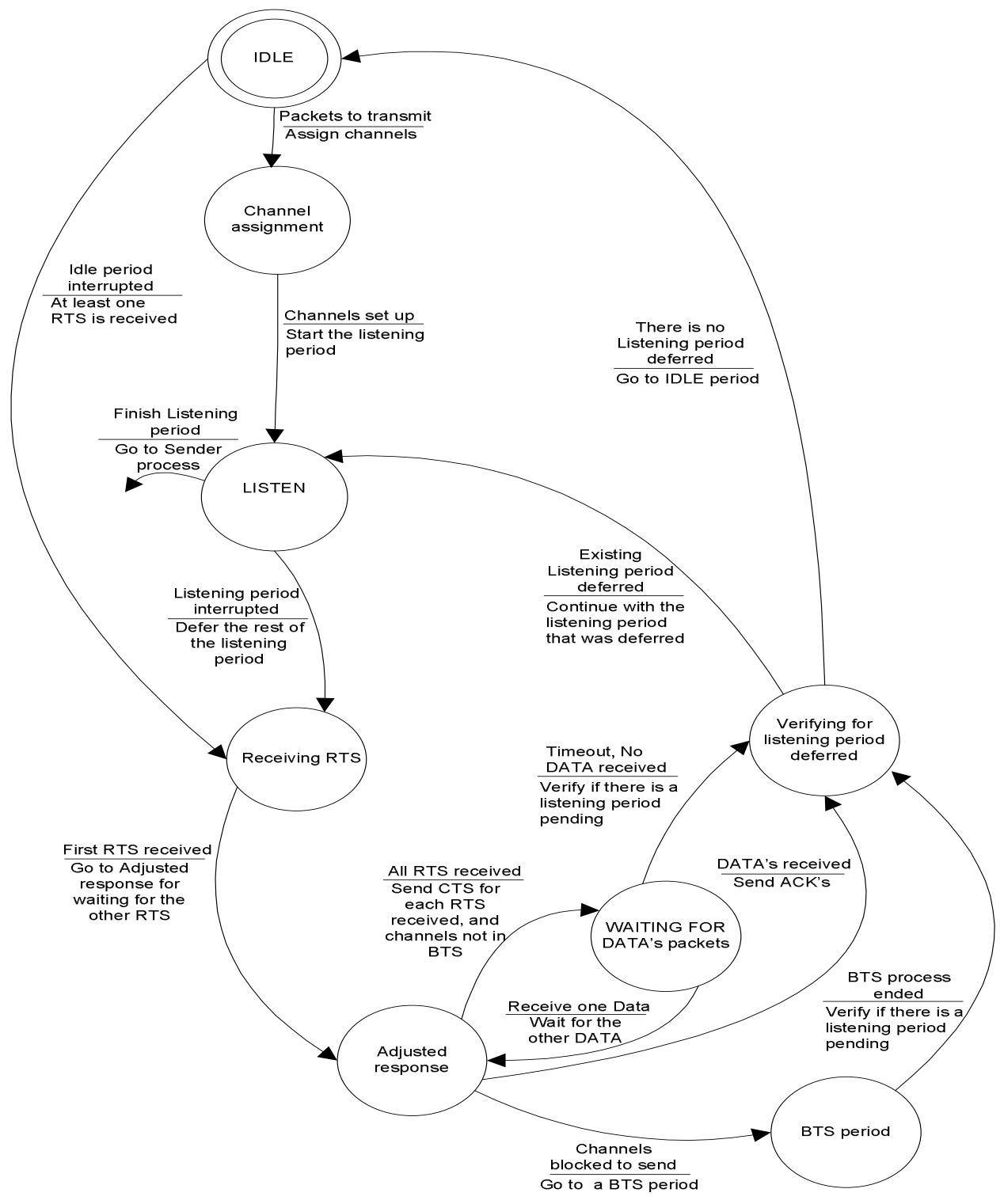

Figure 3.6: Finite state machine of $2 \mathrm{MAC}$ process at the receiver. 
and only the other channel is available for transmissions during that time. When a node receives a BTS packet from both channels, it goes to the Blocked to Send state.

\subsubsection{Channel Assignment State}



Figure 3.7: 2MAC, transmission diagram.

Since every node has two neighbors and each of them has a different channel assigned, two BTS fields are used to avoid collisions, one for every channel (similar usage like NAV in IEEE 802.11), as seen in Figure 3.7. Once packets arrive from the upper layer, their destinations are verified and the corresponding channels are selected and activated depending on their channel BTS values. If two data packets must be transmitted at the same time (one for every neighbor), both BTS are verified, otherwise only one data packet is transmitted and the corresponding channel BTS value is analyzed. A nonzero BTS value means that the channel is unavailable to send packets because that neighbor is already using it. 
Three possibilities can happen. The first one is when two $M$ data packets must be transmitted simultaneously in different channels and at least one of the corresponding BTS values is zero. If both BTS are zero, all channels are activated and the transmitter will go to the Contention state in both channels, to see whether neighbors are transmitting or not and to start a transmission process later. Otherwise, the data packets that correspond to the blocked channel, the channel with the nonzero BTS value, will go back to the queue for another transmission process, and the node will go to the Contention state only for the channel with the zero BTS value; this channel is activated. In the case that both BTS have nonzero values, the node will go to the Blocked to Send state.

The second possibility occurs when two $M$ data packets must be transmitted but both BTS values are nonzero, in this case the node will go to a Blocked to Send state. Once its Blocked to Send state finishes, the node will go back again to the Channel Assignment state.

The last possibility is when just one $M$ data packet has to be transmitted and its corresponding BTS is either a zero or nonzero value. If BTS is zero, the corresponding channel is activated, the other one is put on hold (it will used to send BTS packets), and the node will go to the Contention state, otherwise it will go to a Blocked to Send state.

\subsubsection{Contention (Listen) State}

In this state the transmitter will be listening to the activated channels (either both or just one) for a time equal to a round trip time of a control packet (CTS or RTS), depending on what happened in the Channel Assignment state. If the channel (or channels) are free (no communication occurs during the Contention state), the node sends RTS simultaneously 
to all the activated channels to start a communication process (a BTS will be sent through the activated channel that is not going to do a data transmission), and goes to the Waiting for CTS state. If the channel is busy, the node defers its remaining contention time, and receives the neighbor transmission(s). Once transmission finishes, it goes back to this state to finish the listening period.

The example in Figure 3.8(d) shows a node B finishing its contention period without receiving any packet from its neighbors. Then, a communication process starts with the nodes it needs to transmit data packets by sending RTS at the same time to A and B (due to the presence of OFDMA). When one of the channels is put on hold, meaning there are packets to send to only one neighbor (the other channel was activated), the transmitter will send two different type of packets, the RTS for the neighbor it wants to start a data transmission with, and a BTS packet to the other neighbor, relaying the time that it is going to be busy, as seen in Figure 3.8(a). In Figure 3.8(b), Node B starts a handshaking with A but a BTS was not sent to C because previously, the channel was blocked.

\subsubsection{Waiting for CTS State}

In this state a node waits until either it receives both CTS or a timeout occurs. After receiving the first CTS packet, the transmitter goes to the Adjusted Response state to wait for the second CTS arrival. If the sender does not receive it, it assumes that a collision occurred in that neighbor and the corresponding channel is disabled for data transmissions. When a timeout occurs, the sender enters the Backoff state for a certain amount of time. Once the Adjusted Response state finishes, the transmitter sends the data packets through the corresponding activated channels and goes to the Waiting for ACK state. 


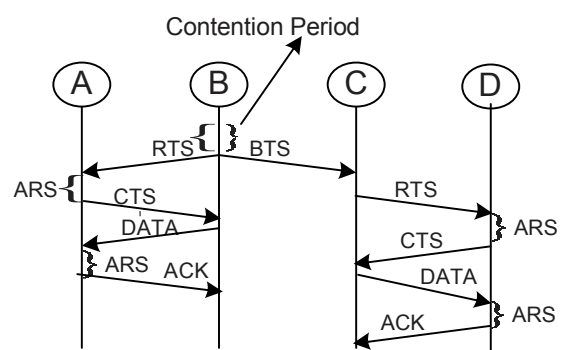

(a)

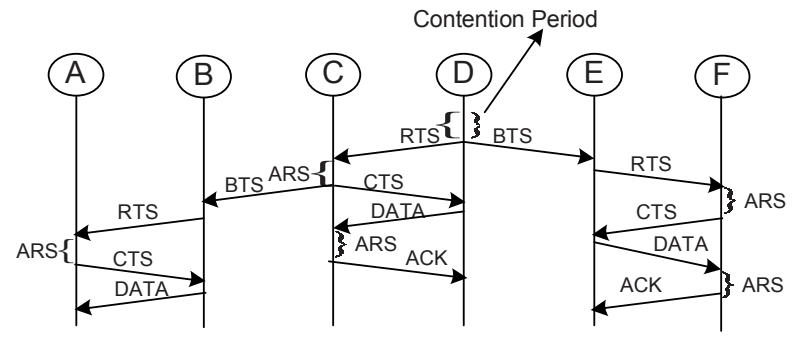

(c)

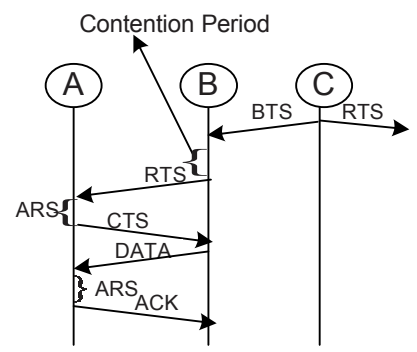

(b)

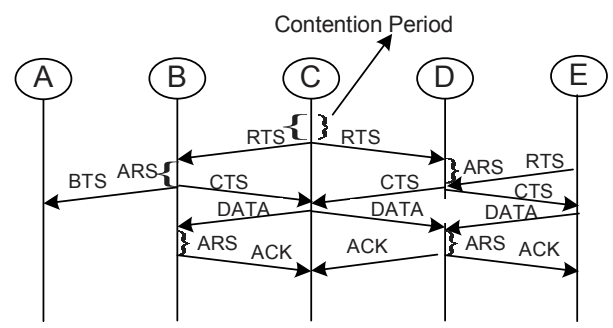

(d)

Figure 3.8: Contention process.

In the example in Figure 3.9(a), both CTS are received simultaneously, then B does not spend time in the Adjusted Response state. However, in Figure 3.9(c) once B receives the CTS from node $\mathrm{C}$ it will be in the Adjusted Response state to see whether or not a CTS packet will come from A. The Figure 3.9(d) shows how a timeout can occur; in this case B will go to a Backoff state.

\subsubsection{Receiving RTS State}

If a node receives an RTS while being in the Contention state, it defers its remaining time until the communication process finishes. In the Receiving RTS state, when the first RTS is received the node goes to the Adjusted Response state to verify that another RTS is coming from the other neighbor. If two RTS are received, a CTS is transmitted through its activated channels simultaneously, otherwise just one CTS is sent to answer the RTS, 




(a)

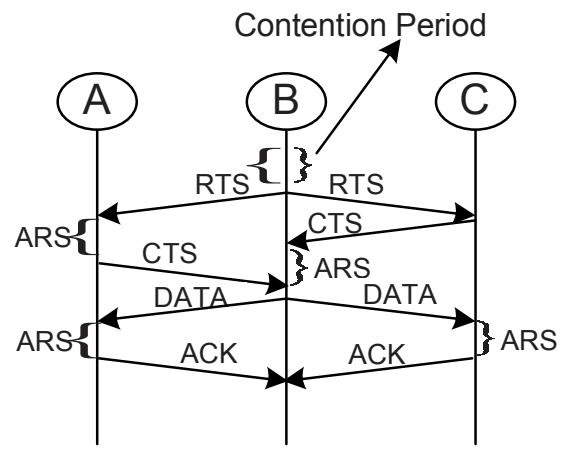

(c)

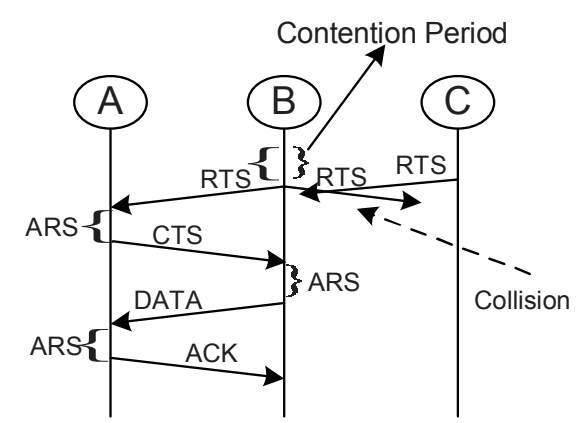

(b)

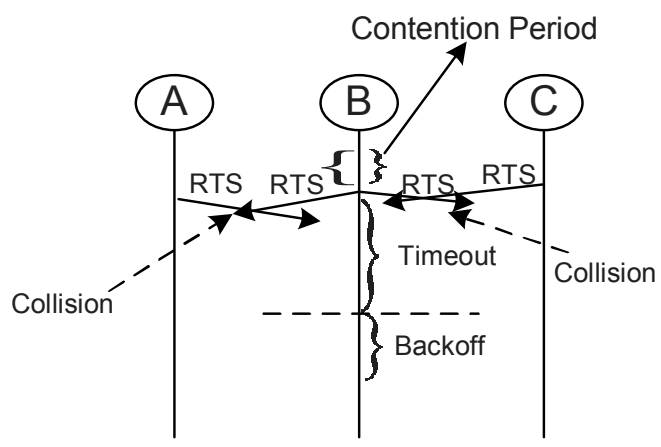

(d)

Figure 3.9: RTS/CTS communication process.

and a BTS is transmitted to its other neighbor carrying the time it will be busy. Once the CTS are transmitted, the node goes to the Waiting for Data state.

As shown in Figure 3.8(c) for example, C receives an RTS from D. Then, after the Adjusted Response period finishes, it sends two packets, a CTS to D and a BTS to A (to indicate $\mathrm{C}$ is going to be busy and the channel with $\mathrm{A}$ will be blocked). In this way, $\mathrm{A}$ and $\mathrm{B}$ and $\mathrm{E}$ and $\mathrm{F}$ can start a transmission without generating any problems with the transmission between $\mathrm{C}$ and D. In the example in Figure 3.9(c), B receives CTS from A and C, and it starts the data packet transmission simultaneously to both nodes. This is possible because OFDMA resides in the lower layer. 


\subsubsection{Waiting for ACK State}

In this state the node waits until either it receives both ACK, a timeout occurs, or just one ACK arrived. After receiving the first ACK packet, if only one channel is activated, the transmitter finishes its transmission process, channels are set up to the available mode, and the node goes to the Idle state. If after receiving the first ACK packet both channels are activated, the transmitter goes to the Adjusted Response state to wait for the second ACK. If the sender does not receive the second ACK, it assumes that a collision occurred in that neighbor, its corresponding data packet will be retransmitted in the next transmission process, the channels are set as available, and the node goes to the Idle state. When a timeout occurs, the sender goes to a Backoff state for a certain amount of time.

\subsubsection{Waiting for Data State}

Once the first data packet is received, the receiver goes to the Adjusted Response state to verify that another data packet is coming from its other neighbor. Otherwise, a timeout occurs and the node goes either to its deferred contention period or to an Idle state. Once the Adjusted Response state finishes, ACK for the corresponding data packets are sent through each activated channel, and the receiver waits until the time defined in its BTS finishes to set up its channels as available and start BTS in zero.

As shown in Figure 3.8(d), node D does not have to go to the Adjusted Response state because it receives data packets from $\mathrm{E}$ and $\mathrm{C}$ simultaneously. Then, it sends both ACK to $\mathrm{C}$ and $\mathrm{E}$ at the same time. 


\subsubsection{Backoff State}

In the case of collisions, nodes execute a Binary Exponential Backoff (BEB) retransmission algorithm for collision recovery that is a modification to the one used in [44]. The sender goes to the Backoff state for a certain amount of time, and the transmitter starts again a Channel Assignment period for a new contention time. Once the Backoff finishes, if the number of retransmissions have reached the maximum allowed number the packet is dropped and the node goes to the Idle state. A node receiving an RTS while in the Backoff period defers its remaining time until the communication process finishes.

\subsubsection{Adjusted Response State}

Nodes in this state wait for a certain period of time called Adjusted Response State (ARS) to see if another packet is coming or not. Since a node can receive packets from its neighbors through different channels, these packets do not necessarily arrive at the same time and a gap is produced between both arrivals. To control this gap, an ARS time is included.

The goal of this state is to get both channels of a node to stay either in the receiving or the sending mode at the same time by using an Adjusted Response period. With this state, a node knows if there was a request or not from both channels and later responds simultaneously through both channels. For example, as in Figure 3.8(d), node D is receiving RTS from its neighbors in different moments. With the Adjusted Response period, D will respond with CTS at the same time to $\mathrm{C}$ and $\mathrm{E}$. This ARS time is defined as a constant value in the $2 \mathrm{MAC}$ protocol, and included as a portion of the timeout (the timeout will be the ARS plus the cost of a control packet round trip time). 


\subsubsection{Blocked to Send State}

A node is in this state when BTS are received from both its channels or just from the activated channel. It uses the largest of the BTS times to block the channel for that period of time. Once the time finishes, the BTS values are reset and the node goes to a Channel Assignment state if there are packets to transmit; otherwise it goes to the Idle state.

\subsection{MAC's Backoff Algorithm}

The well-known Binary Exponential Backoff (BEB) algorithm was designed initially to deal with congestion in 802.3 networks, and now they are also used with 802.11 networks. During congestion epochs, nodes are forced to wait longer and longer after successive collisions by doubling the size of the contention window each time. Although this has been shown to be a good mechanism in wireless local area networks, its direct application in long propagation delay underwater channels might not be a good idea. The long propagation delays in underwater communications derived from the low speed of the acoustic channel, only $1500 \mathrm{~m} / \mathrm{s}$, affects the time spent for a transmission process between two nodes, especially in a four handshake process (RTS/CTS/DATA/ACK). In

other words, doubling the contention window at every collision opportunity may be too much of an increase since a large contention window will make the node wait for a very long time. Then the backoff time does not need to be as big as in wireless to minimize the collisions during contention among multiple nodes.

A very simple modification of the BEB algorithm that uses a factor of 1.25 instead of 2 to reduce the contention window is introduced. The 1.25 value presented in the proposed 


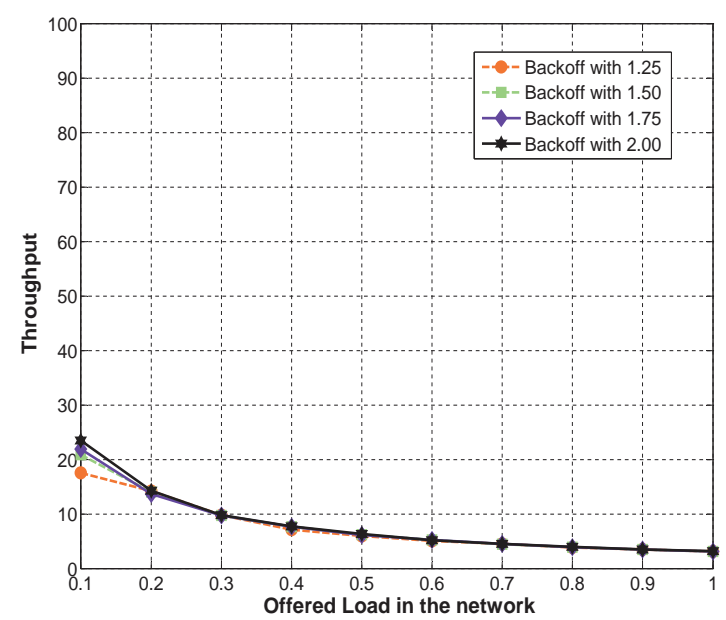

Figure 3.10: Different backoffs using packet size $=150$ bytes and $B E R=1 \times 10^{-3}$.

backoff algorithm is obtained by varying the initial factor 2 in the simulations, and analyzing the throughput behavior when the load in the network is increased. In Figure 3.10 and Figure 3.11 the throughput in an underwater network for the proposed data link protocol is shown, using different values for the backoff time. As seen, the throughputs are similar despite the size of the backoff interval. The reason is that in $2 \mathrm{MAC}$, collisions happen with a very low probability because nodes can access the channel simultaneously, and therefore a minimum number of backoffs will appear.

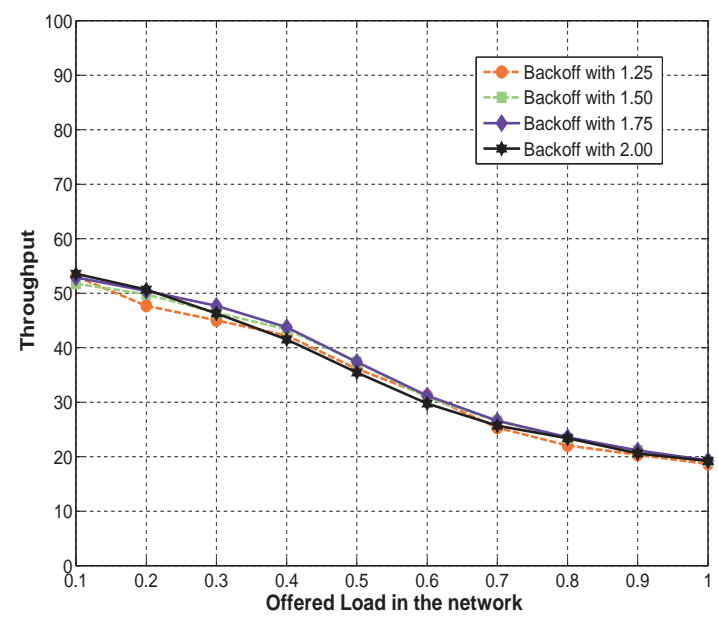

Figure 3.11: Different backoffs using packet size $=300$ bytes and $B E R=1 \times 10^{-4}$. 
The proposed backoff algorithm is also compared with other traditional backoff algorithms to verify the advantage of using the proposed one. It is compared with the one presented in [43], which increases the contention window by a factor of 1.75 . The algorithm, which is depicted in Equation 3.2, increases the contention window by a factor of 1.25 and limits the number of increments to a small number, depending on the minimum and maximum congestion window values.

For the case that the $C W_{M i n}$ is equal to 4 , and the $C W_{M a x}$ is equal to 9 , the number of increments is 3 . Once the retransmitted packet goes through, the contention window is not reset to $C W_{\text {Min }}$, as in 802.11 , but rather is reduced by that value for every successful packet. Given the numbers for $C W_{\text {Min }}$ and $C W_{\text {Max }}$ utilized for example, the contention window goes to $C W_{M i n}$ in just two steps.

$$
\begin{cases}C W=\min \left(1.25 C W, C W_{\text {Max }}\right) & \text { when collisions occur } \\ C W=\max \left(C W-C W_{\text {Min }}, C W_{\text {Min }}\right) & \text { otherwise }\end{cases}
$$

Figure 3.12 shows the throughput obtained by simulations of the three schemes as a function of the offered load using packets of 300 bytes, an underwater channel error model derived from [45], and the combined SW-MER/2MAC data link layer.

As it can be seen, the proposed algorithm improves the throughput especially at low network loads, due to $2 \mathrm{MAC}$ considerably reducing the number of collisions present in the channel. Looking at the figure, since the performance of the three algorithms is similar at higher loads, one may think that the protocol is not working well when it is supposed to provide more benefits; however, this is not true. What happens is that at high loads the performance is dominated by the bad underwater channel, mostly by retransmissions of packets in error due to the channel, not due to collisions. 


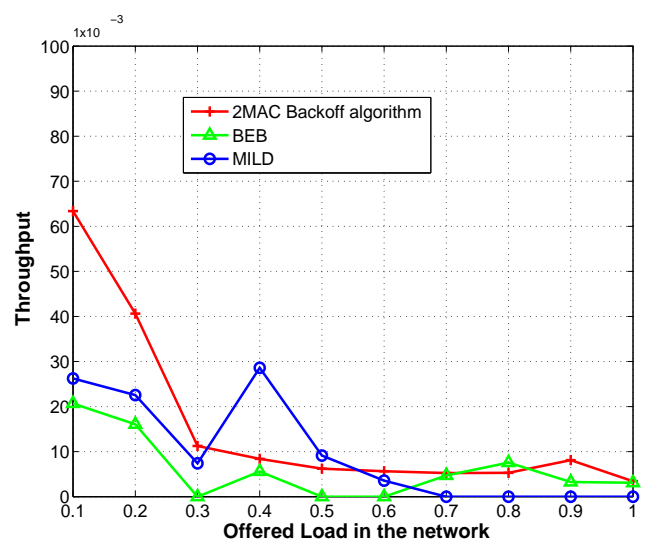

Figure 3.12: Throughput with different backoff algorithms. 


\section{Chapter 4: SW-MER: A Stop and Wait Window-Based Logical Link Control Protocol With Exponential Retransmissions}

The underwater communication channel is very noisy, and the bit error rate (BER) also changes depending on the depth and other factors, such as salinity. Normally the errors are correlated, like in wireless communications, but fading is deeper and longer under water producing more and burstier errors. Further, as in wireless communications, transducers for acoustic communications are half duplex, negating the possibility of using more efficient flow and congestion control mechanisms at the logical link control layer. As a result, most data link layers use the simple but inefficient stop and wait protocol instead of a sliding window-based approach at the logical control sublayer.

This chapter introduces a new logical link control protocol that uses a window stop and wait protocol, and multiple copies of packets are sent in exponential retransmissions named SW-MER. The proposed SW-MER protocol includes an exponential retransmission strategy that provides a highly reliable service over high error prone channels and a stop and wait window-based mechanism to increase the channel utilization over long propagation delay channels. SW-MER is described in detail and comparisons with other traditional logical link control protocols are shown. 


\subsection{SW-MER Description}

The SW-MER protocol is a combination of stop and wait and sliding window protocols. The transmitter sends a group of $M$ packets (window size of the transmission) and then waits for the (group) acknowledgment. As in the regular stop and wait protocol, the sender is not allowed to send more packets until the acknowledgment is received. In order to implement the SW-MER protocol, each packet contains additional information, such as a consecutive number representing its position in the window, a number that tells how many packets are missing to finish the reception of the current window, and the number of times the packet is being repeated in that window.

At the other end, the receiver verifies that the incoming packets are error-free and in sequence. If so, it sends them to the upper layer and acknowledges them all in one ACK packet to the sender. This ACK packet contains a vector $V$ of size $M$ in which every position in the vector reflects the state of every packet received, as follows:

$$
V[i]=\left\{\begin{array}{cl}
1 & \text { if packet } i \text { arrived with errors } \\
& \text { or did not arrive } \\
0 & \text { if packet } i \text { arrived without errors }
\end{array}\right.
$$

Upon receiving the ACK with vector $V[i]$, the sender retransmits all packets $i$ that were not received correctly. As in any ARQ protocol, the SW-MER sender also waits for the corresponding ACK packet before a retransmission timer expires. If a timeout occurs, the sender retransmits the same $M$ packets that were sent in the last window; otherwise, only those packets with $V[i]=1$ are retransmitted, which are sent in the initial positions of the following window. If there is enough space in the next window after including the 




Figure 4.1: First transmission, $M=6$ and packets 2 and 3 arrive with errors.

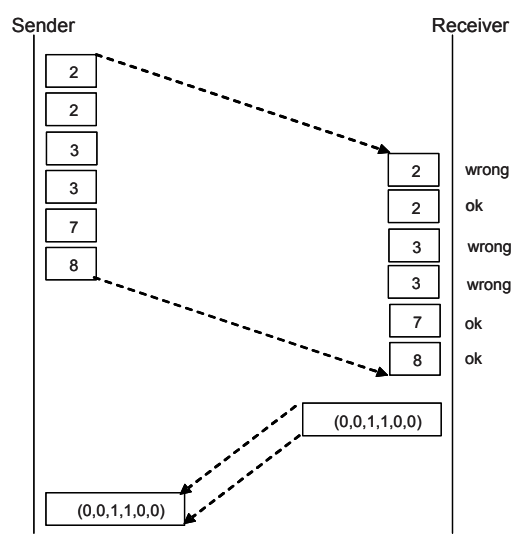

Figure 4.2: Second transmission, $M=6$ and packet 3 arrive with errors again.

retransmitted packets, the sender fills the rest of the window with new packets. In the case of retransmissions of already retransmitted packets, the sender repeats those packets in an exponential manner, i.e., packet $i$ will be retransmitted $2^{n}$ times in the following window until a maximum of $M$ times, where $n$ is the number of windows where packet $i$ has been received with errors. Figures 4.1 and 4.2 show graphically how the window mechanism of the SW-MER protocol works in the case of a sender that wants to send 8 packets using a window of size $M=6$.

After the first transmission, the receiver sends an ACK packet with vector $V$ saying that packets 2 and 3 arrived with errors (as seen in Figure 4.1). Upon the reception of the ACK 
packet, the sender transmits packets 2 and 3 two times each, and fills the rest of the window with new packets. During this second transmission, packet 3 arrives with errors again (none of the two copies arrived without errors). The receiver sends the appropriate ACK packet with the new vector $V$ (as seen in Figure 4.2). Then, the sender retransmits packet $32^{2}$ times in the next window. The process continues until the packet is received correctly or the maximum number of retransmissions is reached, which is set in our protocol to the window size $M=6$. With this procedure, more copies of the same packet are transmitted in case of repeated errors with the idea of increasing the reliability of the protocol.

An important aspect of the protocol is the size of the buffer at the receiver. If the buffer is not dimensioned appropriately, packets might be dropped because of lack of space. In order to guarantee that packets are sent to the upper layer in order, packets that arrive correctly are stored in memory and kept there until any of the missing packets are retransmitted and received correctly. Given the size of the window, $M$, and taking into account that in each retransmission copies of wrong packets are increased exponentially, the size of the buffer $B S$ needed to guarantee the operation of the protocol is given by Equation 4.2 as:

$$
B S=M \times\left(\log _{2} M-1\right)+2
$$

To deduce this formula, the worst case can be analyzed by having only one packet retransmitted until its amount of copies gets the window size $M$, using the following example: with $M=8$, the first time 8 new packets will be sent, the second time 2 copies of the packet arrived with errors are retransmitted including 6 new packets, the third time 4 copies are sent including 4 new packets, and the last retransmission will only have copies of the packet arrived with errors. In total, at the receiver 18 different packets arrive that 
need to be temporarily saved in the buffer. Then the buffer size should be $B S=8 \times\left(\log _{2} 8-1\right)+$ $2=18$.

\subsection{SW-MER State Transitions}

The proposed logical link layer protocol has the following states: Selecting Possible Packets, Updating Copies of each Packet in the Window, Listen, Waiting for CTS, Sending Window of Packets, Waiting for ACK Vector, Identifying Packets Sent with Errors, Receiving RTS, Preparing the CTS to be Sent, Waiting to Receive Packets, Identifying which Packets did not Arrive, Checking Packets, Enqueuing Packets without Errors, Verifying Packets to be Sent to the Upper Layer, and Generating the ACK Vector. Figures 4.3 and 4.4 show the sender and receiver state machines for the new protocol, respectively. The states and state transitions needed in the sender and receiver process at the logical link control layer are explained next.

\subsubsection{Selecting Possible Packets}

Once the node has packets to transmit, some of them are selected from the corresponding queue that has all the packets generated for its application layer. The amount of packets selected depends on the number of packets defined for the window transmission, and will be the same for each channel. This number is a constant assigned at the beginning of the entire transmission, and is the same value for all the nodes in the network. These packets are saved temporarily in two buffers, one for each channel, until it is decided in the next state which of them will be sent. 


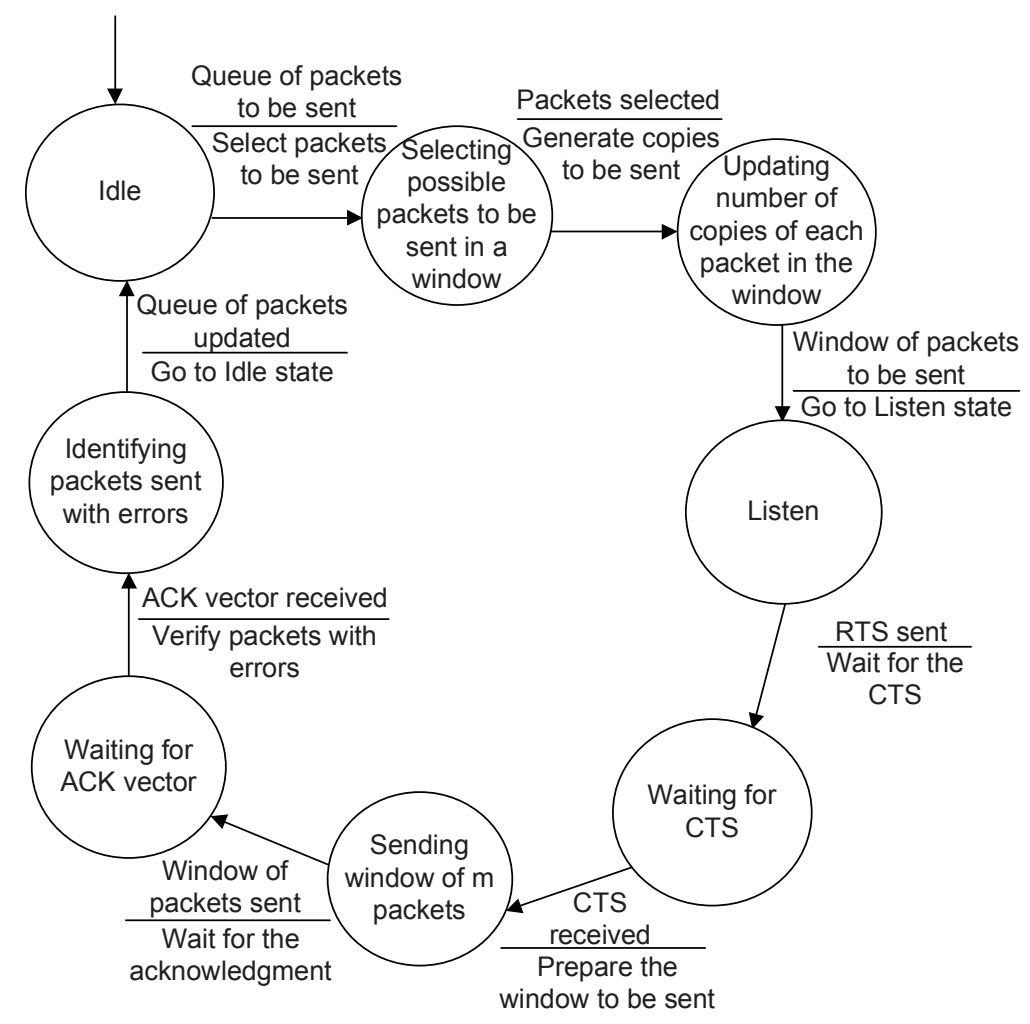

Figure 4.3: Finite state machine of the process in the sender at the LLC layer.

\subsubsection{Updating Copies of Each Packet in the Window}

For each packet, the transmitter keeps a value that represents the amount of copies to be sent in each window until either the packet is accepted or the maximum number of trials is reached. The window size in each transmission is $m$, and each window will group not only repeated packets but also new ones, if there is enough space to do it.

The first time a packet is sent, only one copy is transmitted. In later retransmissions of the same packet, the amount of copies to be sent is increased exponentially by two. This value will increase until the amount of packets to be transmitted in the window is reached, and once this happens, the same amount of copies will continue being sent until the max- 


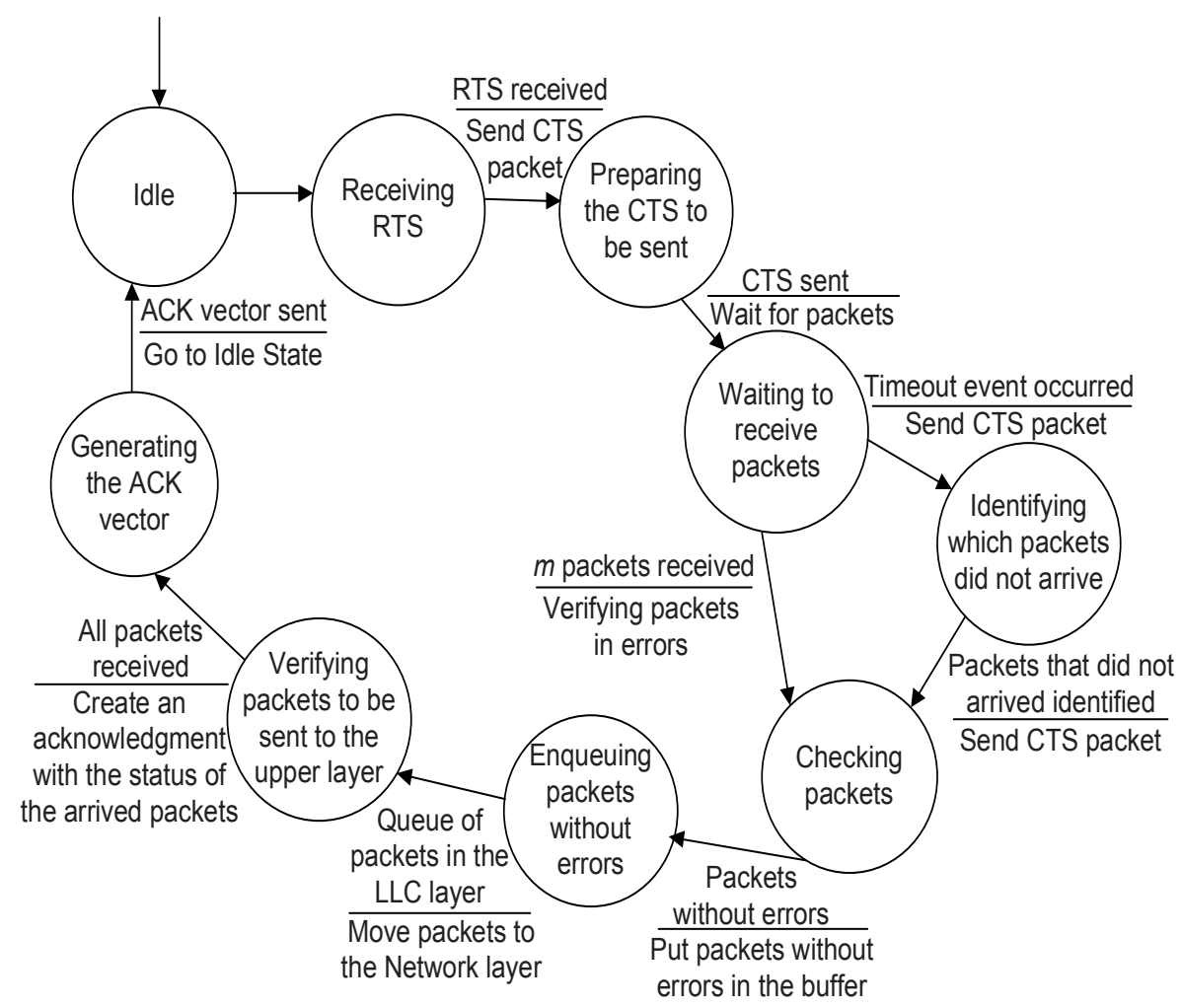

Figure 4.4: Finite state machine of the process in the receiver at the LLC layer.

imum number of trials is accomplished. Increasing the amount of copies for packet retransmissions will improve the probability of a packet to arrive without errors in the receiver and as a consequence, increases the reliability of the channel that is the main goal with this proposed protocol. Figure 4.1 and 4.2 show how the exponential retransmission works, in order to get a more reliable channel.

\subsubsection{Listen}

After the window of packets to be transmitted is created, the node starts a listen process to know whether or not other nodes are also transmitting in order to avoid collisions. 
Then, an RTS is sent in which is indicated the total time needed for sending windows of $m$ packets to the receiver.

\subsubsection{Waiting for CTS}

The transmitter waits for a certain amount of time, defined as a CTS timeout, to receive the CTS from both receivers. If the timeout is reached due to at least one CTS is not received, the node will again initiate a transmission process with the receiver; otherwise, the CTS is received in which the receiver is informing that the process to start a data transmission has been accepted either for one node or both.

\subsubsection{Sending Window of Packets}

In this state, the transmitter starts to send the window of $m$ packets to both receivers. When the data transmission finishes, the transmitter will go the next state to receive the corresponding acknowledgments.

\subsubsection{Waiting for ACK Vector}

The node stays in this state until at least one or both ACK vectors are received, otherwise a timeout is accomplished and the node will have to start the entire transmission process again. 


\subsubsection{Identifying Packets Sent With Errors}

After at least one ACK vector arrives to the sender, the vector is reviewed to identify which packets arrived with and without errors. Packets that arrived without errors are deleted from the sender queue; the others are kept until a maximum number of trials is achieved or they are accepted.

\subsubsection{Receiving RTS}

Because every node has two channels, one per each neighbor, it can receive RTS control packets from either one or both channels. Once the receiver starts to accept an RTS, it will wait for a short time to receive the RTS from the other neighbor. At the end of the time, the receiver will go to the next state to answer the sender.

\subsubsection{Preparing the CTS to be Sent}

After the RTS control packets arrive, either one or two with their corresponding time for each window of data packets, the receiver will save the most time between both RTS. Finally, the CTS packet is sent to the transmitter, either one or two, as an acceptance of a transmission. 


\subsubsection{Waiting to Receive Packets}

In this state, the receiver waits until either each window of packets arrives, or a timeout occurs because at least one of the packets never arrives in the corresponding window. If the timeout happens, the receiver is able to identify which packets did not come in every window because every packet sent by the transmitter has two additional fields. The first one represents the position of the packet in the window, and the second one represents the ordinal copy number.

\subsubsection{Checking Packets}

The receiver verifies whether the packets arrived with or without errors by applying a well-known Cyclic Redundancy Check (CRC) function, classifying packets into those that arrived with and without errors.

\subsubsection{Enqueuing Packets without Errors}

Packets arriving without errors are temporarily enqueued in the logical link layer until a consecutive number of $m$ packets arrive without errors, and packets arriving with errors are eliminated. The queue has a maximum number of packets to be enqueued. After that value, every packet that arrives without errors will be dropped, and the corresponding information will be lost. 


\subsubsection{Verifying Packets to be Sent}

Once a consecutive number of $m$ packets is achieved in the queue, they are sent to the upper layer and deleted from the queue. The purpose of sending the packets to the upper layer is to verify whether those packets belong to that node as a final destination, or whether they must be retransmitted to the next neighbor.

\subsubsection{Generating the ACK Vector}

In this state, the receiver is in charge of creating an ACK control packet to tell the transmitter the state of each packet received. This ACK is a vector in which each position will represent a value of either 0 or 1 , where 0 means that the packet arrived without errors, and 1 means that the packets arrived with errors or did not arrive. At the end, the ACK vector is sent to the transmitter. 


\section{Chapter 5: Analytical Models for the Proposed Data Link Layer Protocols}

For both 802.11 wireless local area networks and underwater acoustic networks, the MAC layer has a relevant role in terms of the throughput and the reliability of the network. Throughput, for example, has been a fundamental area of research to obtain a more efficient MAC layer.

Several models have been presented in the literature to represent the throughput in saturated load conditions for 802.11 networks, but not for underwater networks, in which [46] is a fundamental one. In [46] the model works with the assumption of having only collisions in the network and there is an error-free channel condition. In that research a two-dimensional Markov chain is proposed to model the backoff process and the throughput is described in terms of that backoff. In [47], the throughput is derived from [46] with the difference that the channel is not error-free. It is assumed that control packets such as RTS, CTS and ACK are very small and they will never be corrupted by the channel; as a consequence only data packets will be affected by the wireless medium. In [48], the throughput is described as a function of both collisions and error-prone channels, but for a MAC protocol in which only data and acknowledgment packets are involved in a transmission process in wireless networks.

In this chapter, an analytical model is presented to obtain the throughput for the proposed data link layer protocol for underwater networks. This analytical model is based on the 
discrete Markov model presented in [46] and [48]. The difference starts from the MAC protocol where the saturation throughput is obtained. [46] and [48] model the traditional 802.11 MAC protocol that uses one channel per node to communicate with the others, and here [9] is the protocol that has been modeled in which each node presents two channels for the communication. Another difference is that for the saturation throughput, 2MAC supposes that RTS, CTS, and ACK control packets, and also data packets can be corrupted by the error-prone channel.

In the next section, the description of the analytical model for the proposed data link protocol will be divided into 2MAC and SW-MER. In the first section, the analytical model for $2 \mathrm{MAC}$ is presented and in the second section, the analytical model for SWMER is introduced.

\subsection{Analytical Model for 2MAC}

An analytical model for $2 \mathrm{MAC}$ is developed to calculate the throughput of the protocol, assuming an ideal channel condition, a finite number of nodes in the network, and the exponential backoff process modeled as a Markov chain. For the model design, it is also assumed a constant and independent collision probability of a packet transmitted in each window transmission for every node in the network, regardless of the amount of retransmissions.

The remainder of this section is organized as follows. Section 5.1.1 includes a brief description of the RTS/CTS transmission process of the 2MAC protocol. Section 5.1.2 describes the throughput analysis, and Section 5.1.3 presents the validation of the analytical model. 


\subsubsection{MAC RTS/CTS Transmission Process}

As explained before, the mechanism to access the channel implemented in 2MAC is also a Carrier Sense Multiple Access with Collision Avoidance (CSMA/CA) with RTS/CTS control packets, similar to the one designed for IEEE 802.11 protocols. However, 2MAC utilizes five type of frames, these are Request to Send (RTS), Clear to Send (CTS), Data, Acknowledgment (ACK) and Blocked to Send (BTS), as shown in Figure 3.7. The BTS frame is necessary because $2 \mathrm{MAC}$ nodes use two different channels, one for each neighbor. If there is a node $\mathrm{A}$ with two neighbors $\mathrm{B}$ and $\mathrm{C}$ and it decides to transmit data only to $\mathrm{B}, \mathrm{C}$ does not know what happens between $\mathrm{A}$ and $\mathrm{B}$ because it is listening in a different channel. In this case, $\mathrm{C}$ receives a BTS frame instead, with the time that $\mathrm{C}$ has to delay its transmission.

In addition to the frames, five types of timing intervals have been implemented. Three of them, the Short Interframe Space (SIFS), the Slot Time (st), and the Distributed Interframe Space (DIFS), are the same used in IEEE 802.11. The other two are Adjusted Response time (ARS) and Channel Assignment Time (CHA). ARS time is used for a node to wait for signals from both neighbors. Once the node receives a frame from one of its neighbors, it will wait for an ARS time to find out if another frame is coming from the other neighbor. Nodes wait for a CHA a short time before starting their transmissions, to identify whether both channels are ready or not to send packets and also to avoid collisions.

As explained in chapter 3, when a node wants to transmit either one window or two windows of packets, the channels are activated in a certain period of time called CHA. Once activated, they are monitored. If the channels are idle for a period of time called DIFS, the 
node generates a random backoff interval before the transmission of the RTS starts. This random backoff interval is named Contention period and is meant to reduce packet collisions. Otherwise, the backoff algorithm is triggered after the current transmission time finishes plus more DIFS and CHA times, as shown in Figure 3.7. Once the destination receives an RTS, it spends a period of time ARS to verify if there is another RTS coming from the other neighbor and another SIFS. Then, the CTS is sent to the transmitter. After the transmitter receives the CTS, it waits for an ARS plus a SIFS, and sends the data. The receiver transmits the corresponding ACK vectors once the data is received, and after an ARS plus SIFS.

The backoff algorithm adopted is an exponential backoff scheme, similar to the one used in 802.11. The time is divided in slots, $s t$, the backoff time is uniformly chosen in an interval $(0, W-1)$, and $W$ is the contention window that will be exponentially increased every time a retransmission fails. At the first transmission, $W$ starts at $C W_{\min }$, which is defined as the minimum contention window. Every time a retransmission fails, $W$ is increased as $W=1.25 W$ up to a maximum value $C W_{\max }$, where $C W_{\max }=1.25^{m} C W_{\min }$ and $m$ is the maximum number of times the backoff will be executed, named as the maximum backoff stage. Every time the channel is sensed idle, the backoff time is decremented in one slot time, otherwise when a transmission is detected then the backoff time is frozen and reactivated once the channel is idle again. The node transmits only when the backoff slot time reaches the 0 value. 


\subsubsection{Throughput Analysis}

The analytical model for the throughput is solved in a similar way like in [46]. One of the differences is that in [46] problems such as hidden and exposed terminal are not solved, an ideal channel is assumed. In 2MAC, hidden and exposed terminal problems do not exist because the protocol works with two channels in each node, one per neighbor as explained before, solving the possibility of occurring these problems.

To obtain the throughput analysis, a fixed number of nodes where each node always has a packet available to transmit, is assumed. As a consequence, a non-empty transmission queue of each node is assumed all the time.

In the next section, the backoff process is presented by means of a Markov chain model. Based on the Markov chain, the probability $\tau$ for a node to be transmitting in a transmission period is calculated. At the end, the throughput is obtained as a function of $\tau$.

\subsubsection{Packet Transmission Probability}

To obtain the packet transmission probability, $n$ nodes are assumed in the network, and an integer time scale is adopted where $t$ and $t+1$ represent consecutive slot times st. Each node has a packet available to transmit after a successful transmission, and waits for a random backoff time generated and saved in a backoff time counter. In each slot time the channel is sensed. If the channel is busy then the backoff time counter stops, otherwise it is decremented until it reaches 0 . As explained before, the contention window $W$ starts at $C W_{\min }$ and increases until it reaches the maximum contention window $C W_{\max }$, where $C W_{\max }=1.25^{m} W ; m$ represents the maximum backoff stage, and $W_{i}=1.25^{i} W$, such 


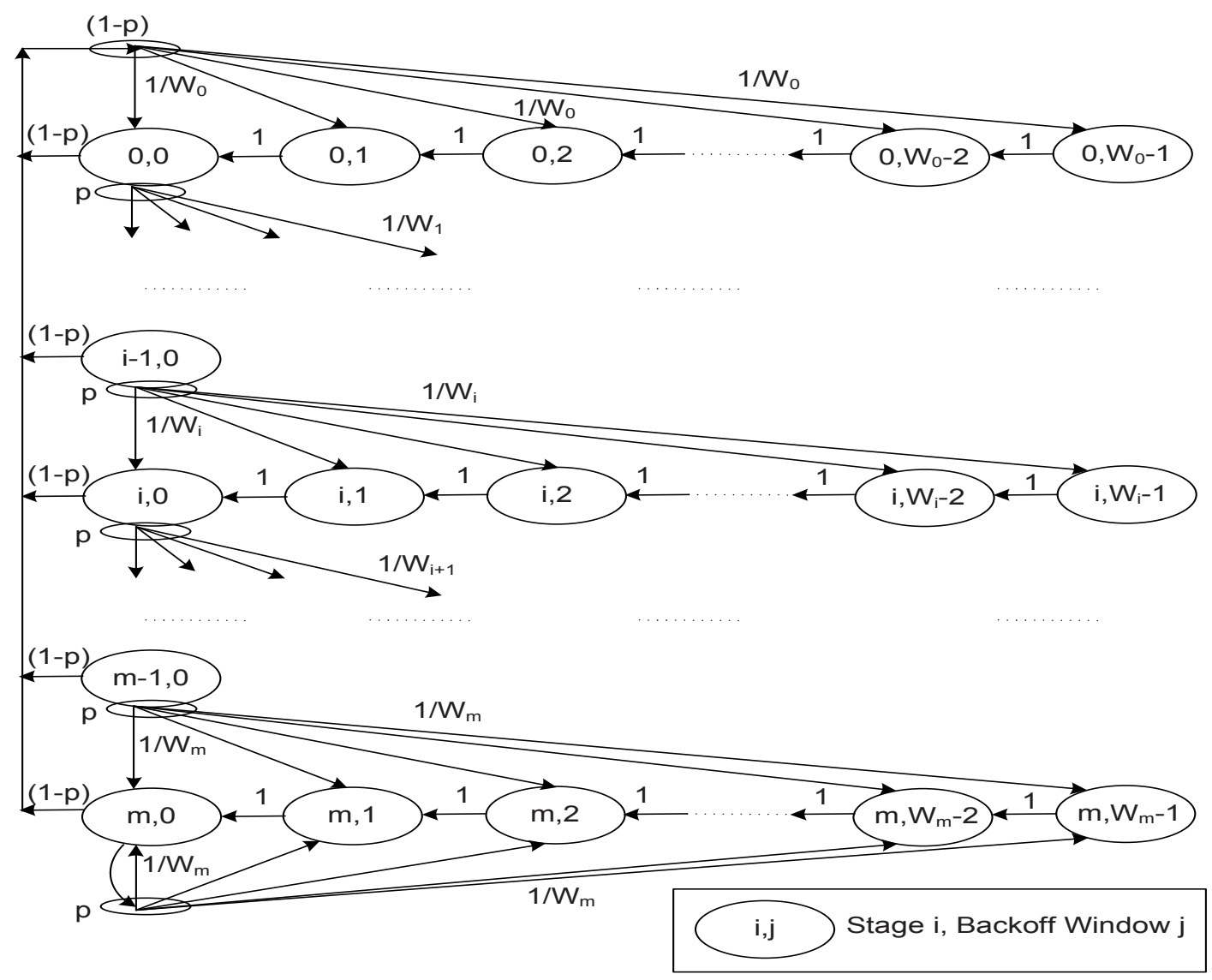

Figure 5.1: Markov chain for the backoff process [46].

that $i \in(0, m)$. In each retransmission attempt, a packet can collide with a constant and independent probability $p$, this $p$ is also defined in [46] as the transition probability from one stage to another. Like in [46], the backoff process is modeled with a Markov chain in which $\pi_{i j}$ represents the steady state probability for a backoff stage $i$ and backoff counter $j$, as shown in Figure 5.1.

Following [46], in the Markov chain every $\left(i, W_{i}\right)$ represents a bidimensional state where the first parameter represents the backoff stage $\mathrm{i} \in(0, m)$ and the second parameter is the backoff counter $\left(0, W_{i}\right)$ where $\mathrm{i} \in(0, m)$. The non-null one-step probabilities derived in [46] from the Markov chain are: 


$$
\begin{array}{cc}
P\{(i, k) \mid(i, k+1)\}=1, & k \in\left(0, W_{i}-2\right), \\
P\{(0, k) \mid(i, 0)\}=(1-p) / W_{0}, & k \in\left(0, W_{0}-1\right), \quad i \in(0, m) \\
P\{(i, k) \mid(i-1,0)\}=p / W_{i}, & k \in\left(0, W_{i}-1\right), \quad i \in(1, m) \\
P\{(m, k) \mid(m, 0)\}=p / W_{m}, & k \in\left(0, W_{m}-1\right)
\end{array}
$$

These probabilities represent the conditional collision probabilities as explained in [46] in which $(\mathrm{i}, \mathrm{j})$ represents the backoff stage $i$ with the backoff time counter $j$. The equation 5.1 means that at the beginning of each slot time the backoff time counter is decremented with probability of 1. The probability in Equation 5.2 indicates that after a successful transmission, a new packet to be transmitted requires that the transmitter starts with its backoff stage 0 . The corresponding backoff time counter will be initialized with a number uniformly chosen in the range $\left(0, W_{0}-1\right)$.

The probability in Equation 5.3 represents when an unsuccessful transmission occurs at the backoff stage $i-1$, then the backoff state increases and the new backoff time counter is initialized in a value uniformly selected from $\left(0, W_{i}\right)$. Finally, Equation 5.4 means that once the backoff stage reaches $m$, it will not be increased in a new packet transmission. It will stay there until the maximum number of trials defined by the protocol is reached; then the packet will be dropped. 
Applying the non-null one-step probabilities in the backoff model proposed in 2MAC [9], the equations are derived as follows:

$$
\begin{aligned}
& P\{i, k \mid i, k+1\}=1, \quad k \in\left(0, W_{i}-2\right), \quad i \in(0, m) \\
& P\{0, k \mid i, 0\}=\frac{1-p}{W}, \quad k \in(0, W-1), \quad i \in(0, m) \\
& P\{i, k \mid i-1,0\}=\frac{p}{1.25^{i} W}, \quad k \in\left(0,1.25^{i} W-1\right), \quad i \in(1, m) \\
& P\{m, k \mid m, 0\}=\frac{p}{1.25^{m} W}, \quad k \in\left(0,1.25^{m} W-1\right)
\end{aligned}
$$

The steady state probabilities derived in [46] for the Markov chain represented by $\pi_{i, j}$, for a backoff stage $i$ and a backoff counter $j$, are the following:

$$
\begin{array}{rr}
\pi_{i, 0}=p \cdot \pi_{i-1,0} & 0<i<m \\
\pi_{m-1,0} \cdot p=\pi_{m, 0} & \Rightarrow \pi_{i, 0}=p^{i} \cdot \pi_{0,0}
\end{array}
$$




$$
\begin{gathered}
\pi_{0,0}=(1-p) \cdot \sum_{j=0}^{m} \pi_{j, 0} \\
\tau=\sum_{j=0}^{m} \pi_{j, 0}
\end{gathered}
$$

The only possibility to enter the set $\left\{(0,0),(0,1), \ldots,\left(0, W_{0}-1\right)\right\}$ is after a successful window packet transmission from $\{(0,0),(1,0),(2,0) \ldots,(m, 0)\}$. The successful transmission happens with the probability shown in Equation 5.13.

$$
(1-p) \sum_{j=0}^{m} \pi_{j, 0}
$$

A counter value is chosen followed in the range $\left\{0,1, \ldots, k, \ldots, W_{0}-1\right\}$, which happens with probability $\frac{W_{0}-k}{W_{0}}$. Then, having $k \in\left(1, W_{i}-1\right)$ all the steady state probabilities are obtained as follows:

$$
\pi_{i, k}=\frac{W_{i}-k}{W_{i}} \cdot \begin{cases}(1-p) \sum_{j=0}^{m} \pi_{j, 0}, & \text { if } i=0 \\ p \cdot \pi_{i-1,0}, & \text { if } 0<i<m \\ p \cdot\left(\pi_{m-1,0}+\pi_{m, 0}\right), & \text { if } i=m\end{cases}
$$


Based on Equations 5.10 and 5.11, Equation 5.14 can be modified and the steady state probability can be obtained as follows:

$$
\begin{aligned}
& \pi_{i, k}=\frac{W_{i}-k}{W_{i}} \cdot \begin{cases}\pi_{0,0}, & \text { if } i=0 ; \\
\pi_{i, 0}, & \text { if } 0<i<m ; \\
\pi_{m, 0}, & \text { if } i=m\end{cases} \\
& \Rightarrow \pi_{i, k}=\frac{W_{i}-k}{W_{i}} \cdot \pi_{i, 0}, \quad i \in(0, m), k \in\left(0, W_{i}-1\right)
\end{aligned}
$$

Using the normalization condition that the sum of all steady state probabilities should be equal to 1 , the following equation is obtained:

$$
\begin{array}{r}
1=\sum_{i=0}^{m} \sum_{j=0}^{W_{i}-1} \pi_{i, j}=\sum_{i=0}^{m} \pi_{i, 0} \sum_{j=0}^{W_{i}-1} \frac{W_{i}-j}{W_{i}}=\sum_{i=0}^{m} \pi_{i, 0}\left[\sum_{j=0}^{W_{i}-1} 1-\frac{1}{W_{i}} \sum_{j=0}^{W_{i}-1} j\right]= \\
=\sum_{i=0}^{m} \pi_{i, 0}\left(\frac{W_{i}+1}{2}\right)=\frac{1}{2}\left[\sum_{i=0}^{m} \pi_{i, 0} \cdot W_{i}+\sum_{i=0}^{m} \pi_{i, 0}\right]=\frac{1}{2}\left[\sum_{i=0}^{m} \pi_{i, 0} \cdot W_{i}+\frac{\pi_{0,0}}{(1-p)}\right]= \\
=\frac{1}{2}\left[\sum_{i=0}^{m-1} \pi_{i, 0} \cdot W_{i}+\pi_{m, 0} \cdot W_{m}+\frac{\pi_{0,0}}{(1-p)}\right]=
\end{array}
$$


By replacing the backoff time defined in [9] that is $W_{i}=(1.25)^{i} \dot{W}=\left(\frac{5}{4}\right)^{i} \dot{W}$ in Equation 5.17, the stationary distribution of the Markov chain for a backoff stage 0 and a backoff counter 0 for $2 \mathrm{MAC}$ is obtained.

$$
\begin{gathered}
1=\frac{\pi_{0,0}}{2}\left[\sum_{i=0}^{m-1} p^{i}\left(\frac{5}{4}\right)^{i} \cdot W+\frac{p^{m}}{(1-p)}\left(\frac{5}{4}\right)^{m} \cdot W+\frac{1}{(1-p)}\right] \\
\Rightarrow \pi_{0,0}=\frac{8 \cdot\left(1-\left(\frac{5}{4} p\right)\right)(1-p)}{4 \cdot(W+1)\left(1-\left(\frac{5}{4} p\right)\right)+W p\left(1-\left(\frac{5}{4} p\right)^{m}\right)}
\end{gathered}
$$

The process to obtain the Equation 5.19 from Equation 5.18 is described in Appendix A.

The packet transmission probability or the probability $\tau$ that a node transmits in a randomly chosen transmission period displayed in Equation 5.12 for $2 \mathrm{MAC}$, can be represented in terms of $p$ and $W$.

$$
\begin{array}{r}
\tau=\frac{\pi_{0,0}}{(1-p)}=\frac{8 \cdot\left(1-\left(\frac{5}{4} p\right)\right)}{4 \cdot(W+1)\left(1-\left(\frac{5}{4} p\right)\right)+W p\left(1-\left(\frac{5}{4} p\right)^{m}\right)}= \\
=\frac{8}{4 \cdot(W+1)+W p \cdot \frac{1-\left(\frac{5}{4} p\right)^{m}}{1-\left(\frac{5}{4} p\right)}} \\
\Rightarrow \tau(p)=\frac{8}{4 \cdot(W+1)+W p \cdot \frac{1-\left(\frac{5}{4} p\right)^{m}}{1-\left(\frac{5}{4} p\right)}}
\end{array}
$$

Now, it is necessary to verify if $\tau$ is continuous in the range $p \in(0,1)$. To do this, $\tau$ is evaluated in 0,1 and 4/5: 


$$
\begin{gathered}
\tau(0)=\frac{8}{4 \cdot(W+1)+0} \\
\Rightarrow \tau(0)=\frac{2}{(W+1)} \\
\tau(1)=\frac{8}{4 \cdot(W+1)+W \cdot \frac{1-\left(\frac{5}{4}\right)^{m}}{1-\left(\frac{5}{4}\right)}}
\end{gathered}
$$

To evaluate $\tau$ in 4/5, Equation 5.20 can be represented as follows:

$$
\begin{gathered}
\tau(p)=\frac{8}{4 \cdot(W+1)+W p \cdot \sum_{i=0}^{m-1}\left(\frac{5}{4} p\right)^{i}} \\
\Rightarrow \tau\left(\frac{4}{5}\right)=\frac{2}{(W+1)+W m\left(\frac{1}{5}\right)}
\end{gathered}
$$

Considering the results from Equations 5.21, 5.22 and 5.24, it is proved that $\tau$ is continuous in the range of $p$.

The value of $\tau$ is calculated in such a way that the probability $p$ is obtained as follows:

$$
p=1-\left(1-P_{c}\right) \cdot\left(1-p_{e}\right)
$$


The transmission error event generated due to errors in the channel $p_{e}$ is calculated in the following equation:

$$
\begin{gathered}
p_{e}=p_{e}^{r t s}+p_{e}^{c t s}+p_{e}^{d a t a}+p_{e}^{a c k}-p_{e}^{r t s} \cdot p_{e}^{c t s} \cdot p_{e}^{d a t a} \cdot p_{e}^{a c k} \\
P_{c}=1-(1-\tau)^{n-1}
\end{gathered}
$$

$1-P_{c}$ represents that there is no collision when a packet was transmitted and $1-P_{e}$ means that there are no bits with errors in a received packet. The descriptions of the variables can be seen in Table 5.1.

\subsubsection{Saturation Throughput}

Saturation throughput is defined as the ratio of successful data transmitted over a randomly chosen transmission period, as seen in Equation 5.28.

$$
T=\frac{\text { E[number of channels idle }] \cdot E[\text { Data transmitted in a transmission period }]}{\text { E[length of the transmission period }]}
$$

To obtain the saturation throughput like in [46], it is necessary to analyze what happens in a randomly chosen transmission period. There are seven different transmission period situations, as shown in Figure 5.2. To calculate these transmissions, it is assumed that the time required either to transmit a CTS, an RTS or an ACK control packet are the same and it is named as $T_{c t l}$. 


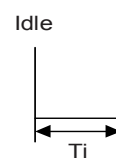

Success

\begin{tabular}{|c|c|c|c|c|c|c|c|c|c|c|c|}
\hline RTS & ARS & SIFS & CTS & ARS & SIFS & DATA & ARS & SIFS & ACK & $\mathrm{ARS}$ & DIFS \\
\hline
\end{tabular}


CTS error



DATA error
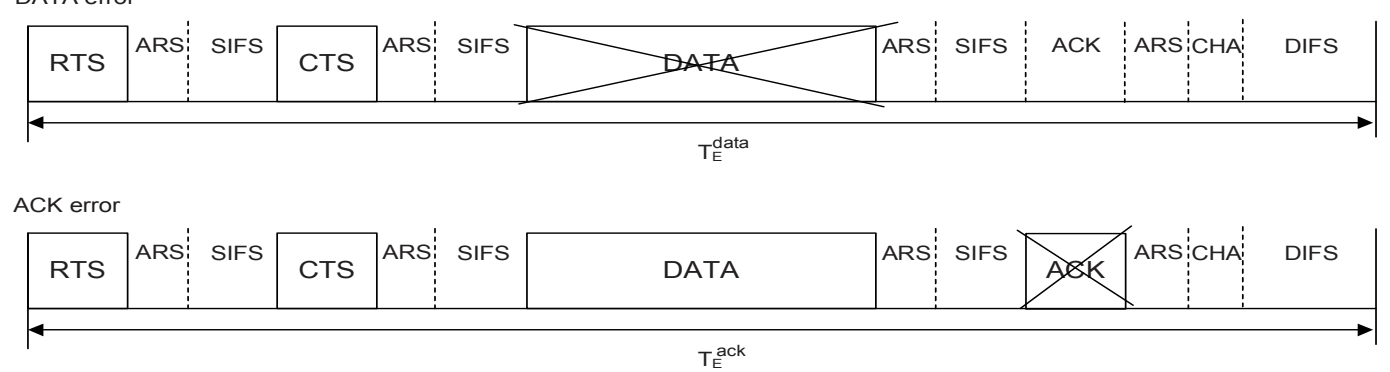

Figure 5.2: Transmission periods in an error-prone channel. 
Table 5.1: Notations used to obtain the saturation throughput.

\begin{tabular}{|c|c|c|c|}
\hline$T_{i}$ & The idle transmission period & $T_{\text {suc }}$ & $\begin{array}{l}\text { Transmission period in which the channel is sensed busy } \\
\text { because of a successful transmission }\end{array}$ \\
\hline RTS & Request To Send control packet & CTS & Clear To Send control packet \\
\hline ACK & Acknowledgment control packet & $\bar{T} \overline{c o l}$ & $\begin{array}{l}\text { Time that the channel is sensed busy because of a } \\
\text { collision }\end{array}$ \\
\hline$T_{E}^{r t s}$ & $\begin{array}{l}\text { Time that the channel is sensed busy because } \\
\text { of an RTS frame error transmission }\end{array}$ & $T_{E}^{c t s}$ & $\begin{array}{l}\text { Time that the channel is sensed busy because of an } \\
\text { RTS frame error transmission }\end{array}$ \\
\hline$T_{E}^{\text {data }}$ & $\begin{array}{l}\text { Time that the channel is sensed busy because } \\
\text { of a data frame error transmission }\end{array}$ & $T_{E}^{a c k}$ & $\begin{array}{l}\text { Time that the channel is sensed busy because of an } \\
\text { ACK frame error transmission }\end{array}$ \\
\hline$P P_{t r}$ & $\begin{array}{l}\text { Probability of having at least one node } \\
\text { transmitting in a transmission period }\end{array}$ & $p$ & $\begin{array}{l}\text { Transmission failure probability of a node either } \\
\text { for a collision or a transmission error event }\end{array}$ \\
\hline$P_{x}$ & $\begin{array}{l}\text { Probability that only one node transmits on the } \\
\text { channel }\end{array}$ & $P_{c}$ & $\begin{array}{l}\text { Probability of a collision seen by a packet being } \\
\text { transmitted on the channel }\end{array}$ \\
\hline$P_{\text {suc }}$ & $\begin{array}{l}\text { Probability of a successful transmission in a } \\
\text { transmission period }\end{array}$ & $\mathrm{H}$ & Packet header \\
\hline$T_{s}^{2 m a c}$ & $\begin{array}{l}\text { Average time that the channel is sensed busy } \\
\text { because of a successful transmission }\end{array}$ & $T_{c}^{2 m a c}$ & $\begin{array}{l}\text { Average time that the channel is sensed busy by each } \\
\text { node during a collision }\end{array}$ \\
\hline$E\left[P k t_{t r}\right]$ & $\begin{array}{l}\text { Average data size successfully transmitted in } \\
\text { a transmission period }\end{array}$ & $E\left[W_{p k t}\right]$ & Average window packet size to be transmitted \\
\hline$E\left[L_{s t}\right]$ & Average length of a transmission period & W & Number of packets to be transmitted in a window \\
\hline Pkt $t_{\text {size }}$ & Size of the data packet in bits & $\mathrm{n}$ & $\begin{array}{l}\text { Number of nodes in the network that can contend the } \\
\text { channel, each one transmitting with probability } \tau\end{array}$ \\
\hline$p_{e}^{\text {data }}$ & Data frame error probability & $p_{e}^{r t s}$ & RTS frame error probability \\
\hline$p_{e}^{c t s}$ & CTS frame error probability & $p_{e}^{a c k}$ & ACK frame error probability \\
\hline lrts & Size in bits of the RTS frame & lcts & Size in bits of the CTS frame \\
\hline ldata & Size in bits of the DATA frame & lack & Size in bits of the ACK frame \\
\hline$B E R$ & Bit error probability & $\alpha$ & Duration of an empty transmission period \\
\hline$T T_{r t s}$ & Duration of an RTS control packet transmission & $T T_{c t s}$ & Duration of a CTS control packet transmission \\
\hline$T_{t x d}$ & Duration of a DATA packet transmission & $T_{t x a}$ & Duration of an ACK control packet transmission \\
\hline ARS & Adjusted response time & CHA & Channel assignment duration \\
\hline$P_{E}^{r t s}$ & $\begin{array}{l}\text { Probability that a transmission error occurs } \\
\text { because the RTS frame is corrupted when only } \\
\text { one node is transmitting }\end{array}$ & $P_{E}^{c t s}$ & $\begin{array}{l}\text { Probability that an RTS frame is successfully transmi- } \\
\text { tted but the corresponding CTS frame is corrupted } \\
\text { because of transmission errors }\end{array}$ \\
\hline$P_{E}^{\text {data }}$ & $\begin{array}{l}\text { Probability that an RTS and CTS are successfully } \\
\text { transmitted but the corresponding data frame are } \\
\text { corrupted }\end{array}$ & $P_{E}^{a c k}$ & $\begin{array}{l}\text { Probability that an RTS, CTS and data frames is } \\
\text { successfully transmitted but the ACK frame is } \\
\text { corrupted due to transmission errors }\end{array}$ \\
\hline$T_{\text {prop }}$ & Propagation delay & $T_{c t l}$ & Duration of a control packet transmission \\
\hline$P_{\text {col }}$ & $\begin{array}{l}\text { Probability that at least two nodes start transmissions } \\
\text { in a same transmission period }\end{array}$ & & \\
\hline
\end{tabular}

The first case in the transmission period corresponds to the time the node is idle, since

there are no packets to transmit. The second case represents the time spent for a node to

transmit the data starting with the RTS transmission and finishing with the ACK recep-

tion. The third case identifies that there was a collision at the time an RTS was sent. The

fourth case represents that the RTS arrived with errors to the receiver because of errors in

the channel. 
The fifth case defines the transmission period generated when the CTS arrived with errors to the sender due to channel problems. The sixth case is the time required when the data arrived with errors to the receptor because of errors in the channel and the last case is the time spent when the ACK arrived with errors at the sender also due to errors in the channel.

The corresponding variables that represent these transmission periods are defined in Table 5.1, and they can be obtained as follows:

$$
\begin{gathered}
T_{c t l}=T_{t x a}=T_{c t s}=T_{r t s} \\
T_{s u c}=T_{t x d}+4\left(A R S+T_{\text {prop }}\right)+3\left(T_{c t l}+S I F S\right)+C H A+D I F S \\
T_{E}^{c t s}=2\left(T_{c t l}+A R S+T_{p r o p}+S I F S\right) \\
T_{c o l}=T_{c t l}+A R S+T_{p r o p}+S I F S \\
T_{E}^{r t s}=T_{c o l} \\
T_{E}^{a c k}=T_{E}^{\text {data }}=T_{\text {suc }}
\end{gathered}
$$


As a consequence of the situations presented in a transmission period, some other variables or parameters that affect the throughput have to be defined, following a similar strategy used for error-prone wireless networks in [48]. These variables are also shown in Table 5.1, and the corresponding equations needed to compute them are the following:

$$
P_{t r}=1-(1-\tau)^{n}
$$

in which $(1-\tau)^{n}$ is the probability of no transmission in a transmission period for the $n$ nodes in the network. The probability of $P_{t r}$ and $P_{x}$ are defined in Table 5.1, and are needed to know the probability $P_{c o l}$ that at least two nodes transmit in the same transmission period generating collisions. $P_{c o l}$ affects the throughput of the network because the collision of two nodes will increment the total time for those nodes to obtain a successful transmission.

$$
\begin{gathered}
P_{x}=n \tau(1-\tau)^{n-1} \\
P_{c o l}=1-(1-\tau)^{n}-n \tau(1-\tau)^{n-1}=P_{t r}-P_{x}
\end{gathered}
$$

The following equation also affects the throughput in the case that a transmission is successful, so it displays the probability that the successful transmission can occur.

$$
P_{s u c}=P_{x}\left(1-p_{e}^{r t s}\right)\left(1-p_{e}^{c t s}\right)\left(1-p_{e}^{d a t a}\right)\left(1-p_{e}^{a c k}\right)
$$


All of the probabilities included in a successful transmission probability are calculated based on $B E R$ as follows:

$$
\begin{gathered}
p_{e}^{r t s}=1-(1-B E R)^{l r t s} \\
p_{e}^{c t s}=1-(1-B E R)^{l c t s} \\
p_{e}^{\text {data }}=1-(1-B E R)^{l d a t a}
\end{gathered}
$$

The data frame size is defined as ldata $=H+P k t_{\text {size }}$.

$$
p_{e}^{a c k}=1-(1-B E R)^{l a c k}
$$

To obtain the throughput, the average data size successfully transmitted should be known. This average depends on the average window packet size that must be submitted and is calculated as follows:

$$
E\left[W_{p k t}\right]=w \cdot P k t_{\text {size }}
$$


Then, the average data size successfully transmitted that depends on the average window packet size is obtained in the following equation.

$$
E\left[P k t_{t r}\right]=E\left[W_{p k t}\right] \cdot P_{s u c}
$$

The expected value of the length of the transmission period where $\left(1-P_{t r}\right)$ is the probability of no transmission in a transmission period (idle transmission period), can be calculated as follows:

$$
\begin{aligned}
& E\left[L_{s t}\right]=\left(1-P_{t r}\right) \alpha+T_{s u c} P_{s u c}+P_{c o l} \cdot T_{c o l}+T_{E}^{\text {data }} P_{E}^{\text {data }}+T_{E}^{a c k} P_{E}^{a c k}+ \\
& +T_{E}^{r t s} P_{E}^{r t s}+T_{E}^{c t s} P_{E}^{c t s} \\
& P_{E}^{r t s}=P_{x} p_{e}^{r t s} \\
& P_{E}^{c t s}=P_{x}\left(1-p_{e}^{r t s}\right) p_{e}^{c t s} \\
& P_{E}^{d a t a}=P_{x}\left(1-p_{e}^{r t s}\right)\left(1-p_{e}^{c t s}\right) p_{e}^{\text {data }} \\
& P_{E}^{a c k}=P_{x}\left(1-p_{e}^{r t s}\right)\left(1-p_{e}^{c t s}\right)\left(1-p_{e}^{\text {data }}\right) p_{e}^{a c k}
\end{aligned}
$$


Every node in $2 \mathrm{MAC}$ has two channels, one per neighbor, and to start a transmission it is only necessary to have at least one channel idle. Then, the number of channels expected to be idle are:

$\mathrm{E}[$ number of channels idle $]=$ number of channels $\cdot \mathrm{P}[$ at least one channel is idle $]=$

$$
=2 \cdot(.25+.25+.25)=1.5
$$

Then, the saturation throughput $T$ is calculated as follows:

$$
\begin{gathered}
T=\frac{\text { E[number of channels idle }] \cdot \mathrm{E}[\text { Data transmitted in a transmission period }]}{\text { E[length of the transmission period }]}=\frac{1.5 \cdot E\left[P k t_{t r}\right]}{E\left[L_{s t}\right]} \\
T=\frac{1.5 \cdot E\left[W_{p k t}\right] \cdot P_{s u c}}{\left(1-P_{t r}\right) \alpha+T_{s u c} P_{s u c}+P_{c o l} \cdot T_{c o l}+T_{E}^{\text {data }} P_{E}^{\text {data }}+T_{E}^{\text {ack }} P_{E}^{a c k}+T_{E}^{\text {rts }} P_{E}^{\text {rts }}+T_{E}^{c t s} P_{E}^{c t s}}
\end{gathered}
$$

\subsubsection{Model Validation}

Results for the throughput in the analytical model have been compared with the ones obtained by running the simulations. These results are based on the parameters included in Table 5.2. 
Due to the characteristics of the proposed protocol, every node has two channels, and a maximum of two neighbors, one per channel, during the transmission process. This means that the number of nodes contending the channel will be $n=2$.

Table 5.2: Parameter values used to validate the saturation throughput.

\begin{tabular}{|c|l|c|l|}
\hline Window size & $8,16,32$ packets & Data rate & $19600 \mathrm{~b} / \mathrm{s}$ \\
\hline Bit error rate & $1 \times 10^{-3}, 1 \times 10^{-4}$ & Distance & $50 \mathrm{~m}$ \\
\hline ACK packet size & $30 \mathrm{bits}$ & Overhead & $8 \mathrm{bits}$ \\
\hline Speed of sound & $1500 \mathrm{~m} / \mathrm{s}$ & Packet size & $150,300,600,1200,2400,4800 \mathrm{bits}$ \\
\hline
\end{tabular}



Figure 5.3: Throughput comparison with $B E R=1 \times 10^{-3}$.

Figures 5.3 and 5.4 show that simulation results are really close compared with the results given by the analytical model, meaning that the analytical model of the throughput is accurate, representing as a consequence the throughput of $2 \mathrm{MAC}$. There is a huge throughput improvement in Figure 5.4 since the BER is $1 \times 10^{-4}$, meaning that the probability of having errors in a packet arrived to the receiver is very low compared with the BER in Figure 5.3, and there will indeed be fewer retransmissions. 


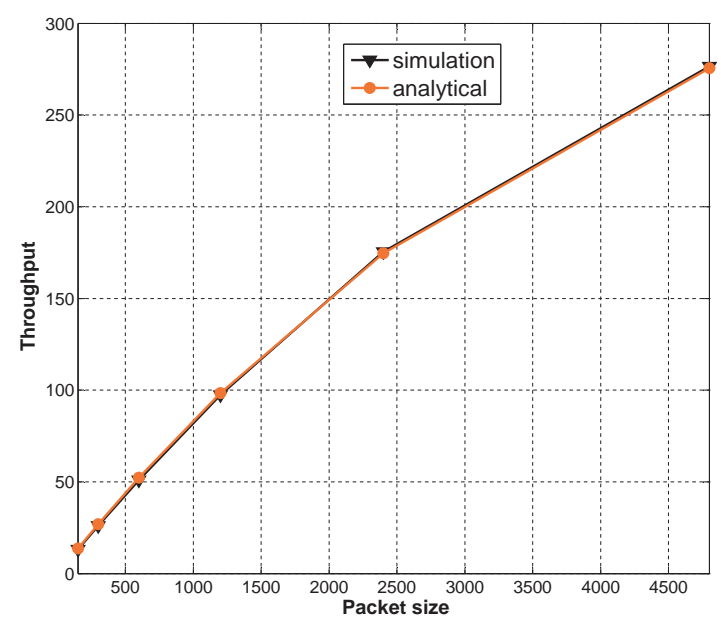

Figure 5.4: Throughput comparison with $B E R=1 \times 10^{-4}$.

\subsection{Analytical Model for SW-MER}

This section presents the analytical model to calculate the throughput of the SW-MER protocol. The throughput is obtained as a function of the packet size, bit rate and the expected bit error probability. The analysis assumes a finite number of nodes in the network. The remainder of this section is organized as follows. Section 5.2.1 includes a brief description of some components needed for the transmission process at the logical link level for underwater communications. Section 5.2.2 describes the throughput analysis, and Section 5.2.3 presents the analytical model validation.

\subsubsection{SW-MER Transmission Process}

The process implemented in SW-MER for sending packets is similar to the one designed in [33], however the difference is in the number of copies sent for every packet arrived with errors, in the next window retransmission. 
Table 5.3: Notations used to obtain the throughput efficiency.

\begin{tabular}{|c|l|l|l|}
\hline$T_{\text {prop }}$ & Packet propagation delay & $T_{t x d}$ & Data packet transmission time \\
\hline$T_{\text {procd }}$ & Data packet processing delay & $T_{t x a}$ & Acknowledgment packet transmission time \\
\hline$T_{\text {proca }}$ & Acknowledgment packet processing delay & $T_{\text {tot }}$ & Total time needed to send the data \\
\hline
\end{tabular}

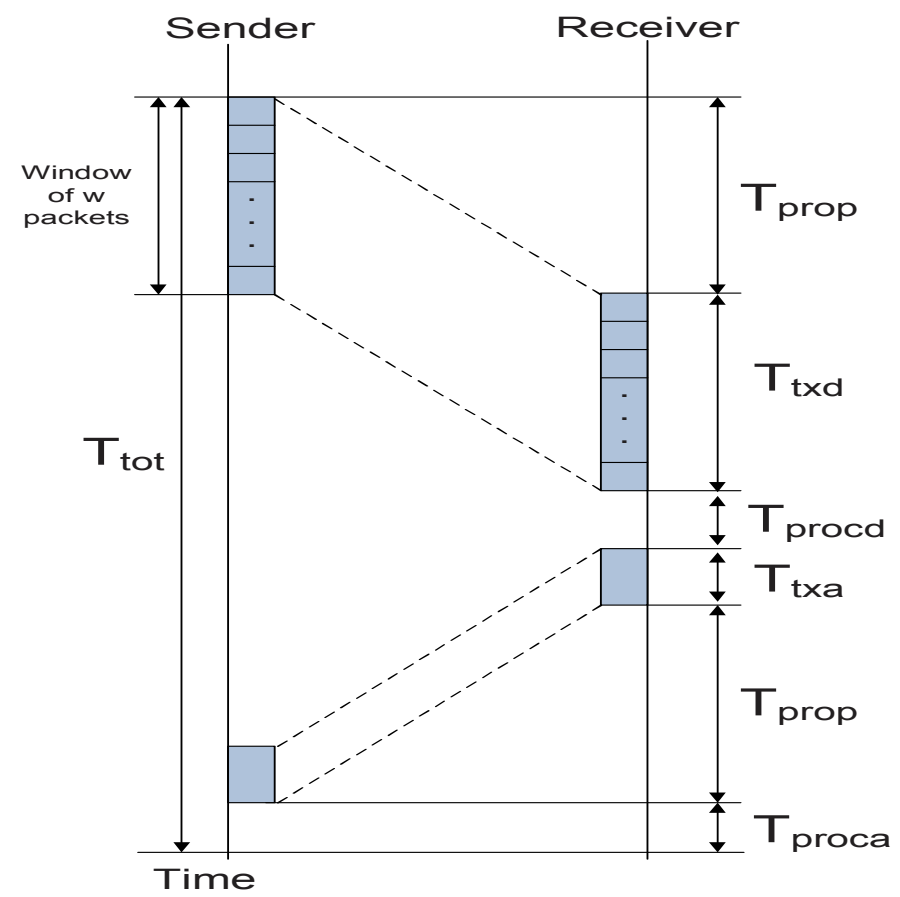

Figure 5.5: SW-MER, transmission process.

As explained in chapter 4, this is exponentially increased every time the packet is retransmitted. Figure 5.5 shows the times involved in the transmission of a data packet using the SW-MER protocol, and Table 5.3 describes each of the variables. Following the figure, the total time $T_{\text {tot }}$ needed for the transmitter to send a window of $w$ data frames and receive the corresponding acknowledgment packet, is calculated as follows:

$$
T_{t o t}=T_{t x d}+T_{t x a}+2 \cdot T_{\text {prop }}+T_{\text {procd }}+T_{\text {proca }}
$$


Factors like the switch time spent either in the transmitter or the receiver to go to the listening state to the sending state also can affect the total time. If $T_{\text {procd }}$ and $T_{\text {proca }}$ are assumed negligible and the switch time is included, Equation 5.51 can be rewritten as follows:

$$
T_{t o t}=T_{t x d}+T_{t x a}+2\left(T_{\text {prop }}+T_{s w}\right)
$$

In the next section the throughput is derived.

\subsubsection{Throughput Analysis}

The analytical model for the throughput is solved as in [35], computing the throughput efficiency of the SW-MER protocol. It is assumed that $D_{f}=D_{d}+D_{o h}$ in which $D_{f}$ is the size of each data frame in bits, $D_{d}$ is the length of the data to be transmitted in bits, and $D_{o h}$ is the packet overhead.

The size of the acknowledge frame in bits is represented as $D_{a c k}$, the speed of the sound underwater is by $c$ where $c=1500 \mathrm{~m} / \mathrm{s}, d$ is the distance between transmitter and receiver, and $r$ is the data rate in bits. Based on these variables, the propagation delay can be cal-

culated as $T_{\text {prop }}=\frac{d}{c}$, the transmission time needed to send a window of data frames as $T_{t x d}=w \cdot \frac{D_{f}}{r}$, the switch time as $T_{s w}=\frac{16}{r}$ (formula taken from [35]), and the transmission time needed to send an acknowledge packet as $T_{t x a}=\frac{D_{a c k}}{r}$. The equation for the throughput efficiency is represented in the following equation: 


$$
\eta=\frac{T_{d}}{T_{f}}
$$

$T_{d}$ represents the time necessary to transmit a window of $w$ data packets and $T_{f}$ is the average time needed to transmit a window of $w$ data frames successfully. $T_{d}$ is obtained in Equation 5.54, and $T_{f}$ in Equation 5.55.

$$
T_{d}=\frac{w \cdot D_{d}}{r}
$$

$$
T_{f}=E[w] T_{t o t}
$$

$E[w]$ is the average number of trials needed to transmit a window of $w$ packets successfully and is calculated as follows,

$$
\begin{array}{r}
E[w]=\sum_{k=0}^{\infty}\left(2^{k-1} \cdot P^{2^{k-1}-1} \cdot 2^{k-1} \cdot(1-P)\right)= \\
=\sum_{k=0}^{\infty}\left(4^{k-1} \cdot P^{2^{k-1}-1} \cdot(1-P)\right)
\end{array}
$$

$P$ is the packet error probability and is calculated as $P=1-(1-B E R)^{D_{f}}$, where $B E R$ represents the bit error probability or bit error rate in the channel, and $D_{f}$ represents the 
size of the data frame in bits. The probability of getting at least one packet without errors arriving to the receiver in the $2^{k-1}$ trial is calculated as $P^{\left(2^{k-1}-1\right)} \cdot 2^{k-1} \cdot(1-P)$.

Taking into account the equations presented before, the throughput efficiency in Equation 5.53 can be represented also in terms of the length of the data to be transmitted as follows:

$$
\eta=\frac{D_{d}}{[1-B E R]^{D_{d}+D_{o h}}\left[\sum_{k=0}^{\infty}\left(4^{k-1} \cdot\left(1-(1-B E R)^{D_{d}+D_{o h}}\right)^{\left(2^{(k-1)-1}\right)}\right)\right]\left[D_{d}+e\right]}
$$

in which $e$ is,

$$
e=\frac{D_{a c k}}{w}+\frac{2 \cdot r\left(T_{s w}+T_{\text {prop }}\right)}{w}+D_{o h}
$$

The process to obtain the Equation 5.58 is described in Appendix B.

\subsubsection{Model Validation}

To validate the analytical model, the results have been compared with the ones obtained with the simulations in chapter 4 . The values of the parameters used to obtain the results for both the simulation program that represents the proposed data link protocol and the analytical model can be seen in Table 5.4. 
Table 5.4: Parameter values used to validate the throughput efficiency.

\begin{tabular}{|c|l|c|l|}
\hline Window size & $8,16,32$ packets & Data rate & $2400 \mathrm{~b} / \mathrm{s}$ \\
\hline Bit error rate & $1 \times 10^{-3}$ & Distance & $50 \mathrm{~m}$ \\
\hline ACK packet size & $30 \mathrm{bits}$ & Overhead & $8 \mathrm{bits}$ \\
\hline Speed of sound & $1500 \mathrm{~m} / \mathrm{s}$ & Packet size & $150,300,600,1200,2400,4800 \mathrm{bits}$ \\
\hline
\end{tabular}

Figures 5.6, 5.7, and 5.8 show the comparison of the analytical and simulation results of the SW-MER protocol. As it can be seen, the results are very close, meaning that the analytical model of the throughput efficiency is accurate, representing the throughput of SW-MER.

The results shown in the figures are just for validation purposes, the chapter 6 presents the evaluation of the SW-MER protocol.

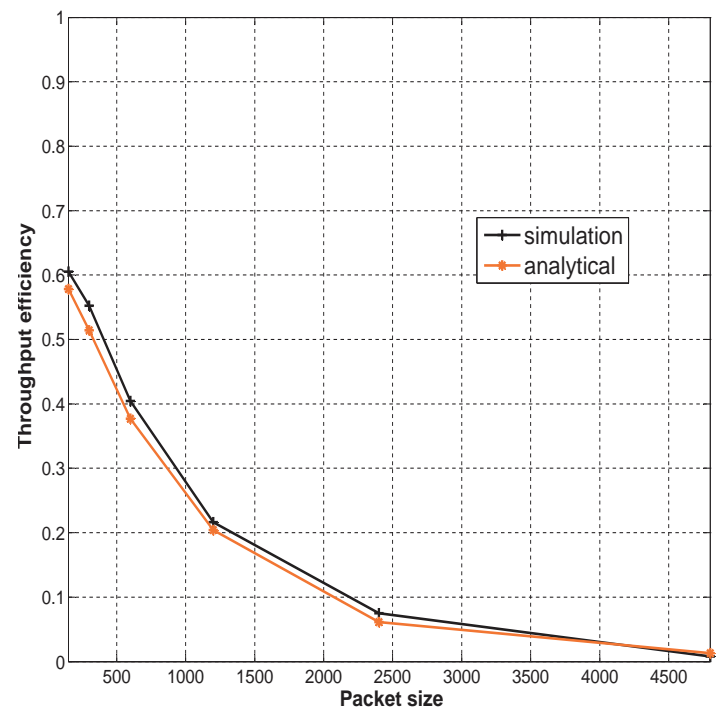

Figure 5.6: Throughput efficiency comparison with a window size of 8 packets. 


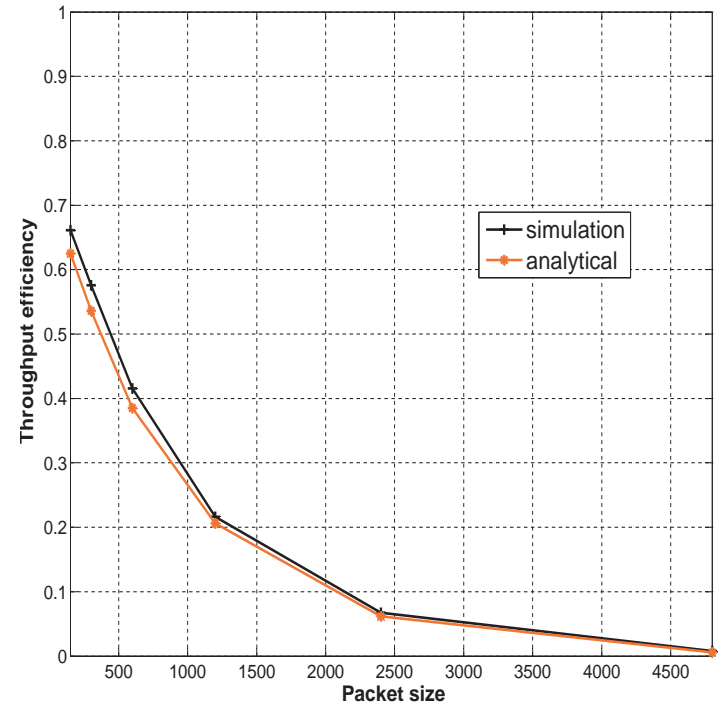

Figure 5.7: Throughput efficiency comparison with a window size of 16 packets.

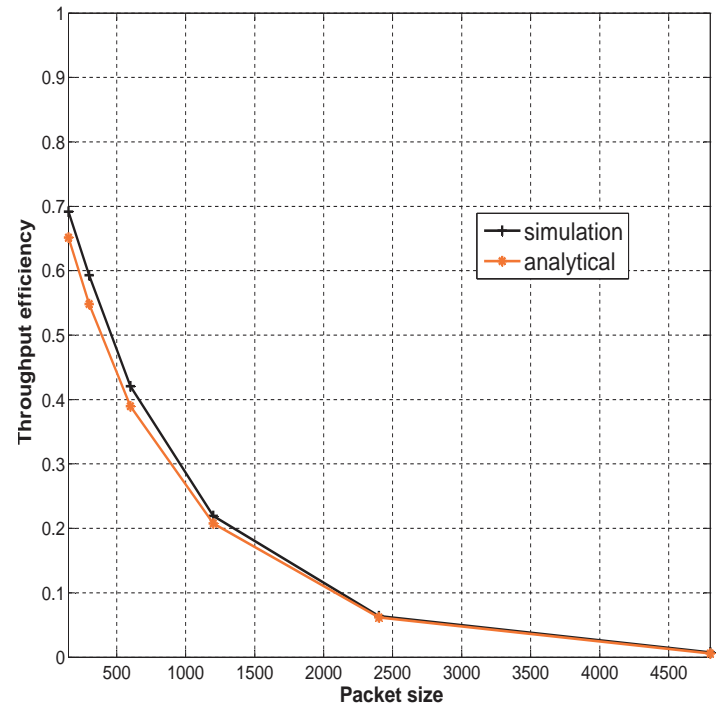

Figure 5.8: Throughput efficiency comparison with a window size of 32 packets. 


\section{Chapter 6: Performance Evaluation}

This chapter presents the results of the performance evaluation of the 2MAC and SWMER protocols. Three sets of results are included. The first and second sets include the evaluation of the MAC protocols and the logical link control protocols alone, respectively. The third set presents the evaluation of the SW-MER and 2MAC protocols together (the data link layer).

\subsection{Scenarios and Parameters}

In all experiments the maximum number of trials applied in the protocols [49], [33] and [36] is the maximum assigned by the 802.11 for data packets, 4 . To obtain the packet error probability, based on the BER, Equation 6.1 in which $D_{f}=D_{d}+D_{o h}$ was applied. The evaluation is performed using two error models. The first one corresponds to the Bernoulli model and the second one to the channel characterization results presented in [45]. The scenarios in which the protocols were evaluated are linear networks with four nodes. The parameters used in the evaluations can be seen in Table 5.4. These error models are explained in more detail next. 
Table 6.1: Parameter values used to evaluate 2MAC and SW-MER.

\begin{tabular}{|c|l|c|l|}
\hline Window sizes & 8,16 packets & Data rate & $2400 \mathrm{~b} / \mathrm{s}$ \\
\hline Bit error rate & $1 \times 10^{-3}, 1 \times 10^{-4}$ & Distance & $50 \mathrm{~m}$ \\
\hline ACK packet size & $30 \mathrm{bits}$ & Overhead & 8 bits \\
\hline Acoustic speed & $1500 \mathrm{~m} / \mathrm{s}$ & Packet sizes & a) $150,300,600,1200,2400,4800$ bits \\
\hline & & Packet sizes & b) $100,200,300,400,500,600$ bits \\
\hline sinchronization time & $0.16 \mathrm{~s}$ & & \\
\hline
\end{tabular}

\subsection{Channel Error Models}

In order to evaluate the performance of the data link control protocols under consideration, two channel error models were utilized. The first model corresponds to the simple Bernoulli model in which the Packet Error Probability (PER) can be easily calculated as:

$$
P E R=1-(1-B E R)^{D_{f}}
$$

where $N$ is the number of bits in the packet and BER is the Bit Error Rate.

The second model named as the Markov model error channel, was developed by modeling the errors of an underwater channel using a similar approach as the ones described in [50] and [51], where a two-state Markov chain is used to model the errors in wireless communications channels. The first step in the model generation is the characterization of the channel. It is important to take into account that real measurements were not performed; instead, the channel characterization results presented in [45] were used, where real measurements were taken using acoustic communications in shallow water (depth $15-20 \mathrm{~m})$ at a range of $50 \mathrm{~m}$.

From [45], the channel impulses (the amplitudes and the values) were used to recreate the channel behavior, including the effect of mobility considering the doppler effect while moving at $1 \mathrm{~m} / \mathrm{sec}$, a depth of $4 \mathrm{~m}$, and a distance of 50m. Using Matlab simulations, a 


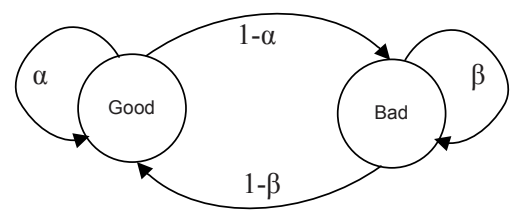

Figure 6.1: Two-state Markov model representation.

trace of 10 million impulses was generated using Orthogonal Frequency-Division Multiplexing (OFDM) and Binary Phase Shift Keying (BPSK) modulation schemes. The generated impulse results, $+1,-1$ and 0 were taken as a representation of bit with errors $(+1$ and -1 values $)$ and bits without errors ( 0 value). Then, with this trace, the transition probability matrix $A$ and the error probability matrix $B$ of the two-state Markov chain shown in Figure 6.1 were obtained. Given initial values of $A$ and $B$, a Hidden Markov Model (HMM) approach was taken to generate the channel error model [52]. In this process the well-known Baum-Welch algorithm [53] was used to find the unknown parameters of the HMM. The initial values of the $A$ and $B$ matrices $\left(A_{0}\right.$ and $\left.B_{0}\right)$ as well as the final values, final steady state matrices $A_{s s}$ and $B_{s s}$, found by the model are as follows:

$$
\begin{gathered}
A_{0}=\left[\begin{array}{ll}
0.98 & 0.02 \\
0.05 & 0.95
\end{array}\right] A_{s s}=\left[\begin{array}{ll}
0.8116 & 0.1884 \\
0.0095 & 0.9905
\end{array}\right] \\
B_{0}=\left[\begin{array}{ll}
0.9 & 0.9 \\
0.1 & 0.1
\end{array}\right] B_{s s}=\left[\begin{array}{ll}
0.9909 & 0.68 \\
0.0091 & 0.32
\end{array}\right]
\end{gathered}
$$

After doing some simulations using the Markov model error channel over 10,000,000 bits sent by the transmitter, the amount of bits with errors arrived to the receiver is obtained, and also the average BER generated. The BER obtained is $2.4 \times 10^{-2}$, meaning that this error channel is high in errors. 


\subsection{Performance Evaluation for 2MAC}

The experiments at the MAC layer compare the IEEE 802.11 protocol with the proposed 2MAC protocol using both a channel speed of $2400 \mathrm{bps}$ and packet sizes of 1200 and 2400 bits. The protocols were evaluated considering a linear network of four nodes 50m apart simulating a swarm of AUVs mapping the ocean floor without errors and without the logical link control sublayer.

The traffic in the network was generated as follows. Each node generated flowed to all other nodes, sending packets according to a Poisson process. The rate of the flows was set so that the load in the network was set to the specific desired level. In addition, a tagged flow sending packets from node one (left extreme of the network) to node four (right most node) was established and monitored. The performance results shown in all graphs are related to the performance of this tagged flow, which, as a reference, is indicated in the plot by a dotted line with a maximum possible throughput of $60 \mathrm{bps}$ or $30 \mathrm{bps}$ in the case of packet sizes of 2400 and 1200 bits, respectively. A simple network layer was included on top of the MAC layer to route incoming packets to the following adjacent node and the assumption of an underwater channel without errors. The objective was to analyze the amount of collisions produced for each MAC protocol under high traffic level in the network.

Figure 6.2 and 6.3 show the throughput results of the MAC protocols. As it can be seen from the figures, the traffic load of the network is increased from $10 \%$ to $100 \%$ and $2 \mathrm{MAC}$ still presents a better throughput performance compared with the traditional CSMA/CA protocol. This can be easily explained by the use of multiple channels, which reduces the number of collisions. Also, packets are sent through both channels in 2MAC increasing 




Figure 6.2: Throughput of the MAC protocols, packet size of 1200 bits.

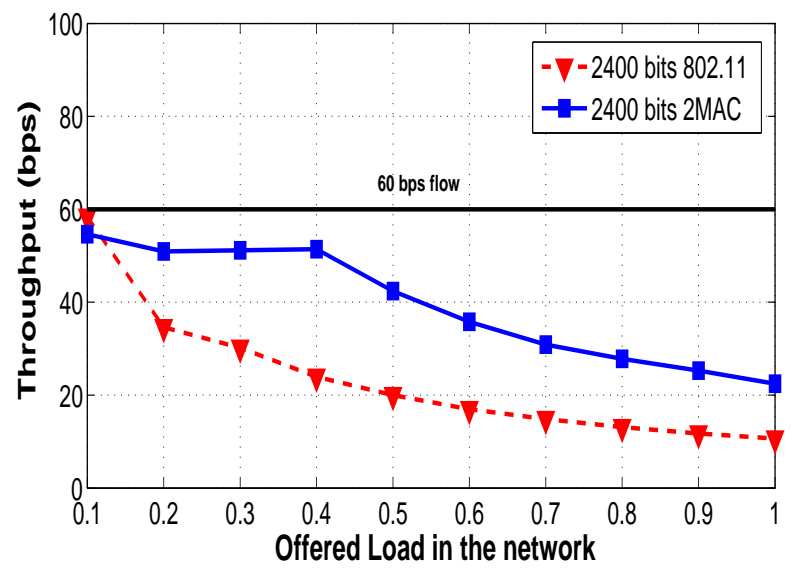

Figure 6.3: Throughput of the MAC protocols, packet size of 2400 bits.

the throughput, but in CSMA only one channel is used for the transmission.

\subsection{Performance Evaluation for SW-MER}

In this section, the performance of the proposed SW-MER protocol is compared with the stop and wait protocols described in [49], [33] and [36] (shown in the plots as sw2, $s w 3$, and $s w 4$, respectively). The stop and wait described in [49] works by sending one 
packet all the time from the sender. Once the packet arrives to the receiver, it verifies if the packet arrives with or without errors and sends an acknowledgment to the transmitter. Then the transmitter will retransmit the packet until it arrives without errors to the receiver.

The stop and wait variant proposed in [33] works by transmitting a window of $m$ packets all the time. The transmitter sends $m$ packets in every transmission opportunity and waits for the corresponding acknowledgments sent by the receiver in one packet that includes all the information. Once the acknowledgments are received, the transmitter verifies which packets arrived with errors, and sends a new window of $m$ packets filled with those received in error from the past window, and new packets if space in the new window is available. The process continues until all the packets are transmitted. The stop and wait version described in [36] is very similar to the one in [33], working with a window of packets. However, upon receiving the acknowledgment packet, the transmitter sends a new window containing only the packets received in error. Once these packets are correctly received, the sender sends a new window with $m$ new packets.

The evaluation of the protocols was performed using the two error models described in the last section, and the performance metrics utilized were throughput efficiency and packet delivery rate. This last metric indicates the level of reliability provided by each protocol. The experiments use a channel speed of 2400 bps and packet sizes of 300, 600, 1200, 2400, and 4800 bits for the Bernoulli model, and packet sizes of 100, 200, 300, 400, 500, and 600 bits for the Markov channel error model. The protocols were evaluated considering a point to point transmission between two nodes $50 \mathrm{~m}$ apart from each other and without the existence of the MAC control sublayer. Traffic was generated according to a Poisson process. 




Figure 6.4: Throughput efficiency for a window size of 8 data packets using the Bernoulli error model.



Figure 6.5: Throughput efficiency for a window size of 16 data packets using a Bernoulli error model. 




Figure 6.6: Packet delivery rate for a window size of 8 data packets using the Bernoulli error model.

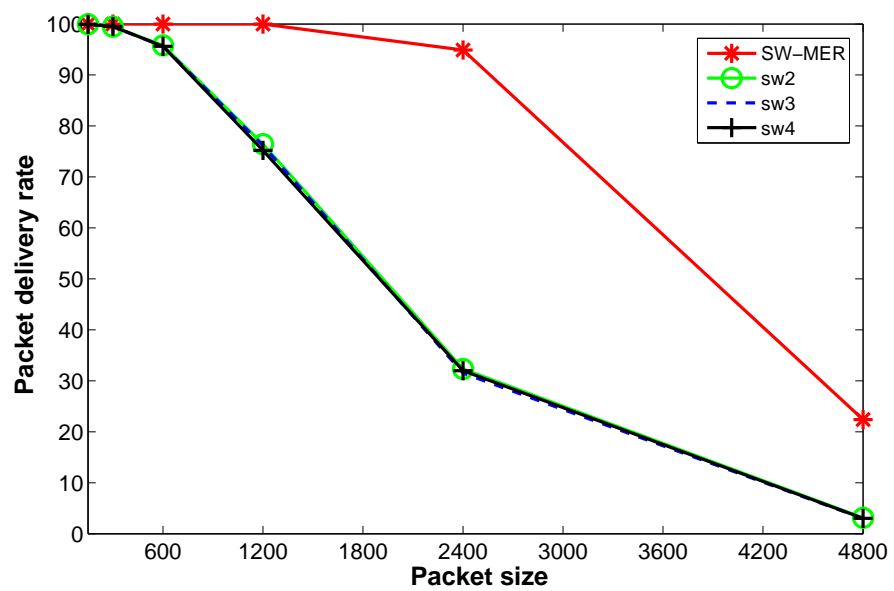

Figure 6.7: Packet delivery rate for a window size of 16 data packets using the Bernoulli error model. 


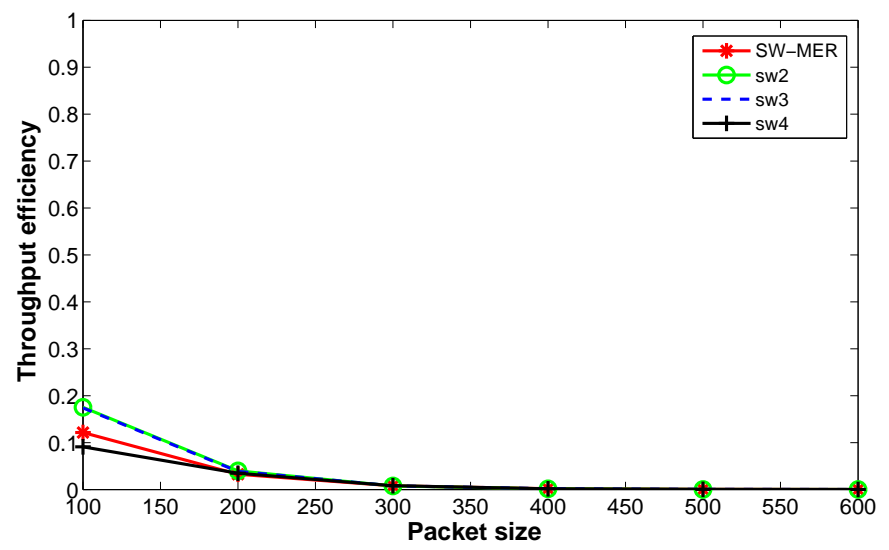

Figure 6.8: Throughput efficiency for a window size of 16 packets using the shallow water error model.

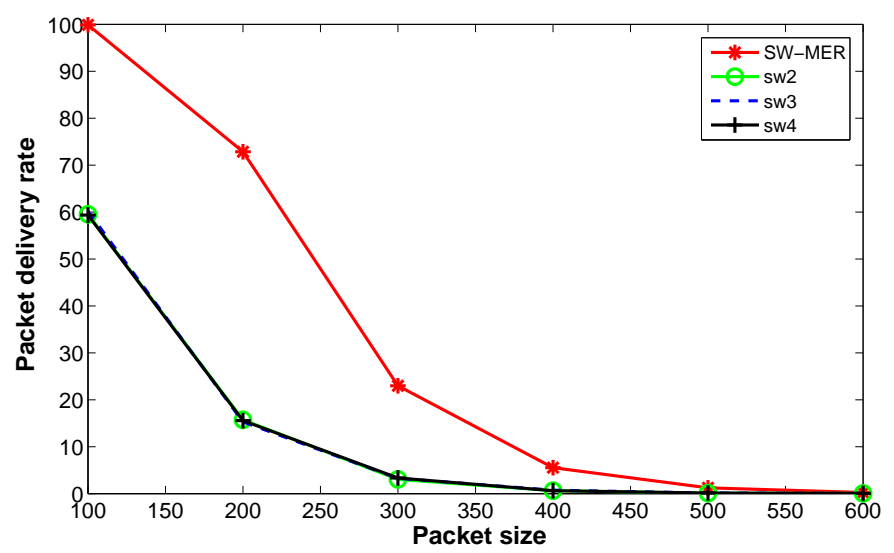

Figure 6.9: Packet delivery rate for a window size of 16 data packets using the shallow water error model. 
Figures 6.4 and 6.5 show the throughput efficiency of the logical link control protocols using a window size of 8 and 16 packets, respectively. As it can be seen, regardless of the BER, all protocols present a similar performance. The benefit of the SW-MER protocol is in the packet delivery ratio, as shown in Figures 6.6 and 6.7, based on a channel with a BER of $10^{-3}$. As expected, the performance of the protocols decreases with the packet size. However, the superiority in reliability of the SW-MER protocol is demonstrated. This is due to the exponential increase packet retransmission strategy utilized by SWMER. In the case of a BER of $10^{-4}$ (results not shown here), all protocols experienced similar performance, indicating that SW-MER is better only in those extreme scenarios with very poor quality channels.

In the case of the underwater channel error model, similar experiments were performed to compare the results with the Bernoulli error model, using the trace, Markov model, and parameters described in Section 6.2. As it can be seen from Figure 6.8, the throughput efficiency is very low compared with the Bernoulli model, indicating that the underwater error model introduces a larger amount of errors. This is magnified considering that the experiments utilized smaller packet sizes. The packet delivery ratio of the protocols is shown in Figure 6.9. From these last plots, it can be concluded that in order to have an acceptable throughput and packet delivery ratio in an underwater channel, packets cannot be longer than 250 bytes. Similar results were found using smaller window sizes.

\subsection{Performance Evaluation for the Entire Data Link}

The performance of the tagged flow was also assessed in the same linear network using the same parameters in the last section but now using the proposed SW-MER and 2MAC 


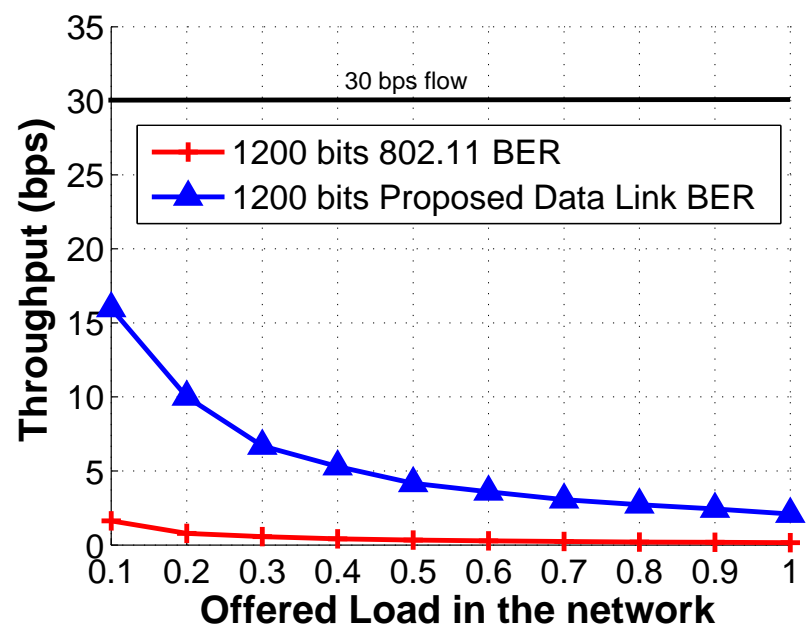

Figure 6.10: Throughput of the combined SW-MER and 2MAC protocols using packet size of 1200 bits, and $\mathrm{BER}=1 \times 10^{-3}$.

protocols together and including the Bernoulli and the underwater error models (shown in the plot as MM - Markovian Model). Figure 6.10 shows the performance of the tagged flow when sending packets of 1200 bits with a $B E R=1 \times 10^{-3}$. It is shown that having a packet of this size, the proposed data link protocol presents a better performance in terms of throughput than the traditional 802.11 protocol. Figure 6.11 presents the throughput performance but using the underwater Markov channel error model. Although both protocols are evaluated with a more realistic channel error model, the proposed data link protocol still has a better performance. The reason of having a better performance with both channel error models is because the proposed data link protocol uses two channels for simultaneous transmissions, and window stop and wait packet transmissions guaranteeing an increase in the throughput.

The same situation is presented in Figure 6.12 when packets of 2400 bits are sent. The proposed data link protocol presents again a better performance than the traditional 802.11 protocol, using an underwater error channel with a $\mathrm{BER}=1 \times 10^{-3}$. From both graphs, 


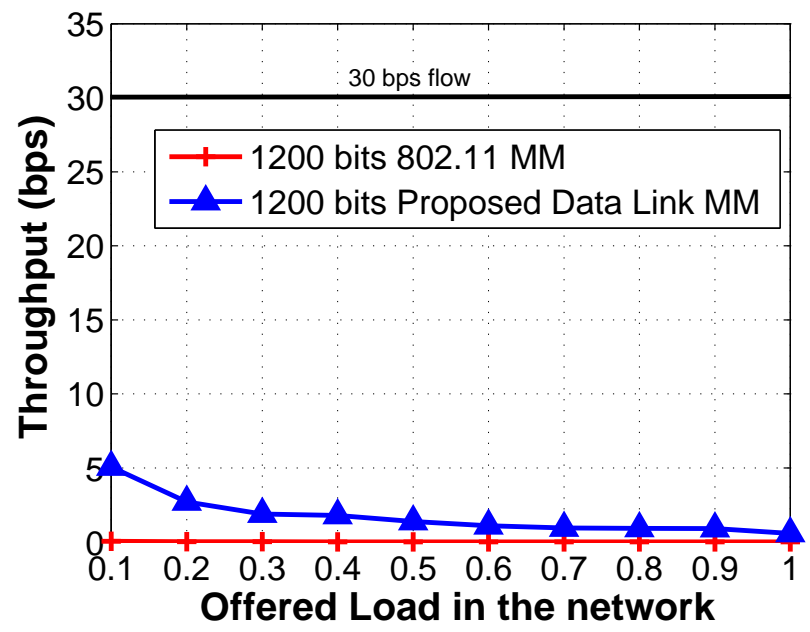

Figure 6.11: Throughput of the combined SW-MER and 2MAC protocols using packet size of 1200 bits, and Markov model channel.

in general three main conclusions can be drawn. First, the proposed MAC protocol, as expected, improves the performance over the CSMA/CA protocol. Second, if the packet size is increased, however the throughput presented by $2 \mathrm{MAC}$ is better, the performance of the network in the underwater channel decreases because the probability of having an error in each packet sent increases. Third, it is clear that the performance in all cases is fairly poor but even worse using the underwater channel Markov error model, indicating that more research is needed at all these layers to improve the performance of acousticbased underwater communication systems. 




Figure 6.12: Throughput of the combined SW-MER and 2MAC protocols using packet size of 2400 bits. 


\section{Chapter 7: An Adaptive Logical Link Sublayer Protocol in Response to Underwater Acoustic Communication (UAC) Channel Changes}

In order to improve the throughput and the reliability of the underwater communication, the SW-MER protocol was proposed in chapter 4, which includes a combination of stop and wait and window-based flow control strategy to improve the corresponding throughput, and an exponential retransmission strategy to improve the packet delivery ratio. However, the retransmission strategy of SW-MER uses a predefined number of packets for retransmission regardless of the channel condition. Although this strategy

improves the packet delivery ratio, as demonstrated in chapter 6 , there may be occasions where the protocol might be sending unnecessary copies of the same packet.

This chapter presents an adaptive logical link protocol that addresses this particular problem. The new protocol builds upon the logical link control protocol presented in chapter 4, improving the performance of the SW-MER protocol by making it adaptive to channel conditions without compromising the reliability of SW-MER. In the next sections, a description of the adaptive protocol is introduced, including how the quality of the channel is obtained, and some comparison evaluations with the SW-MER protocol as well. 


\subsection{Adaptive SW-MER Description}

The proposed protocol includes an adaptive retransmission strategy that changes the number of copies to be retransmitted according to the quality of the channel. A cross layer approach between the data link and the physical layer is assumed, in which the physical layer informs the state of the channel to the data link layer every time, in order to adapt the amount of copies of retransmitted packets in each window packet transmission as a reaction to channel changes, to improve the throughput of the network.

The process starts at the MAC layer when the RTS control packet is sent, and later the CTS including a value representing the state of the channel is received. Taking into account this state and the possible packets to be sent in the window transmission, the sender will fill the window of packets to be sent with those that should be sent and their corresponding number of copies to be retransmitted. Each number of copies can exponentially increase or decrease depending on the channel behavior history saved in the sender. The adaptive protocol will be explained in more detail in the next sections.

\subsection{Determining the Channel Quality}

Depending on the distance between transmitter and receiver, the channel conditions as seen by each one might be different. Further, if the nodes move, these conditions will change with time. Figure 7.1 shows the scenario where two underwater unmanned vehicles explore the ocean sea at different depths.

When the transmitting UAV sends information to the adjacent AUV, it does not know about the channel conditions present at the receiver. If the channel presents errors, those 




Figure 7.1: Transmission between nodes located at different depths.

will be reflected in the data packets when they arrive at the receiver. The receiver then creates a vector of acknowledgments indicating which packets were received with and without errors, and sends it to the transmitter.

Saving information about the last transmission is not enough to predict what the channel condition will be the next time. Increasing the number of copies exponentially all the time might not be necessary, and a better throughput could be achieved if that number changed according to the channel quality. Based on this information, the four-state machine shown in Figure 7.2 was designed to determine the number of copies to be sent per incorrect packet, which considers the status of the channel during the last two transmissions (history) plus the current packet. The entire process works as follows:



Figure 7.2: Four-state machine representation. 
- If the receiver received the last two packets correctly and the current one is also correct, the transmitter determines that the channel is in the Good-Good state. In this case, the sender will decrease the number of copies per retransmitted packet by half, i.e., $c=c \div 2$.

- If the receiver received the last two packets correctly and the current one is bad, the transmitter determines that the channel is in the Good-Bad state. In this case, the sender will increase the number of copies per retransmitted packet by two, i.e., $c=c \times 2$.

- If the last two packets were received good and bad, and the current one is good, the transmitter determines that the channel is in the Bad-Good state. In this case, the sender will decrease the number of copies per retransmitted packet by half, i.e., $c=c \div 2$

- If the last two packets were received bad and good, and the current one is bad, the transmitter determines that the channel is in the Good-Bad state. In this case, the sender will increase the number of copies per retransmitted packet by two, i.e., $c=$ $c \times 2$.

- If the last two packets were received bad and good, and the current one is good, the transmitter determines that the channel is in the Good-Good state. In this case, the sender will decrease the number of copies per retransmitted packet by half, i.e., $c=c \div 2$.

- If the last two packets were received good and bad, and the current one is bad, the transmitter determines that the channel is in the Bad-Bad state. In this case, the sender will increase the number of copies per retransmitted packet by two, i.e., $c=c \times 2$. 


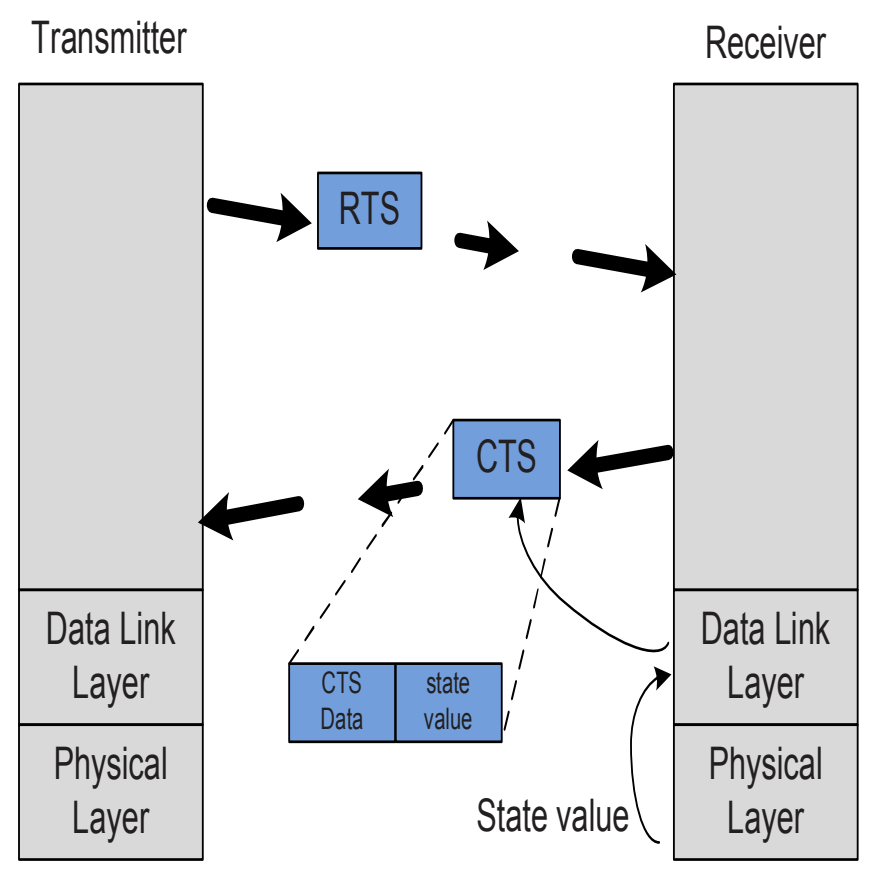

Figure 7.3: Physical and data link layer, interaction.

- If the receiver received the last two packets incorrectly and the current one is also incorrect, the transmitter determines that the channel is in the Bad-Bad state. In this case, the sender will increase the number of copies per retransmitted packet by two, i.e., $c=c \times 2$.

- If the receiver received the last two packets incorrectly and the current one is good, the transmitter determines that the channel is in the Bad-Good state. In this case, the sender will decrease the number of copies per retransmitted packet by half, i.e., $c=c \div 2$

Figure 7.3 illustrates the communication process between sender and receiver that conveys the channel quality information. 




Figure 7.4: Finite state machine of the sender process.

\subsection{States of the Adaptive Logical Link Protocol}

The adaptive SW-MER has the following states: Listen, Verifying the State of the Channel, Updating Channel Status History, Updating Copies of Packets to be Sent, Waiting to Receive Packets, Checking Packets, Enqueueing Packets without Errors, Verifying Packets to be Sent to the Upper Layer, Generating the ACK Vector, and Identifying Packets Sent with Errors. Figures 7.4 and 7.5 show the sender and receiver state machines for the new protocol, respectively. The states and state transitions needed in the sender and receiver processes are explained next. 


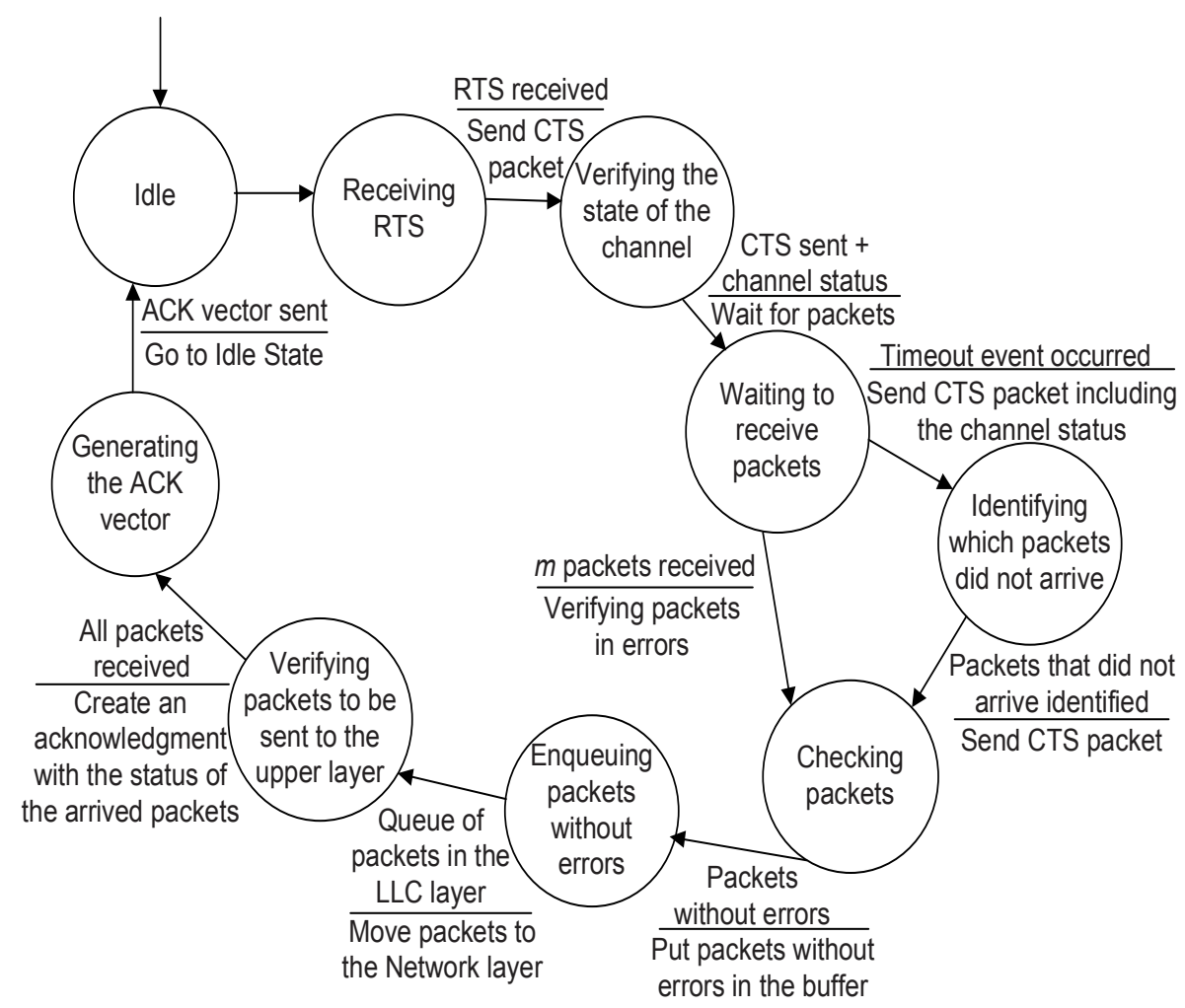

Figure 7.5: Finite state machine of the receiver process.

\subsubsection{Listen}

When a node decides to send packets to another node, it first selects the packets to be transmitted from the queue. Then an RTS is sent with the total time needed to transmit a window of $m$ packets and receive the acknowledgment vector.

\subsubsection{Verifying the State of the Channel}

Once the RTS arrives, the receiver saves the time that the transmission of $m$ packets will take and prepares the CTS packet. At this time the physical layer already has measured 
and identified the quality of the channel, and passed this information on to the data link layer. This information is added in the CTS packet, which is finally sent to the transmitter.

\subsubsection{Updating Channel Status History}

Once the CTS packet is received, the transmitter extracts the channel quality information and uses the four-state machine presented in Figure 7.2 to determine the number of times packets in error need to be retransmitted in the next transmission opportunity.

\subsubsection{Updating Copies of Packets to be Sent}

Based on the channel quality information and the four-state machine, the sender then assembles and transmits the new window of packets.

\subsubsection{Waiting to Receive Packets}

In this state, the receiver waits until the window of $m$ packets arrives or a timeout occurs because at least one of the packets never arrived. The receiver identifies the missing packets using two information fields in the header of the packets, one that represents the position of the packet in the window and another one that represents the ordinal copy number. 


\subsubsection{Checking Packets}

The receiver verifies if the packets arrived with or without errors by applying a wellknown Cyclic Redundancy Check (CRC) function, and classifies them accordingly.

\subsubsection{Enqueueing Packets Without Errors}

Packets that arrived without errors, are temporally enqueued in the buffer of the logical link layer. They stay there until a consecutive number of $m$ packets arrive without errors. Those packets that were received incorrectly are eliminated. In the proposed protocol, it is very easy to calculate the buffer size $B$ at the receiver to avoid packet drops, which is given by Equation 7.1 in which $w$ is the size of the window in number of packets and $t$ is the maximum number of trials that a data packet can be retransmitted. $t$ is obtained from Equation 7.2.

$$
\begin{gathered}
B=w \cdot t \\
t=\log _{2}(w)+1
\end{gathered}
$$




\subsubsection{Verifying Packets to be Sent to the Upper Layer}

Once a consecutive number of $m$ packets are received without errors, they are sent to the upper layer and deleted from the buffer. The purpose of sending the packets to the upper layer is to verify whether those packets belong to that node or must be retransmitted to the next neighbor.

\subsubsection{Generating the ACK Vector}

In this state, the receiver is in charge of creating an ACK control packet to tell the transmitter the state of each packet received. This ACK packet is a vector in which each position is a one bit value that represents the status of the packet, where 0 means that the packet arrived correctly, and 1 means that the packets arrived with errors or did not arrive at all. At the end, the ACK vector is sent to the transmitter.

\subsubsection{Identifying Packets Sent With Errors}

Once the ACK arrives to the sender, the vector is revised to identify which packets arrived with and without errors. Packets that arrived without errors are deleted from the sender's queue, the others are kept until a maximum number of trials is achieved or they are received correctly. The maximum number of trials is already defined in Equation 7.2. 


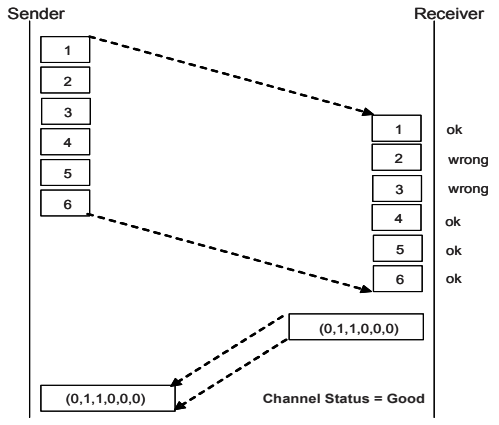

(a) First transmission, $M=6$ and packets 2 , and 3 arrive with errors.



(c) Third transmission, $M=6$ and packet 9 arrives with errors.

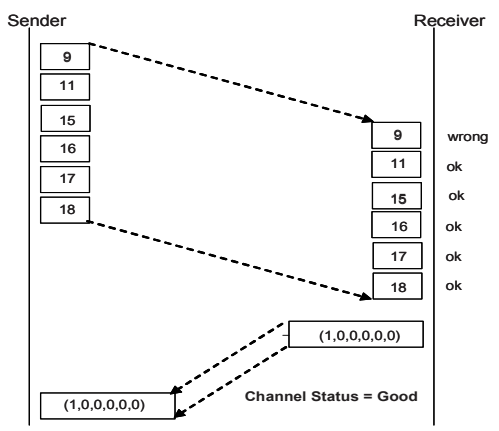

(e) Fifth transmission, $M=6$ and packet 9 arrives with errors.

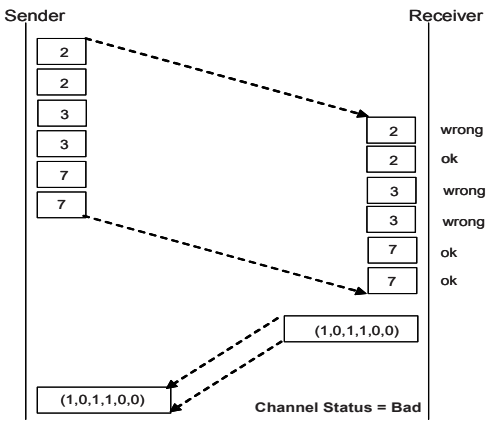

(b) Second transmission, $M=6$ and packet 3 arrives with errors again.



(d) Fourth transmission, $M=6$ and packet 9 and 12 arrive with errors.



(f) Sixth transmission, $M=6$ and packet 9 arrives without errors.

Figure 7.6: Example of the proposed adaptive stop and wait sliding window-based mechanism. 


\subsection{SW-MER Protocol Example}

Figure 7.6 shows an example of how the proposed protocol works using a window size of 6 packets. To define a transmission process as an RTS/CTS/DATA/ACK transmission, it is assumed that the channel was in a Good - Good state the last time, and a transmission process starts. Figure 7.6(a) displays the first transmission in which the channel status received in the CTS packet from the receiver indicates Good. Then, the state machine stays in Good - Good state, one copy of packets 1,2,3,4,5,6 are sent and 2,3 arrive with errors. The receiver sends an acknowledgment relaying this to the sender.

In the next transmission process, Figure 7.6(b), the transmitter sends two copies of the packets 2,3 because it received a Bad channel status in the CTS packet from the receiver. Also, the status of the state machine changes to a Good - Bad state. As displayed in Figure 7.6(c), the channel status still is Bad at the receiver. As a consequence, the state machine status goes to a $\mathrm{Bad}-\mathrm{Bad}$ state and the amount of copies of each packet to be sent will exponentially increase by 2 . Four copies of packet 3 are sent due to having arrived with error the last time. On the other hand, only two copies of packet 8 are sent because it is a new packet.

Figure 7.6(d) shows the fourth transmission. The transmitter is informed that the channel status is Good, then the state machine state status is updated to Bad - Good and the amount of copies of each packet to be sent is exponentially decreased by 2 . Since the transmitter is going to send new packets, only one copy from each one is sent. In Figure 7.6(e), only one copy of packets 9,11 are sent, although they arrived with errors in the previous transmission. This is because the last time the status of the channel state was Good-Good and still is Good. 


\subsection{Channel Error Models}

To evaluate the performance of both protocols, the same two channel error models used in Section 6.2 are considered.

\subsection{Performance Evaluation}

The performance evaluation of the proposed adaptive protocol is presented in this section. First, a general description of the parameters used in the simulations is presented. Then, the SW-MER and the adaptive SW-MER protocol are compared using the number of packet retransmissions and throughput as the main performance metrics.

\subsubsection{Simulation Parameters}

The simulation is carried out using the parameters included in Table 7.1. Nodes with linear formations are designed to evaluate the protocol. Two error models are considered, the Bernoulli model, and a synthetic trace obtained from [45]. The values of distance between nodes, data rate, and other related parameters are presented in the table. For the Bernoulli model a bit error rate of $1 \times 10^{-3}$ is used, control packet length's of 30 bits for RTS, CTS and ACK are applied in all the scenarios.

It is assumed a linear topology with 5 AUVs, as shown in Figure 7.7. The traffic in the network was generated as follows. Each node generated flows to all other nodes sending packets according to a Poisson process, with $\lambda$ of 4 data packets per second (expected arrival in a time interval). The rate of the flows is determined so that the load in the net- 
Table 7.1: Simulation parameters.

\begin{tabular}{c|c|c} 
Error Model & Parameters & Values \\
\hline \hline Bernoulli & Speed of sound & $1500 \mathrm{~m} / \mathrm{sec}$ \\
& Data rate & 2400 and $19600 \mathrm{~b} / \mathrm{s}$ \\
& Range & $50 \mathrm{~m}$ \\
& Packets per window & 8 \\
& Packet size & 150 and $300 \mathrm{bytes}$ \\
\hline$[45]$ & Speed of sound & $1550 \mathrm{~m} / \mathrm{sec}$ \\
& Data rate & $2400 \mathrm{~b} / \mathrm{s}$ \\
& Distance & $50 \mathrm{~m}$ \\
& Packets per window & 8 \\
& Packet size & 150 and 300 bytes
\end{tabular}

work is set to a specific desired level starting from 0 to $100 \%$. In addition, a tagged flow sending packets from node $\mathrm{E}$ to node $\mathrm{D}$ is established and monitored. The simulations results of the performance in all graphs are related to the performance of that tagged flow. A simple network layer is included on top of the LLC layer to route incoming packets to the following adjacent node.

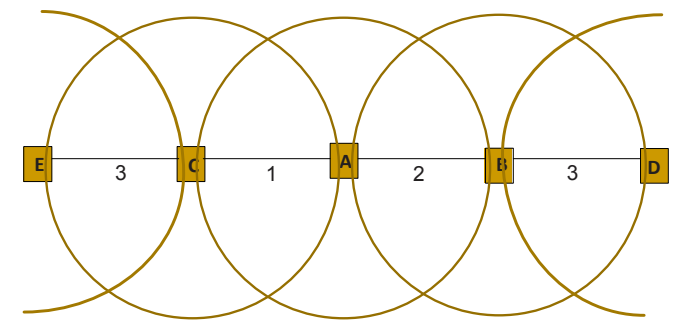

Figure 7.7: Linear topology.

\subsubsection{Throughput Evaluation}

In this section the performance of both protocols is compared using throughput and number of copies per packet sent as the main performance metrics. This second metric is 
defined as the average number of copies that need to be transmitted per packet so it is finally received correctly.

Figures 7.8, 7.9 and 7.10 show the amount of copies per packet generated by the two protocols. The first two scenarios were executed with the Bernoulli model using a BER of $1 \times 10^{-3}$ that generates a channel high in errors. As it can be seen, holding the same packet size and increasing the load in the network cause many packets to arrive with errors. Then the throughput of each protocol decreases, as seen in Figures 7.11 and 7.12. The SWMER protocol [9] reacts exponentially, increasing the number of copies per packet each time a transmission process starts, to guarantee a successful packet delivery. The protocol does not take into account that the channel is not in failure all the time like the proposed adaptive SW-MER. As expected however, the channel presents a lot of errors, and the proposed protocol adapts to channel changes generating fewer amount of copies to be sent, and improving the corresponding throughput, as seen in Figures 7.11 and 7.12.

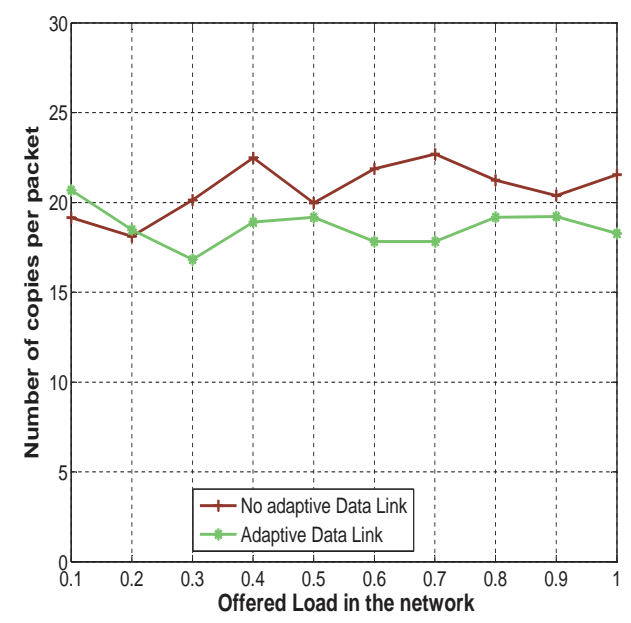

Figure 7.8: Copies per packet using a Bernoulli error model, a window size of 8 packets, a packet size of 2400 bits, $B E R=1 \times 10^{-3}$, and data rate of $2400 \mathrm{bps}$.

In the case of the underwater channel error model, similar experiments were performed to compare the results using the trace, Markov model, and parameters described in Sec- 




Figure 7.9: Copies per packet using a Bernoulli error model, a window size of 8 packets, a packet size of $2400 \mathrm{bits}, \mathrm{BER}=1 \times 10^{-3}$, and data rate of $19600 \mathrm{bps}$.

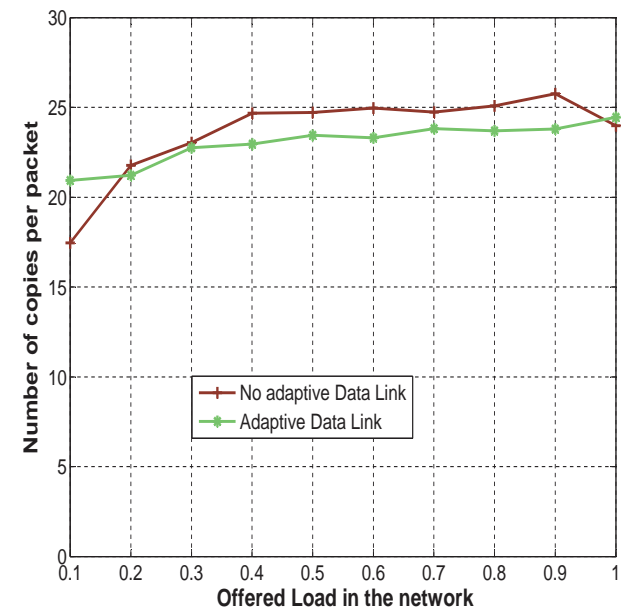

Figure 7.10: Copies per packet using a shallow water error model, a window size of 8 packets, a packet size of 1200 bits, and data rate of 2400 bps. 
tion 6.2. As it can be seen from the third scenario displayed in Figures 7.10 and 7.15, the number of copies generated per packet with the protocol in [9] starts to be bigger than the amount the proposed adaptive SW-MER produces as soon as the load of the network is increased, affecting the throughput. As it is shown in Figure 7.15, the throughput starts to deteriorate but still the superiority of the proposed adaptive SW-MER is demonstrated. This is as a consequence of the reduction of the number of copies retransmitted in each window, incrementing the number of new packets to be sent.

From the results plotted in Figures 7.8 and 7.9 for example, it can be seen that the adaptive protocol reduces the number of copies per packet that are generated compared with the amount generated by SW-MER, especially when the network is highly loaded. In addition, a throughput improvement can be obtained even using the synthetic trace that introduces a larger amount of errors in the order of 15\%, as shown in Figures 7.11, 7.12 and 7.16. Figures 7.13 and 7.14 show that for a window of 16 packets, however the throughput of both protocols are similar, the amount of packets generated for the previous protocol dramatically increases compared with the amount produced by the proposed adaptive SW-MER. 


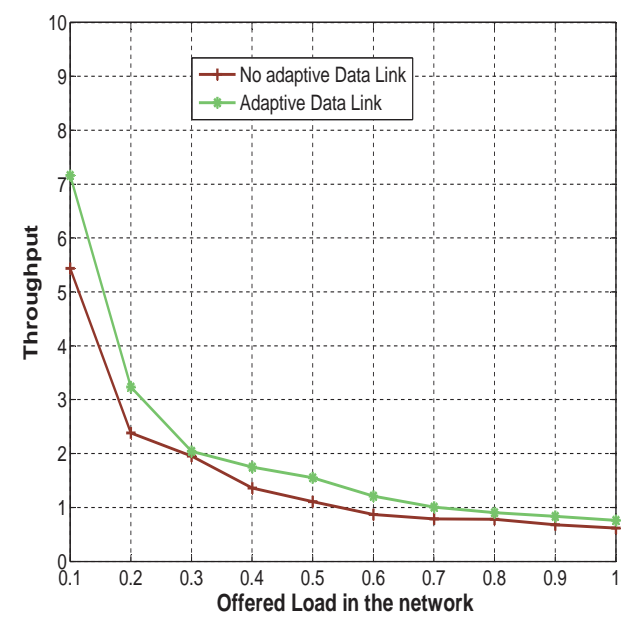

Figure 7.11: Throughput using a Bernoulli error model, a window size of 8 data packets, a packet size of 2400 bits, $B E R=1 \times 10^{-3}$, and data rate of $2400 \mathrm{bps}$.

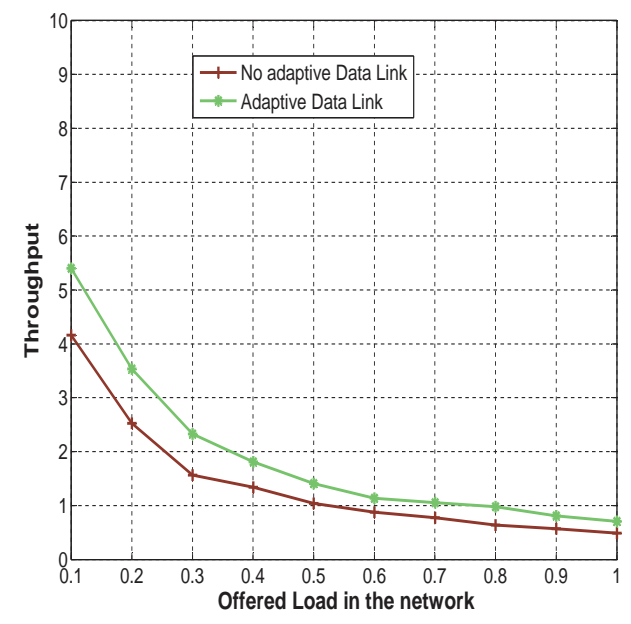

Figure 7.12: Throughput using a Bernoulli error model, a window size of 8 data packets, a packet size of 2400 bits, BER $=1 \times 10^{-3}$, and data rate of $19600 \mathrm{bps}$. 


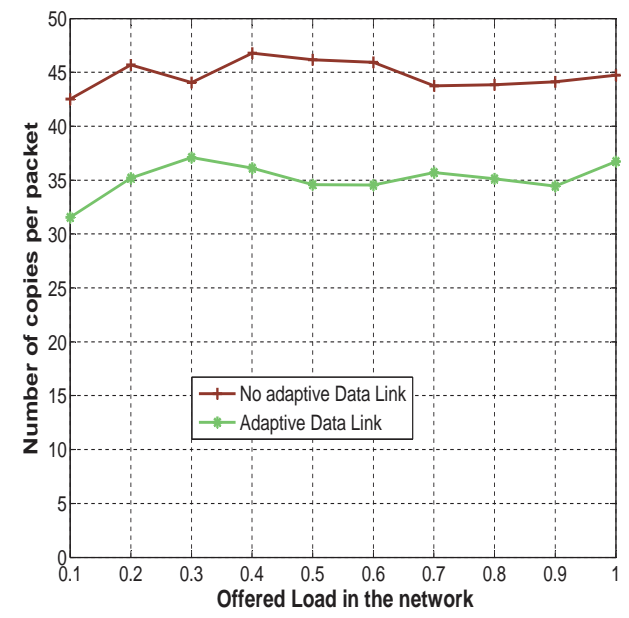

Figure 7.13: Copies per packet using a shallow water error model, a window size of 16 packets, a packet size of 2400 bits, and data rate of $19600 \mathrm{bps}$.

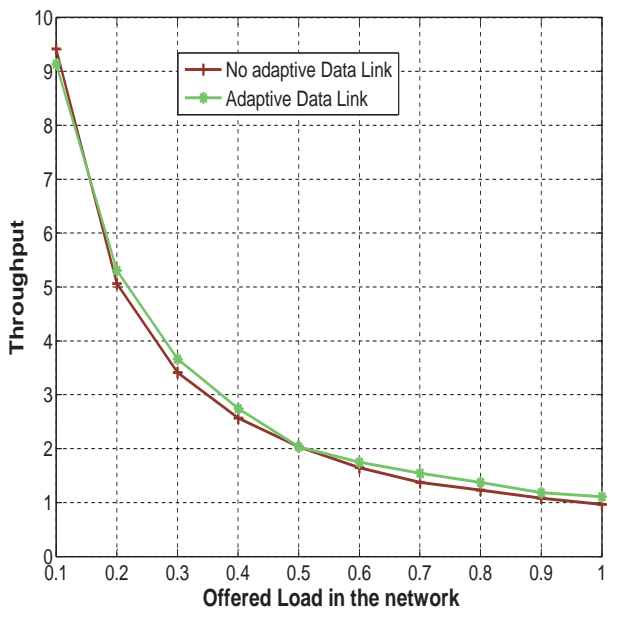

Figure 7.14: Throughput using a Bernoulli error model, a window size of 16 data packets, a packet size of 2400 bits, $B E R=1 \times 10^{-3}$, and data rate of $19600 \mathrm{bps}$. 


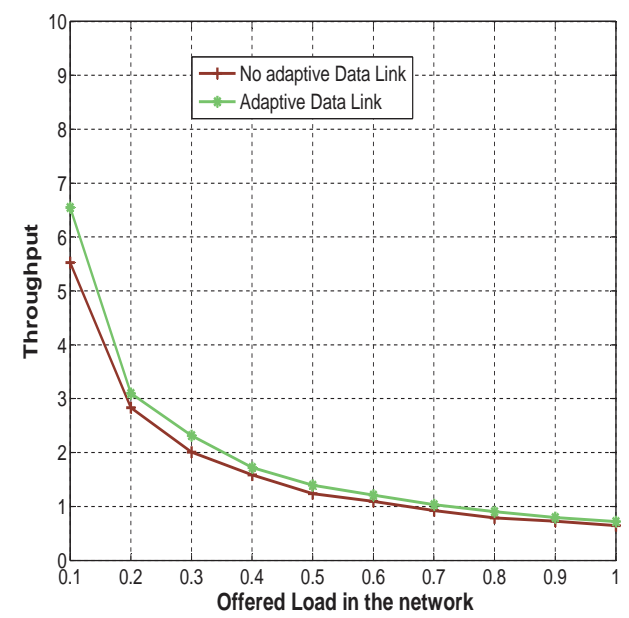

Figure 7.15: Throughput using a shallow water error model, a window size of 8 packets, and a packet size of 1200 bits.

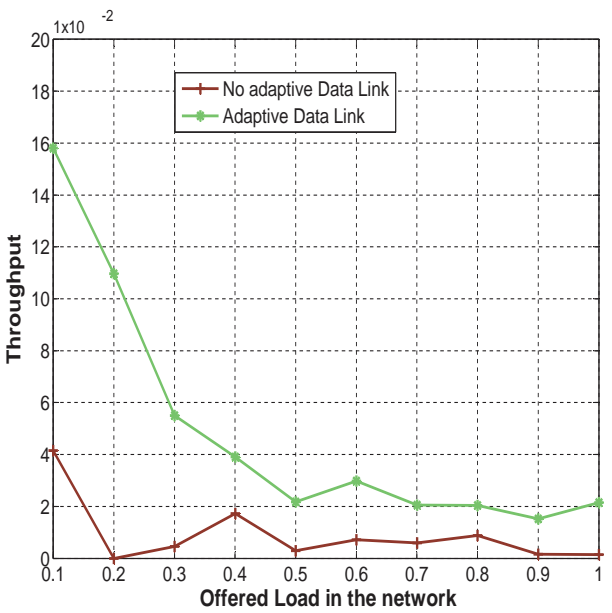

Figure 7.16: Throughput using a shallow water error model, a window size of 8 packets, and a packet size of 2400 bits. 


\section{Chapter 8: Conclusions and Future Work}

This dissertation proposes a new data link protocol designed for acoustic-based underwater communication systems, using OFDMA technology at the physical layer to support applications of swarms of autonomous underwater vehicles. This chapter presents the conclusions drawn from the contributions shown in Section 1.5 and outlines future directions of this work.

\subsection{Conclusions}

- A new MAC layer protocol, called 2MAC, designed to coordinate and take advantage of the multiple sub-channels made available by the use of OFDMA, is included. 2MAC uses three channels to transmit or receive data simultaneously from different neighbors without collisions and using only one transceiver. The proposed MAC protocol reduces collisions, and eliminates hidden terminal, exposed terminal and capture problems, improving as a consequence the throughput of the network. The 2MAC protocol shows its superiority over the well-known 802.11 protocol.

- A logical link control protocol, called SW-MER, is introduced. SW-MER combines stop and wait and sliding window mechanisms to increase the channel utilization and implements an exponential retransmission strategy to increase the packet deliv- 
ery ratio, especially under poor channel conditions like the ones commonly found underwater. By using the window packet transmission, the proposed LLC protocol also improves the throughput efficiency of the network.

- The combined SW-MER and 2MAC protocols are also evaluated as a complete data link protocol. The proposed data link protocol offers a better performance in terms of throughput than the 802.11 protocol in both channel error models used for the performance evaluation.

- A new backoff algorithm has been proposed for the 2MAC protocol. The throughput offered by the proposed backoff algorithm offers a similar throughput than the traditional backoff algorithms, however the size of the contention window in the new backoff is smaller. This is an advantage because the nodes do not have to spend a lot of time contending the channel before starting to transmit, especially in underwater communications where long propagation delays are present.

- An analytical model that represents the saturation throughput for $2 \mathrm{MAC}$ is shown.

- An analytical model that represents the throughput for the SW-MER protocol is also introduced.

- An improvement of the logical link protocol named adaptive SW-MER that works as a cross layer with the physical layer is shown. It is a logical link protocol that adapts to underwater acoustic communication channel changes for underwater vehicles. The superiority in terms of throughput of the adaptive protocol when compared with SW-MER is demonstrated. 


\subsection{Future Work}

The research developed in this dissertation can be extended for future work as follows:

- The underwater Markov channel error model designed represents an underwater channel for shallow water. An underwater channel error model for deep water should be designed to cover other underwater scenarios.

- The proposed data link layer protocol was evaluated over linear topologies. Evaluation of the proposed data link protocol over additional network scenarios such as those including polygonal formations, and using other underwater channel error models must be done.

- The analytical model proposed for the saturation throughput only includes the MAC sublayer. A more precise analytical model requires the inclusion of the LLC sublayer, to represent the saturation throughput for the proposed data link protocol.

- The implementation and evaluation of the proposed protocols have been applied in simulations. Implementing and evaluating these protocols over a real underwater network should give more precise results. 


\section{List of References}

[1] I. F. Akyildiz, D. Pompili, and T. Melodia, "State-of-the-Art in Protocol Research for Underwater Acoustic Sensor Networks," in Proceedings of the 1st ACM international workshop on underwater networks, 2006, pp. 7-16.

[2] M. Chitre, S. Shahabodeen, and M. Stojanovic, "Underwater acoustic communications and networking: Recent advances and future challenges," Marine Technology Society Journal, vol. 42, No.1, pp. 103-116, 2008.

[3] K. B. K. II and P. Mohapatra, "A Hybrid Medium Access Control Protocol for Underwater Wireless Networks," in Proceedings of the second workshop on underwater networks, September 2007, pp. 33-40.

[4] F. Schill, U. Zimmer, and J. Trumpf, "Visible Spectrum Optical Communication and Distance Sensing for Underwater Applications," in Proceedings of the Australian conference robotics and Automation (Canberra, 2004), 2004.

[5] B-C. Kim and I-T. Lu, "Parameter study of OFDM underwater communications system," in Proceedings of the IEEE Oceans conference and Exhibition, September 2000, pp. 1251-1255.

[6] S. Haykin, Communication Systems, 4th ed. John Wiley and Sons, 2001.

[7] A. Chandra and V. Gummalla and J. O. Limb, "Wireless Medium Access Control Protocols," IEEE Communications Surveys and Tutorials, 2000.

[8] J. W. Conard, "Services and Protocols of the Data Link Layer," Proceedings of the IEEE, vol. 71, No. 12, pp. 1378-1383, 1983. 
[9] D. Jabba and M. Labrador, "A Data Link Layer in Support of Swarming of Autonomous Underwater Vehicles," in Proceedings of the MTS/IEEE Oceans conference, Bremen, Germany, May 2009.

[10] — - "An Adaptive Logical Link Protocol in Response to Underwater Acoustic Communication Channel Changes for Autonomous Underwater Vehicles," in Proceedings of the MTS/IEEE Oceans conference, Biloxi, Mississippi, October 2009.

[11] M. Stojanovic, "Recent advances in high-speed underwater acoustic communications," IEEE Journal of Oceanic Engineering, vol. 21, No. 2, pp. 125-136, 1996.

[12] — - "Low Complexity OFDM Detector for Underwater Acoustic Channels," in Proceedings of the IEEE Oceans conference, September 2006.

[13] L. Freitag, M. Stojanovic, D. Kilfoyle, and J. Preisig, "High Rate Phase-Coherent Acoustic Communication: A Review of a Decade of Research and a Perspective on Future Challenges," in 7th European Conference on Underwater Acoustics, July 2004.

[14] J. Huang, J. Sun, C. He, X. Shen, and Q. Zhang, "High-speed underwater acoustic communication based on OFDM," in Microwave, Antenna, Propagation and EMC Technologies for Wireless Communications, 2005. MAPE 2005. IEEE International Symposium, August 2005, pp. 1135-1138.

[15] R. F. Ormondroyd, "A robust underwater acoustic communication system using OFDM-MIMO," in Proceedings of the MTS/IEEE Oceans conference, June 2007.

[16] S. Roy, T. M. Duman, V. McDonald, and J. G. Proakis, "High rate communication for underwater acoustic channels using multiple transmitters and space-time coding: Receiver structures and experimental results," IEEE Journal of Oceanic Engineering, February 2007.

[17] G. G. Xie and J. Gibson, "A networking protocol for underwater acoustic networks," Naval Postgraduate School, Tech. Rep. TR-CS-00-02, December 2000. 
[18] J.-K. Yeo, Y.-K. Lim, and H.-H. Lee, "Modified mac(media access control) protocol design for the acoustic-based underwater digital data communication," IEEE, pp. 364-368, 2001.

[19] E. M. Sozer, M. Stojanovic, and J. G. Proakis, "Underwater acoustic networks," IEEE journal of Oceanic Engineering, vol. 25, No. 1, pp. 72-83, 2000.

[20] H. Doukkali and L. Nuaymi, "Analysis of MAC protocols for underwater acoustic data networks," Vehicular Technology Conference, 2005. VTC 2005-Spring 2005 IEEE 61st, vol. 2, pp. 1307-1311, 2005.

[21] M. Molins and M. Stojanovic, "Slotted FAMA: A MAC Protocol for Underwater Acoustic Networks," in Proceedings of the IEEE Oceans conference, 2006.

[22] M. K. Park and V. Rodoplu, "UWAN-MAC: An Energy-Efficient MAC Protocol for Underwater Acoustic Wireless Sensor Networks," IEEE Journal of Oceanic Engineering, vol. 32, No. 3, pp. 710-720, July 2007.

[23] A. A. Syed and J. Heidemann, "Time synchronization for high latency acoustic networks," in Proceedings of the IEEE INFOCOM conference, April 2006.

[24] A. A. Syed, W. Ye, and J. Heidemann, "T-Lohi: A New Class of MAC Protocols for Underwater Acoustic Sensor Networks," in Technical Report ISI-TR-638, USC/Information Sciences Institute, 2007. [Online]. Available: http://www.isi.edu/ johnh/PAPERS/Syed07a.html

[25] J. Wills, W. Ye, and J. Heidemann, "Low-power Acoustic Modem for Dense Underwater Sensor Networks," in Proceedings of the first ACM international workshop on underwater networks (WUWNet), 2006.

[26] M. Tao, S. Haoshan, and W. Yu, “A MAC Protocol for Underwater Sensor Networks," pp. 144-148, 2007.

[27] R. K. Creber, J. A. Rice, P. A. Baxley, and C. Fletcher, "Performance of undersea acoustic networking using RTS/CTS handshaking and ARQ retransmission," in Proceedings of the IEEE Oceans conference, 2001, pp. 2083-2086. 
[28] F. Salva-Garau and M. Stojanovic, "Multi-cluster protocol for ad hoc underwater acoustic networks," in Proceedings of the IEEE Oceans Conference, September 2003, pp. 91-98.

[29] D. Pompili, T. Melodia, and I. F. Akyildiz, "A Distributed CDMA Medium Access Control for Underwater Acoustic Sensor Networks," in Submitted for Publication, August 2006.

[30] H. Doukkali, L. Nuaymi, and S. Houcke, "Distributed MAC protocols for Underwater Acoustic Data Networks," Vehicular Technology Conference, 2006. VTC-2006 Fall 2006 IEEE 64th, pp. 1-5, 2006.

[31] P. Xie and J.-H. Cui, "R-MAC: An Energy-Efficient MAC Protocol for Underwater Sensor Networks," in Proceedings of the international conference on wireless algorithms, systems, and applications (WASA'07), 2007.

[32] V. Rodoplu and M. Park, "An energy-efficient MAC protocol for underwater wireless acoustic networks," in Proceedings of the MTS/IEEE, Oceans conference, 2005, 2005, pp. 1198-1203.

[33] J. M. Morris, "Optimal Blocklengths for ARQ Error Control Schemes," IEEE Transactions on Communications, vol. COM-27, No. 2, pp. 488-493, 1979.

[34] A. S. Tanenbaum, Computer Networks, 3rd ed. Prentice Hall, 1996.

[35] M. Stojanovic, "Optimization of a Data Link Protocol for an Underwater Acoustic Channel," in Proceedings of the IEEE Oceans conference, 2005, pp. 68-73.

[36] P. F. Turney, "An improved Stop-and-Wait ARQ Logic for Data Transmission in Mobile Radio Systems," IEEE Transactions on Communications, vol. COM-29, No. 1, pp. 68-71, 1981.

[37] H.-P. Tan, W. K. Seah, , and L. Doyle, “A Multi-hop ARQ Protocol for Underwater Acoustic Networks," in Proceedings of the IEEE Oceans conference, 2007. 
[38] P. Xie and J.-H. Cui, "An FEC-based Reliable Data Transport Protocol for Underwater Sensor Networks," in Proceedings of the 16th international conference, 2007, pp. 747-753.

[39] M. Luby, M. Mitzenmacher, A. Shokrollahi, D. Spielman, and V. Stemann, "Practical Loss-Resilient Codes," in Proceedings of the ACM conference, 1997, pp. 150-159.

[40] H.-P. Tan, W. Seah, and L. Doyle, "A Multi-hop ARQ Protocol for an Underwater Acoustic Channel," in Proceedings of the IEEE Oceans Conference, 2007.

[41] Z. Guo, B. Wang, and J.-H. Cui, "Efficient error recovery using network coding in underwater sensor networks," in Proceedings of the IFIP networking conference, 2007, pp. 227-238.

[42] Z. Guo, "On applying network coding to underwater sensor networks," in Proceedings of the ACM WUWNET conference, 2006.

[43] V. Bharghavan, A. Demers, S. Shenker, , and L. Zhang, "MACAW: A Media Access Protocol for Wireless LAN's," in Proceedings of the conference on communications architectures, protocols and applications, 1994, pp. 212-225.

[44] P. Karn, "MACA - a new channel access method for packet radio," in ARRL/CRRL Amateur Radio 9th Computer Networking Conference, September 1990, pp. 134140.

[45] M. Chitre, J. Potter, and O. S. Heng, "Underwater acoustic channel characterization for medium-range shallow water communications," in Proceedings of the IEEE Oceans conference, November 2004, pp. 40-45.

[46] G. Bianchi, "Performance Analysis of the IEEE 802.11 Distributed Coordination Function," IEEE Journal on Selected Areas in Telecommunications, Wireless series, vol. 18, No. 3, pp. 535-547, 2000.

[47] J. Yin, "The Analysis of Performance of IEEE 802.11 MAC Protocol Using Markov Chain," IJCSNS International Journal of Computer Science and Network Security, vol. 7, No. 12, pp. 27-37, 2007. 
[48] Q. Ni, T. Li, T. Turletti, and Y. Xiao, "Saturation throughput analysis of error-prone 802.11 wireless networks," Wiley Journal of Wireless Communications and Mobile Computing (JWCMC), vol. 5, No. 8, pp. 945-956, December 2005.

[49] M. Schwartz, Telecommunication Networks: Protocols, Modeling and Analysis. Addison Wesley, 1987.

[50] H. S. Wang and N. Moayeri, "Finite-State Markov Channels-A Useful Model for Radio Communications Channels," IEEE Transactions on Communications, vol. 43, pp. 163-171, February 1995.

[51] W. Turin and R. V. Nobelen, "Hidden Markov Modeling of Flat Fading Channels," IEEE Journal on Selected Areas in Communications, vol. 16, No. 9, pp. 1809-1817, December 1998.

[52] L. R. Rabiner and B. H. Juang, “An Introduction to Hidden Markov Models," IEEE ASSP Magazine, pp. 4-16, 1986.

[53] L. E. Baum, T. Petrie, G. Soules, and N. Weiss, "A Maximization Technique Occurring in the Statistical Analysis of Probabilistic Functions of Markov Chains," The Annals of Mathematical Statistics, vol. 41, pp. 164-171, February 1970. 
Appendices 


\section{Appendix A: The Stationary Distribution of the Markov Chain}

The stationary distribution of the Markov chain for a backoff stage 0 and a backoff counter 0 for $2 \mathrm{MAC}$ is calculated as follows:

$$
\begin{aligned}
& 1=\frac{\pi_{0,0}}{2}\left[\sum_{i=0}^{m-1} p^{i}\left(\frac{5}{4}\right)^{i} \cdot W+\frac{p^{m}}{(1-p)}\left(\frac{5}{4}\right)^{m} \cdot W+\frac{1}{(1-p)}\right]= \\
& =\frac{\pi_{0,0}}{2}\left[W\left(\sum_{i=0}^{m-1}\left(\frac{5}{4} p\right)^{i}+\frac{\left(\frac{5}{4} p\right)^{m}}{(1-p)}\right)+\frac{1}{(1-p)}\right]= \\
& =\frac{\pi_{0,0}}{2}\left[W\left(\frac{1-\left(\frac{5}{4} p\right)^{m}}{1-\left(\frac{5}{4} p\right)}+\frac{\left(\frac{5}{4} p\right)^{m}}{(1-p)}\right)+\frac{1}{(1-p)}\right]= \\
& =\frac{\pi_{0,0}}{2}\left[W\left(\frac{1-p-\left(\frac{5}{4} p\right)^{m}+p\left(\frac{5}{4} p\right)^{m}+\left(\frac{5}{4} p\right)^{m}-\left(\frac{5}{4} p\right)\left(\frac{5}{4} p\right)^{m}}{\left(1-\left(\frac{5}{4} p\right)\right)(1-p)}\right)+\frac{1}{(1-p)}\right]= \\
& =\frac{\pi_{0,0}}{2}\left[W\left(\frac{1-p-\left(\frac{1}{4} p\right)\left(\frac{5}{4} p\right)^{m}}{\left(1-\left(\frac{5}{4} p\right)\right)(1-p)}\right)+\frac{1}{(1-p)}\right]= \\
& =\frac{\pi_{0,0}}{2}\left[\frac{W-W \cdot p-W \cdot\left(\frac{1}{4} p\right)\left(\frac{5}{4} p\right)^{m}}{\left(1-\left(\frac{5}{4} p\right)\right)(1-p)}+\frac{1}{(1-p)}\right]= \\
& =\frac{\pi_{0,0}}{2}\left[\frac{W-W \cdot p-W \cdot\left(\frac{1}{4} p\right)\left(\frac{5}{4} p\right)^{m}+1-\left(\frac{5}{4} p\right)}{\left(1-\left(\frac{5}{4} p\right)\right)(1-p)}\right]= \\
& =\frac{\pi_{0,0}}{2}\left[\frac{W-\left(\frac{5}{4}-\frac{1}{4}\right) W \cdot p-W \cdot\left(\frac{1}{4} p\right)\left(\frac{5}{4} p\right)^{m}+1-\left(\frac{5}{4} p\right)}{\left(1-\left(\frac{5}{4} p\right)\right)(1-p)}\right]= \\
& =\frac{\pi_{0,0}}{2}\left[\frac{W-\left(\frac{5}{4} p\right) \cdot W+\left(\frac{1}{4} p\right) \cdot W-W \cdot\left(\frac{1}{4} p\right)\left(\frac{5}{4} p\right)^{m}+1-\left(\frac{5}{4} p\right)}{\left(1-\left(\frac{5}{4} p\right)\right)(1-p)}\right]= \\
& =\frac{\pi_{0,0}}{2}\left[\frac{W\left(1-\frac{5}{4} p\right)+\left(\frac{1}{4} p\right) \cdot W\left(1-\left(\frac{5}{4} p\right)^{m}\right)+1-\left(\frac{5}{4} p\right)}{\left(1-\left(\frac{5}{4} p\right)\right)(1-p)}\right]= \\
& =\frac{\pi_{0,0}}{2}\left[\frac{(W+1)\left(1-\left(\frac{5}{4} p\right)\right)+\left(\frac{1}{4} p\right) W\left(1-\left(\frac{5}{4} p\right)^{m}\right)}{\left(1-\left(\frac{5}{4} p\right)\right)(1-p)}\right]
\end{aligned}
$$




\section{Appendix A: (continued)}

Then, the stationary distribution is:

$$
\pi_{0,0}=\frac{8 \cdot\left(1-\left(\frac{5}{4} p\right)\right)(1-p)}{4 \cdot(W+1)\left(1-\left(\frac{5}{4} p\right)\right)+W p\left(1-\left(\frac{5}{4} p\right)^{m}\right)}
$$




\section{Appendix B: The Throughput Efficiency of SW-MER}

The throughput efficiency of SW-MER is calculated as follows:

$$
\begin{aligned}
& \eta=\frac{T_{d}}{T_{f}} \\
& \Rightarrow \eta=\frac{w \frac{D_{d}}{r}}{\left[\sum_{k=0}^{\infty}\left(4^{k-1} \cdot P^{\left(2^{k-1}-1\right)} \cdot(1-P)\right)\right]\left[T_{t x d}+T_{t x a}+2\left(T_{s w}+T_{\text {prop }}\right)\right]}= \\
& =\frac{w \frac{D_{d}}{r}}{(1-P)\left[\sum_{k=0}^{\infty}\left(4^{k-1} \cdot P^{\left(2^{k-1}-1\right)}\right)\right]\left[T_{t x d}+T_{t x a}+2\left(T_{s w}+T_{\text {prop }}\right)\right]}= \\
& =\frac{D_{d}}{(1-P)\left[\sum_{k=0}^{\infty}\left(4^{k-1} \cdot P^{\left(2^{k-1}-1\right)}\right)\right] \frac{r}{w}\left[T_{t x d}+T_{t x a}+2\left(T_{s w}+T_{p r o p}\right)\right]}= \\
& =\frac{D_{d}}{(1-P)\left[\sum_{k=0}^{\infty}\left(4^{k-1} \cdot P^{\left(2^{k-1}-1\right)}\right)\right] \frac{r}{w}\left[w \frac{D_{f}}{r}+\frac{D_{a c k}}{r}+2\left(T_{s w}+T_{p r o p}\right)\right]}= \\
& =\frac{D_{d}}{(1-P)\left[\sum_{k=0}^{\infty}\left(4^{k-1} \cdot P^{\left(2^{k-1}-1\right)}\right)\right]\left[D_{f}+\frac{D_{a c k}}{w}+2 \frac{r}{w}\left(T_{s w}+T_{\text {prop }}\right)\right]}= \\
& =\frac{D_{d}}{(1-P)\left[\sum_{k=0}^{\infty}\left(4^{k-1} \cdot P^{\left(2^{k-1}-1\right)}\right)\right]\left[D_{d}+D_{o h}+\frac{D_{a c k}}{w}+2 \frac{r}{w}\left(T_{s w}+T_{p r o p}\right)\right]}= \\
& =\frac{D_{d}}{(1-P)\left[\sum_{k=0}^{\infty}\left(4^{k-1} \cdot P^{\left(2^{k-1}-1\right)}\right)\right]\left[D_{d}+D_{o h}+\frac{D_{a c k}}{w}+2 \frac{r}{w}\left(T_{s w}+T_{p r o p}\right)\right]}
\end{aligned}
$$




\section{Appendix B: (continued)}

$$
\Rightarrow \eta=\frac{D_{d}}{[1-B E R]^{D_{d}+D_{o h}}\left[\sum_{k=0}^{\infty}\left(4^{k-1} \cdot\left(1-(1-B E R)^{D_{d}+D_{o h}}\right)^{\left(2^{(k-1)-1}\right)}\right)\right]\left[D_{d}+e\right]}
$$

in which $e$ is,

$$
e=\frac{D_{a c k}}{w}+\frac{2 \cdot r\left(T_{s w}+T_{\text {prop }}\right)}{w}+D_{o h}
$$




\begin{abstract}
About the Author
Mr. Daladier Jabba is a Ph.D. candidate in the Department of Computer Science and Engineering at the University of South Florida. Mr. Jabba received the Bachelor of Science degree in August 1991 from the Department of System Engineering, Universidad del Norte, Barranquilla, Colombia. He received his M.S. degree in Computer Science from ITESM (Mexico) and UNAB (Colombia) in 2002. He also received his M.S. degree in Computer Engineering from USF (US) in 2007. He is a COLCIENCIAS - LASPAU scholar at Universidad del Norte, Barranquilla, Colombia. His research interest focuses on underwater acoustic and wireless networks.
\end{abstract}

